\title{
SPECIAL VALUES FOR CONFORMALLY INVARIANT SYSTEMS ASSOCIATED TO MAXIMAL PARABOLICS OF QUASI-HEISENBERG TYPE
}

\author{
TOSHIHISA KUBO
}

\begin{abstract}
In this paper we construct conformally invariant systems of first order and second order differential operators associated to a homogeneous line bundle $\mathcal{L}_{s} \rightarrow G_{0} / Q_{0}$ with $Q_{0}$ a maximal parabolic subgroup of quasiHeisenberg type. This generalizes the results by Barchini, Kable, and Zierau. To do so we use techniques different from ones used by them.
\end{abstract}

\section{INTRODUCTION}

The main work of this paper concerns systems of differential operators that are equivariant under an action of a Lie algebra. We call such systems conformally invariant. To explain the meaning of the equivariance condition, suppose that $\mathcal{V} \rightarrow M$ is a vector bundle over a smooth manifold $M$ and $\mathfrak{g}$ is a Lie algebra of first order differential operators that act on sections of $\mathcal{V}$. A linearly independent list $D_{1}, \ldots, D_{n}$ of linear differential operators on sections of $\mathcal{V}$ is called a conformally invariant system if, for each $X \in \mathfrak{g}$, there are smooth functions $C_{i j}^{X}(m)$ on $M$ so that, for all $1 \leq i \leq n$, and sections $f$ of $\mathcal{V}$, we have

$$
\left(\left[X, D_{i}\right] \bullet f\right)(m)=\sum_{j=1}^{n} C_{j i}^{X}(m)\left(D_{j} \bullet f\right)(m),
$$

where $\left[X, D_{j}\right]=X D_{j}-D_{j} X$. (See Definition 2.3 for the precise definition.) Here, the dot $\bullet$ denotes the action of differential operators on smooth functions.

An important consequence of the definition (1.1) is that the common kernel of the operators in the conformally invariant system $D_{1}, \ldots, D_{n}$ is invariant under a Lie algebra action. The representation theoretic question of understanding the common kernel as a $\mathfrak{g}$-module is an open question (except for a small number of very special examples).

The notion of conformally invariant systems generalizes that of quasi-invariant differential operators introduced by Kostant in [19] and is related to work of Huang $([9])$. It is also compatible with the definition given by Ehrenpreis in [7. Conformally invariant systems are explicitly or implicitly presented in the work of Davidson-Enright-Stanke ([4]), Kobayashi-Orsted ([18]), and Wallach ([25]), among others. They are also related to the project of Dobrev on constructing intertwining differential operators of arbitrary order. (See for example [5] and [6].) Much

Received by the editors September 9, 2012.

2010 Mathematics Subject Classification. Primary 22E46; Secondary 17B10, 22E47.

Key words and phrases. Conformally invariant systems, intertwining differential operator, real flag manifold. 
of the published work is for the case that $M=G / Q$ with $Q=L N, N$ abelian. The systematic study of conformally invariant systems started with the work of Barchini-Kable-Zierau in [1] and [2] and is continued in [11 15], and 21 23].

Although the theory of conformally invariant systems can be viewed as a geometric-analytic theory, it is closely related to algebraic objects such as generalized Verma modules. It has been shown in 2 that a conformally invariant system yields a homomorphism between certain generalized Verma modules. The classification of non-standard homomorphisms between generalized Verma modules is an open problem. In 22 and [23, it is determined whether or not the homomorphisms between the generalized Verma modules that arise from certain conformally invariant systems are standard.

The main goal of this paper is to build systems of differential operators that satisfy condition (1.1) when $M$ is a homogeneous manifold $G / Q$ with $Q$ in a certain class of maximal parabolic subgroups. This is to construct systems $D_{1}, \ldots, D_{n}$ acting on sections of bundles $\mathcal{V}_{s} \rightarrow G / Q$ over $G / Q$ in a systematic manner and to determine the bundles $\mathcal{V}_{s}$ on which the systems are conformally invariant. The method that we use is different from one used by Barchini-Kable-Zierau in [1].

To describe our work more precisely, let $G$ be a complex, simple, connected, simply-connected Lie group with Lie algebra $\mathfrak{g}$. It is known that $\mathfrak{g}$ has a $\mathbb{Z}$-grading $\mathfrak{g}=\bigoplus_{j=-r}^{r} \mathfrak{g}(j)$ so that $\mathfrak{q}=\mathfrak{g}(0) \oplus \bigoplus_{j>0} \mathfrak{g}(j)=\mathfrak{l} \oplus \mathfrak{n}$ is a parabolic subalgebra of $\mathfrak{g}$. Let $Q=N_{G}(\mathfrak{q})=L N$. For a real form $\mathfrak{g}_{0}$ of $\mathfrak{g}$, define $G_{0}$ to be an analytic subgroup of $G$ with Lie algebra $\mathfrak{g}$. Set $Q_{0}=N_{G_{0}}(\mathfrak{q})$. Our manifold is $M=G_{0} / Q_{0}$ and we consider a line bundle $\mathcal{L}_{s} \rightarrow G_{0} / Q_{0}$ for each $s \in \mathbb{C}$. By the Bruhat theory, $G_{0} / Q_{0}$ admits an open dense submanifold $\bar{N}_{0} Q_{0} / Q_{0}$. We restrict our bundle to this submanifold. The systems that we study are on smooth sections of the restricted bundle.

To build systems of differential operators we observe that $L$ acts by the adjoint representation on $\mathfrak{g}(1)$ with a unique open orbit. This makes $\mathfrak{g}(1)$ a prehomogeneous vector space. Our construction is based on the invariant theory of a prehomogeneous vector space. It is natural to associate $L$-equivariant polynomial maps, called covariant maps, to the prehomogeneous vector space $(L, \mathrm{Ad}, \mathfrak{g}(1))$. To define our systems of differential operators, we use covariant maps $\tau_{k}: \mathfrak{g}(1) \rightarrow \mathfrak{g}(-r+k) \otimes \mathfrak{g}(r)$ that are associated to $\mathfrak{g}(1)$. (See Definition 3.1.) Each $\tau_{k}$ can be thought of as giving the symbols of the differential operators that we study.

Let $\mathfrak{g}(-r+k) \otimes \mathfrak{g}(r)=V_{1} \oplus \cdots \oplus V_{m}$ be the irreducible decomposition of $\mathfrak{g}(-r+$ $k) \otimes \mathfrak{g}(r)$ as an $L$-module. The covariant map $\tau_{k}$ induces an $L$-equivariant linear map $\left.\tilde{\tau}_{k}\right|_{V_{j}^{*}}: V_{j}^{*} \rightarrow \mathcal{P}^{k}(\mathfrak{g}(1))$ with $V_{j}^{*}$ the dual of an irreducible constituent $V_{j}$ of $\mathfrak{g}(-r+k) \otimes \mathfrak{g}(r)$ and $\mathcal{P}^{k}(\mathfrak{g}(1))$ the space of homogeneous polynomials on $\mathfrak{g}(1)$ of degree $k$. We define differential operators from $\left.\tilde{\tau}_{k}\right|_{V_{j}^{*}}\left(Y^{*}\right)$. For $Y^{*} \in V_{j}^{*}$, let $\Omega_{k}\left(Y^{*}\right)$ denote the $k$-th order differential operators that are constructed from $\left.\tilde{\tau}_{k}\right|_{V_{j}^{*}}\left(Y^{*}\right)$. We say that a list of differential operators $D_{1}, \ldots, D_{n}$ is the $\left.\Omega_{k}\right|_{V_{j}^{*}}$ system if it is equivalent (see Definition 2.4) to a list of differential operators $\Omega_{k}\left(Y_{1}^{*}\right), \ldots, \Omega_{k}\left(Y_{n}^{*}\right)$, where $\left\{Y_{1}^{*}, \ldots, Y_{n}^{*}\right\}$ is a basis for $V_{j}^{*}$. By construction the $\left.\Omega_{k}\right|_{V_{j}^{*}}$ system consists of $\operatorname{dim}_{\mathbb{C}}\left(V_{j}\right)$ operators.

The conformal invariance of the $\left.\Omega_{k}\right|_{V_{j}^{*}}$ system depends on the complex parameter $s$ for the line bundle $\mathcal{L}_{s}$. Then we say that the $\left.\Omega_{k}\right|_{V_{j}^{*}}$ system has special value $s_{0}$ if the system is conformally invariant on the line bundle $\mathcal{L}_{s_{0}}$. The special values for 
the case that $\operatorname{dim}([\mathfrak{n}, \mathfrak{n}])=1$ for $\mathfrak{q}=\mathfrak{l} \oplus \mathfrak{n}$ are studied by Barchini-Kable-Zierau in [1] and [2], and the author in 21] and 24].

In this paper we consider a more general case; namely, $\mathfrak{q}=\mathfrak{l} \oplus \mathfrak{n}$ is a maximal parabolic subalgebra and $\mathfrak{n}$ satisfies the condition that $[\mathfrak{n},[\mathfrak{n}, \mathfrak{n}]]=0$ and $\operatorname{dim}_{\mathbb{C}}([\mathfrak{n}, \mathfrak{n}])>1$. We call such maximal parabolic subalgebras quasi-Heisenberg type. In this case we have $r=2$ in the $\mathbb{Z}$-grading $\mathfrak{g}=\bigoplus_{j=-r}^{r} \mathfrak{g}(j)$. Therefore the $\Omega_{k}$ systems for $k \geq 5$ are zero. We determine the special values of the $\Omega_{1}$ system and $\Omega_{2}$ systems associated to the parabolic subalgebras under consideration. Here is one remark on the special values for the $\Omega_{2}$ systems. In contrast to the fact that the special value for the $\Omega_{1}$ system is determined completely, we leave three cases open for $\Omega_{2}$ systems, namely, the type $1 \mathrm{~b}$ case for $\mathfrak{q}$ of type $B_{n}(n-1)$, the type 3 case for $\mathfrak{q}$ of type $C_{n}(i)$, and the case when $\mathfrak{q}$ is of type $D_{n}(n-2)$. (See Section 4.3 and Definition 6.20 for the definitions of the types of $\mathfrak{q}$, and type $1 b$ and type 3 cases, respectively.) This is because we could not handle these cases uniformly. We closed these cases in [22].

Now we make a short remark about finding special values for $\Omega_{2}$ systems. Although the special value of $s$ for the $\Omega_{1}$ system is easily found by computing the bracket $\left[X, \Omega_{1}\left(Y_{i}^{*}\right)\right]$, it is not easy to find the special values for $\Omega_{2}$ systems by a direct computation. (See Section 5 of [1].) In this paper, to find the special values for the $\Omega_{2}$ systems, we use two reduction techniques. These techniques significantly reduce the amount of computations. (See Proposition 2.16 and Proposition 7.13.)

This paper consists of seven sections, including this introduction, and one appendix. We now outline the contents of the rest of this paper. In Section 2, we first recall the definition of conformally invariant systems of differential operators and then collect some useful formulas. In Section 3 , the construction of the $\Omega_{k}$ systems is given precisely. To construct the $\Omega_{1}$ system and $\Omega_{2}$ systems for maximal parabolic subalgebra $\mathfrak{q}$ of quasi-Heisenberg type, we study such a parabolic subalgebra $\mathfrak{q}$ and the associated 2-grading $\mathfrak{g}=\bigoplus_{j=-2}^{2} \mathfrak{g}(j)$ in Section 4 .

We construct the $\Omega_{1}$ system and find its special value in Section 5 . In this section we also fix normalizations for root vectors. The normalizations play an important role in constructing the system. We show that the special value $s_{1}$ for the $\Omega_{1}$ system is $s_{1}=0$. This is done in Theorem 5.7 .

To build the $\Omega_{2}$ systems, we need to find the irreducible constituents $V^{*}$ of $\mathfrak{l}^{*} \otimes \mathfrak{z}(\mathfrak{n})^{*}$ so that $\left.\tilde{\tau}_{2}\right|_{V^{*}} \neq 0$. In Section [6] we show preliminary results to find such irreducible constituents. First we decompose $\mathfrak{l} \otimes \mathfrak{z}(\mathfrak{n})$ into the direct sum of the irreducible constituents. By using the decomposition results, we then determine the candidates of the irreducible constituents $V^{*}$ so that $\left.\tilde{\tau}_{2}\right|_{V^{*}} \neq 0$. We build the $\Omega_{2}$ systems and find their special values in Section 7 . The special values are determined in Theorem 7.16 .

Finally, in Appendix A we summarize the miscellaneous useful data for the parabolic subalgebras under consideration.

\section{Conformally invariant Systems}

In this section we recall from [2] the definition of a conformally invariant system of differential operators. We also collect some properties of such a system of differential operators.

2.1. Conformally invariant systems. Let $\mathfrak{g}_{0}$ be a real Lie algebra and $\mathcal{X}(M)$ be the space of smooth vector fields on a smooth manifold $M$. 
Definition 2.1 ([2] page 790]). A smooth manifold $M$ is called a $\mathfrak{g}_{0}$-manifold if there is an $\mathbb{R}$-linear map $\pi_{M}: \mathfrak{g}_{0} \rightarrow C^{\infty}(M) \oplus X(M)$ so that for all $X, Y \in \mathfrak{g}_{0}$,

$$
\pi_{M}([X, Y])=\left[\pi_{M}(X), \pi_{M}(Y)\right] .
$$

For each $X \in \mathfrak{g}_{0}$, we write $\pi_{M}(X)=\pi_{0}(X)+\pi_{1}(X)$ with $\pi_{0}(X) \in C^{\infty}(M)$ and $\pi_{1}(X) \in X(M)$. We denote by $\mathbb{D}(\mathcal{V})$ the space of differential operators on smooth sections of $\mathcal{V}$.

Definition 2.2 ([2, page 791]). Let $M$ be a $\mathfrak{g}_{0}$-manifold. A vector bundle $\mathcal{V} \rightarrow M$ is called a $\mathfrak{g}_{0}$-bundle if there is an $\mathbb{R}$-linear map $\pi_{v}: \mathfrak{g}_{0} \rightarrow \mathbb{D}(\mathcal{V})$ that satisfies the following properties:

(B1) We have $\pi_{\mathcal{V}}([X, Y])=\left[\pi_{\mathcal{V}}(X), \pi_{\mathcal{V}}(Y)\right]$ for all $X, Y \in \mathfrak{g}_{0}$.

(B2) In $\mathbb{D}(\mathcal{V}),\left[\pi_{\mathcal{V}}(X), f\right]=\pi_{1}(X) \bullet f$ for all $X \in \mathfrak{g}_{0}$ and $f \in C^{\infty}(M)$.

Definition 2.3 ([2, page 791]). Let $\mathcal{V} \rightarrow M$ be a $\mathfrak{g}_{0}$-bundle. A conformally invariant system on $\mathcal{V}$ with respect to $\pi_{v}$ is a list of differential operators $D_{1}, \ldots, D_{m}$ $\in \mathbb{D}(\mathcal{V})$ so that the following two conditions hold:

(S1) At each point $p \in M$, the list $D_{1}, \ldots, D_{m}$ is linearly independent.

(S2) For each $X \in \mathfrak{g}_{0}$, there is a matrix $C(X)$ in $M_{m \times m}\left(C^{\infty}(M)\right)$ so that in $\mathbb{D}(\mathcal{V})$

$$
\left[\pi_{\mathcal{V}}(X), D_{i}\right]=\sum_{j=1}^{m} C_{j i}(X) D_{j} .
$$

The map $C: \mathfrak{g}_{0} \rightarrow M_{m \times m}\left(C^{\infty}(M)\right)$ is called the structure operator of the conformally invariant system.

If $\mathfrak{g}$ is the complexification of $\mathfrak{g}_{0}$, then $\mathfrak{g}$-manifolds and $\mathfrak{g}$-bundles are defined by extending the $\mathfrak{g}_{0}$-action $\mathbb{C}$-linearly. On p. 792 of [2], the equivalence of two conformally invariant systems are defined. For later convenience we apply the same definition to any system of differential operators. (See Definition 3.5.)

Definition 2.4. We say that two systems of differential operators (not necessarily conformally invariant) $D_{1}, \ldots, D_{n}$ and $D_{1}^{\prime}, \ldots, D_{n}^{\prime}$ in $\mathbb{D}(\mathcal{V})$ are equivalent if there is a matrix $A \in G L\left(n, C^{\infty}(M)\right)$ so that, for $1 \leq i \leq n$,

$$
D_{i}^{\prime}=\sum_{j=1}^{n} A_{j i} D_{j} .
$$

Definition 2.5 ([2, page 793]). A conformally invariant system $D_{1}, \ldots, D_{n}$ is called reducible if there are an equivalent conformally invariant system $D_{1}^{\prime}, \ldots, D_{n}^{\prime}$ and an $m<n$ such that the system $D_{1}^{\prime}, \ldots, D_{m}^{\prime}$ is conformally invariant. Otherwise we say that $D_{1}, \ldots, D_{n}$ is irreducible.

We now specialize the $\mathfrak{g}$-manifold and $\mathfrak{g}$-bundle that we will work with. Let $G$ be a complex, simple, connected, simply-connected Lie group with Lie algebra $\mathfrak{g}$. Such $G$ contains a maximal connected solvable subgroup $B$. Write $\mathfrak{b}=\mathfrak{h} \oplus \mathfrak{u}$ for its Lie algebra with $\mathfrak{h}$ the Cartan subalgebra and $\mathfrak{u}$ the nilpotent subalgebra. Let $\mathfrak{q} \supset \mathfrak{b}$ be a parabolic subalgebra of $\mathfrak{g}$. We define $Q=N_{G}(\mathfrak{q})$, a parabolic subgroup of $G$. Write $Q=L N$ for the Levi decomposition of $Q$, with $L$ the Levi subgroup and $N$ the nilpotent subgroup.

Let $\mathfrak{g}_{0}$ be a real form of $\mathfrak{g}$ in which the complex parabolic subalgebra $\mathfrak{q}$ has a real form $\mathfrak{q}_{0}$, and let $G_{0}$ be the analytic subgroup of $G$ with Lie algebra $\mathfrak{g}_{0}$. Define 
$Q_{0}=N_{G_{0}}(\mathfrak{q}) \subset Q$ and write $Q_{0}=L_{0} N_{0}$. We will work with $M=G_{0} / Q_{0}$ for a class of a maximal parabolic subgroup $Q_{0}$ that will be specified in Section 4

Next, we need to specify a vector bundle $\mathcal{V}$ on $M$. To this end let $\Delta=\Delta(\mathfrak{g}, \mathfrak{h})$ be the set of roots of $\mathfrak{g}$ with respect to $\mathfrak{h}$. Let $\Delta^{+}$be the positive system attached to $\mathfrak{b}$ and denote by $\Pi$ the set of simple roots. For each subset $S \subset \Pi$, let $\mathfrak{q}_{S}$ be the corresponding standard parabolic subalgebra. Write $\mathfrak{q}_{S}=\mathfrak{l}_{S} \oplus \mathfrak{n}_{S}$ with Levi factor $\mathfrak{l}_{S}=\mathfrak{h} \oplus \bigoplus_{\alpha \in \Delta_{S}} \mathfrak{g}_{\alpha}$ and nilpotent radical $\mathfrak{n}_{S}=\bigoplus_{\alpha \in \Delta^{+} \backslash \Delta_{S}} \mathfrak{g}_{\alpha}$, where $\Delta_{S}=\{\alpha \in \Delta \mid \alpha \in \operatorname{span}(\Pi \backslash S)\}$. If $Q_{0}$ is a maximal parabolic, then there exists a unique simple root $\alpha_{\mathfrak{q}} \in \Pi$ so that $\mathfrak{q}=\mathfrak{q}_{\left\{\alpha_{\mathfrak{q}}\right\}}$. Let $\lambda_{\mathfrak{q}}$ be the fundamental weight of $\alpha_{\mathfrak{q}}$. The weight $\lambda_{\mathfrak{q}}$ is orthogonal to any roots $\alpha$ with $\mathfrak{g}_{\alpha} \subset[\mathfrak{l}, \mathfrak{l}]$. Hence it exponentiates to a character $\chi_{\mathfrak{q}}$ of $L$. As $\chi_{\mathfrak{q}}$ takes real values on $L_{0}$, for $s \in \mathbb{C}$, the character $\chi^{s}=\left|\chi_{\mathfrak{q}}\right|^{s}$ is well defined on $L_{0}$. Let $\mathbb{C}_{\chi^{s}}$ be the one-dimensional representation of $L_{0}$ with character $\chi^{s}$. The representation $\chi^{s}$ is extended to a representation of $Q_{0}$ by making it trivial on $N_{0}$. Then it deduces a line bundle $\mathcal{L}_{s}$ on $M=G_{0} / Q_{0}$ with fiber $\mathbb{C}_{\chi^{s}}$.

The group $G_{0}$ acts on the space

$$
\begin{aligned}
& C_{\chi}^{\infty}\left(G_{0} / Q_{0}, \mathbb{C}_{\chi^{s}}\right) \\
& =\left\{F \in C^{\infty}\left(G_{0}, \mathbb{C}_{\chi^{s}}\right) \mid F(g q)=\chi^{s}\left(q^{-1}\right) F(g) \text { for all } q \in Q_{0} \text { and } g \in G_{0}\right\}
\end{aligned}
$$

by left translation. The action $\pi_{s}$ of $\mathfrak{g}_{0}$ on $C_{\chi}^{\infty}\left(G_{0} / Q_{0}, \mathbb{C}_{\chi^{s}}\right)$ arising from this action is given by

$$
\left(\pi_{s}(Y) \bullet F\right)(g)=\left.\frac{d}{d t} F(\exp (-t Y) g)\right|_{t=0}
$$

for $Y \in \mathfrak{g}_{0}$. This action is extended $\mathbb{C}$-linearly to $\mathfrak{g}$ and then naturally to the universal enveloping algebra $\mathcal{U}(\mathfrak{g})$. We use the same symbols for the extended actions.

Let $\bar{N}_{0}$ be the unipotent subgroup opposite to $N_{0}$. By the Bruhat theory, the subset $\bar{N}_{0} Q_{0}$ is open and dense in $G_{0}$. Then the restriction map $C_{\chi}^{\infty}\left(G_{0} / Q_{0}, \mathbb{C}_{\chi^{s}}\right) \rightarrow$ $C^{\infty}\left(\bar{N}_{0}, \mathbb{C}_{\chi^{s}}\right)$ is an injection, where $C^{\infty}\left(\bar{N}_{0}, \mathbb{C}_{\chi^{s}}\right)$ is the space of the smooth functions from $\bar{N}_{0}$ to $\mathbb{C}_{\chi^{s}}$. Then, for $u \in \mathcal{U}(\mathfrak{g})$ and $F \in C_{\chi}^{\infty}\left(G_{0} / Q_{0}, \mathbb{C}_{\chi^{s}}\right)$, we let $f=\left.F\right|_{\bar{N}_{0}}$ and define the action of $\mathcal{U}(\mathfrak{g})$ on the image of the restriction map by

$$
\pi_{s}(u) \bullet f=\left.\left(\pi_{s}(u) \bullet F\right)\right|_{\bar{N}_{0}} .
$$

The line bundle $\mathcal{L}_{s} \rightarrow G_{0} / Q_{0}$ restricted to $\bar{N}_{0}$ is the trivial bundle $\bar{N}_{0} \times \mathbb{C}_{\chi^{s}} \rightarrow \bar{N}_{0}$. By slight abuse of notation, we refer to the trivial bundle over $\bar{N}_{0}$ as $\mathcal{L}_{s}$. Then in practice our manifold $M$ will be $M=\bar{N}_{0}$ and our vector bundle will be the trivial bundle.

Now we show that, with the action $\pi_{s}$, the group $\bar{N}_{0}$ and the trivial bundle $\mathcal{L}_{s}$ are a $\mathfrak{g}$-manifold and $\mathfrak{g}$-bundle, respectively. Let $\overline{\mathfrak{n}}$ and $\mathfrak{q}$ be the complexifications of the Lie algebras of $\bar{N}_{0}$ and $Q_{0}$, respectively; we have the direct sum $\mathfrak{g}=\overline{\mathfrak{n}} \oplus \mathfrak{q}$. For $Y \in \mathfrak{g}$, write $Y=Y_{\overline{\mathfrak{n}}}+Y_{\mathfrak{q}}$ for the decomposition of $Y$ in this direct sum. Similarly, write the Bruhat decomposition of $g \in \bar{N}_{0} Q_{0}$ as $g=\overline{\mathbf{n}}(g) \mathbf{q}(g)$ with $\overline{\mathbf{n}}(g) \in \bar{N}_{0}$ and $\mathbf{q}(g) \in Q_{0}$. For $Y \in \mathfrak{g}_{0}$, we have

$$
Y_{\overline{\mathfrak{n}}}=\left.\frac{d}{d t} \overline{\mathbf{n}}(\exp (t Y))\right|_{t=0},
$$


and a similar equality holds for $Y_{\mathfrak{q}}$. Define a right action $R$ of $\mathcal{U}(\overline{\mathfrak{n}})$ on $C^{\infty}\left(\bar{N}_{0}, \mathbb{C}_{\chi^{s}}\right)$ by

$$
(R(X) \bullet f)(\bar{n})=\left.\frac{d}{d t} f(\bar{n} \exp (t X))\right|_{t=0}
$$

for $X \in \overline{\mathfrak{n}}_{0}$ and $f \in C^{\infty}\left(\bar{N}_{0}, \mathbb{C}_{\chi^{s}}\right)$. Observe that, by definition, the differential $d \chi$ of $\chi$ is $d \chi=\lambda_{\mathfrak{q}}$. A direct computation then shows that, for $Y \in \mathfrak{g}$ and $f$ in the image of the restriction map $C_{\chi}^{\infty}\left(G_{0} / Q_{0}, \mathbb{C}_{\chi^{s}}\right) \rightarrow C^{\infty}\left(\bar{N}_{0}, \mathbb{C}_{\chi^{s}}\right)$, we have

$$
\left(\pi_{s}(Y) \bullet f\right)(\bar{n})=s \lambda_{\mathfrak{q}}\left(\left(\operatorname{Ad}\left(\bar{n}^{-1}\right) Y\right)_{\mathfrak{q}}\right) f(\bar{n})-\left(R\left(\left(\operatorname{Ad}\left(\bar{n}^{-1}\right) Y\right)_{\overline{\mathfrak{n}}}\right) \bullet f\right)(\bar{n}) .
$$

Observe that (2.9) implies that the representation $\pi_{s}$ extends to a representation of $\mathcal{U}(\mathfrak{g})$ on the whole space $C^{\infty}\left(\bar{N}_{0}, \mathbb{C}_{\chi^{s}}\right)$. Moreover, it also shows that for all $Y \in \mathfrak{g}$, the linear map $\pi_{s}(Y)$ is in $C^{\infty}\left(\bar{N}_{0}\right) \oplus X\left(\bar{N}_{0}\right)$. Therefore, with this linear map $\pi_{s}$, $\bar{N}_{0}$ is a $\mathfrak{g}$-manifold.

Next, we show that the linear map $\pi_{s}$ gives $\mathcal{L}_{s}$ the structure of a $\mathfrak{g}$-bundle. As $\pi_{s}$ is a representation of $\mathfrak{g}$, condition (B1) of Definition 2.2 is trivial. Thus it suffices to show that condition (B2) holds. Since $\mathcal{L}_{s}$ is the trivial bundle of $\bar{N}_{0}$ with fiber $\mathbb{C}_{\chi^{s}}$, the space of smooth sections of $\mathcal{L}_{s}$ is identified with $C^{\infty}\left(\bar{N}_{0}, \mathbb{C}_{\chi^{s}}\right)$. The following proposition establishes condition (B2) in our situation.

Proposition 2.10. In $\mathbb{D}\left(\mathcal{L}_{s}\right)$, for $Y \in \mathfrak{g}$ and $f \in C^{\infty}\left(\bar{N}_{0}\right)$, we have

$$
\left(\left[\pi_{s}(Y), f\right]\right)(\bar{n})=-\left(R\left(\left(\operatorname{Ad}\left(\bar{n}^{-1}\right) Y\right)_{\bar{n}}\right) \bullet f\right)(\bar{n}) .
$$

Proof. This follows from the definition of $\left[\pi_{s}(Y), f\right]$ and formula (2.9).

2.2. Properties of conformally invariant systems. In Section 3 we are going to construct systems of differential operators on $\mathcal{L}_{s}$. These systems of operators will satisfy several properties of conformally invariant systems. For convenience we collect those properties from [2] here.

We first define an action of $L_{0}$ on $\mathbb{D}\left(\mathcal{L}_{s}\right)$. As on p. 805 of [2], we define an action of $L_{0}$ on $C^{\infty}\left(\bar{N}_{0}, \mathbb{C}_{\chi^{s}}\right)$ by

$$
(l \cdot f)(\bar{n})=\chi^{s}(l) f\left(l^{-1} \bar{n} l\right) .
$$

This action agrees with the action of $L_{0}$ by the left translation on the image of the restriction map $C_{\chi}^{\infty}\left(G_{0} / Q_{0}, \mathbb{C}_{\chi^{s}}\right) \rightarrow C^{\infty}\left(\bar{N}_{0}, \mathbb{C}_{\chi^{s}}\right)$. In terms of this action we define an action of $L_{0}$ on $\mathbb{D}\left(\mathcal{L}_{s}\right)$ by

$$
(l \cdot D) \bullet f=l \cdot\left(D \bullet\left(l^{-1} \cdot f\right)\right) .
$$

Definition 2.12 ([2, page 806]). A conformally invariant system $D_{1}, \ldots, D_{m}$ on $\mathcal{L}_{s} \rightarrow \bar{N}_{0}$ is called $L_{0}$-stable if there is a map $c: L_{0} \rightarrow G L\left(n, C^{\infty}\left(\bar{N}_{0}\right)\right)$ such that

$$
l \cdot D_{i}=\sum_{j=1}^{m} c(l)_{j i} D_{j} .
$$

It is known that there exists a semisimple element $H_{0} \in \mathfrak{l}$ so that $\operatorname{ad}\left(H_{0}\right)$ has only integer eigenvalues on $\mathfrak{g}$ with $\mathfrak{g}(1) \neq\{0\}, \mathfrak{l}=\mathfrak{g}(0), \mathfrak{n}=\bigoplus_{j>0} \mathfrak{g}(j)$, and $\overline{\mathfrak{n}}=$ $\bigoplus_{j>0} \mathfrak{g}(-j)$, where $\mathfrak{g}(j)$ is the $j$-eigenspace of $\operatorname{ad}\left(H_{0}\right)$. (See for example [17, Section X.3].)

Definition 2.13 ([2, page 804]). A conformally invariant system $D_{1}, \ldots, D_{m}$ is called homogeneous if $C\left(H_{0}\right)$ is a scalar matrix, where $C$ is the structure operator of the conformally invariant system. (See Definition 2.3.) 
Proposition 2.14 ([2, Proposition 17]). Any irreducible conformally invariant system is homogeneous.

Define

$$
\mathbb{D}\left(\mathcal{L}_{s}\right)^{\overline{\mathfrak{n}}}=\left\{D \in \mathbb{D}\left(\mathcal{L}_{s}\right) \mid\left[\pi_{s}(X), D\right]=0 \text { for all } X \in \overline{\mathfrak{n}}\right\} .
$$

Observe that in the sense of [2, page 796], the $\mathfrak{g}$-manifold $\bar{N}_{0}$ is straight with respect to the subalgebra $\overline{\mathfrak{n}}$ of $\mathfrak{g}([2$, page 799$])$. Then we state the definition of straight conformally invariant systems specialized to the present situation. (For the general definition see p. 797 of [2].)

Definition 2.15. A conformally invariant system $D_{1}, \ldots, D_{m}$ is said to be straight if $D_{j} \in \mathbb{D}\left(\mathcal{L}_{s}\right)^{\overline{\mathfrak{n}}}$ for $j=1, \ldots, m$.

In general, to show that a given list $D_{1}, \ldots, D_{m}$ of differential operators on $\bar{N}_{0}$ is a conformally invariant system, we need to check (S2) of Definition 2.3 at each point of $\bar{N}_{0}$. Proposition 2.16 below shows that in the case $D_{1}, \ldots, D_{m}$ in $\mathbb{D}\left(\mathcal{L}_{s}\right)^{\overline{\mathfrak{n}}}$, it suffices to check the condition only at the identity $e$.

Proposition 2.16 ([2, Proposition 13]). Let $D_{1}, \ldots, D_{m}$ be a list of operators in $\mathbb{D}\left(\mathcal{L}_{s}\right)^{\overline{\mathfrak{n}}}$. Suppose that the list is linearly independent at $e$ and that there is a map $b: \mathfrak{g} \rightarrow \mathfrak{g l}(m, \mathbb{C})$ such that

$$
\left(\left[\pi_{s}(Y), D_{i}\right] \bullet f\right)(e)=\sum_{j=1}^{m} b(Y)_{j i}\left(D_{j} \bullet f\right)(e)
$$

for all $Y \in \mathfrak{g}, f \in C^{\infty}\left(\bar{N}_{0}, \mathbb{C}_{\chi^{s}}\right)$, and $1 \leq i \leq m$. Then $D_{1}, \ldots, D_{m}$ is a conformally invariant system on $\mathcal{L}_{s}$. The structure operator of the system is given by $C(Y)(\bar{n})=$ $b\left(\operatorname{Ad}\left(\bar{n}^{-1}\right) Y\right)$ for all $\bar{n} \in \bar{N}_{0}$ and $Y \in \mathfrak{g}$.

To end this section we are going to give two formulas that will make certain arguments simple in Section 5 and Section 7.

Proposition 2.17. Let $Y \in \mathfrak{g}$ and $f \in C^{\infty}\left(\bar{N}_{0}, \mathbb{C}_{\chi^{s}}\right)$. For $X, X_{1}, X_{2} \in \overline{\mathfrak{n}}$, we have $\left(\left[\pi_{s}(Y), R(X)\right] \bullet f\right)(\bar{n})=\left(R\left(\left[\left(\operatorname{Ad}\left(\bar{n}^{-1}\right) Y\right)_{\mathfrak{q}}, X\right]_{\overline{\mathfrak{n}}}\right) \bullet f\right)(\bar{n})-s \lambda_{\mathfrak{q}}\left(\left[\operatorname{Ad}\left(\bar{n}^{-1}\right) Y, X\right]_{\mathfrak{q}}\right) f(\bar{n})$ and

$$
\begin{aligned}
([ & \left.\left.\pi_{s}(Y), R\left(X_{1}\right) R\left(X_{2}\right)\right] \bullet f\right)(\bar{n}) \\
= & \left(R\left(\left[\left(\operatorname{Ad}\left(\bar{n}^{-1}\right) Y\right)_{\mathfrak{q}}, X_{1}\right]_{\overline{\mathfrak{n}}}\right) R\left(X_{2}\right) \bullet f\right)(\bar{n})+\left(R\left(X_{1}\right) R\left(\left[\left(\operatorname{Ad}\left(\bar{n}^{-1}\right) Y\right)_{\mathfrak{q}}, X_{2}\right]_{\mathfrak{n}}\right) \bullet f\right)(\bar{n}) \\
& +\left(R\left(\left[\left[\operatorname{Ad}\left(\bar{n}^{-1}\right) Y, X_{1}\right]_{\mathfrak{q}}, X_{2}\right]_{\overline{\mathfrak{n}}}\right) \bullet f\right)(\bar{n})-s \lambda_{\mathfrak{q}}\left(\left[\operatorname{Ad}\left(\bar{n}^{-1}\right) Y, X_{1}\right]_{\mathfrak{q}}\right)\left(R\left(X_{2}\right) \bullet f\right)(\bar{n}) \\
& -s \lambda_{\mathfrak{q}}\left(\left[\operatorname{Ad}\left(\bar{n}^{-1}\right) Y, X_{2}\right]_{\mathfrak{q}}\right)\left(R\left(X_{1}\right) \bullet f\right)(\bar{n})-s \lambda_{\mathfrak{q}}\left(\left[\left[\operatorname{Ad}\left(\bar{n}^{-1}\right) Y, X_{1}\right], X_{2}\right]_{\mathfrak{q}}\right) f(\bar{n}) .
\end{aligned}
$$

Proof. These formulas follow from a direct computation on the left hand side of each equation using (2.9) .

\section{THE $\Omega_{k}$ SYSTEMS}

The purpose of this section is to construct systems of $k$-th order differential operators in $\mathbb{D}\left(\mathcal{L}_{s}\right)^{\overline{\mathfrak{n}}}$ in a systematic manner. We shall call the systems of operators $\Omega_{k}$ systems. 
3.1. Construction of the $\Omega_{k}$ systems. Let $\mathfrak{g}=\bigoplus_{j=-r}^{r} \mathfrak{g}(j)$ on $\mathfrak{g}$ be a $\mathbb{Z}$-grading on $\mathfrak{g}$ with $\mathfrak{g}(1) \neq 0$. By construction, $\mathfrak{q}=\mathfrak{g}(0) \oplus \bigoplus_{j>0} \mathfrak{g}(j)$ is a parabolic subalgebra. Take $L$ to be the analytic subgroup of $G$ with Lie algebra $\mathfrak{g}(0)$. Observe that, by Vinberg's Theorem ([17, Theorem 10.19]), the triple $(L, \mathrm{Ad}, \mathfrak{g}(1))$ is a prehomogeneous vector space, that is, $L$ has an open orbit in $\mathfrak{g}(1)$. To define our systems of differential operators, we use covariant maps ( $L$-equivariant polynomial maps), which we denote by $\tau_{k}$, associated to prehomogeneous vector space $(L, \operatorname{Ad}, \mathfrak{g}(1))$. These maps can be thought of as giving symbols of a class of differential operators that we will study. We would like to acknowledge that the construction of $\tau_{k}$ as in this paper was suggested by Anthony Kable.

Definition 3.1. Let $\mathfrak{g}=\bigoplus_{j=-r}^{r} \mathfrak{g}(j)$ be a graded complex simple Lie algebra with $\mathfrak{g}(1) \neq 0$. Then, for $1 \leq k \leq 2 r$, the map $\tau_{k}$ on $\mathfrak{g}(1)$ is defined by

$$
\begin{aligned}
\tau_{k}: \mathfrak{g}(1) & \rightarrow \mathfrak{g}(-r+k) \otimes \mathfrak{g}(r) \\
X & \mapsto \frac{1}{k !}\left(\operatorname{ad}(X)^{k} \otimes \operatorname{Id}\right) \omega
\end{aligned}
$$

with

$$
\omega=\sum_{\gamma_{j} \in \Delta(\mathfrak{g}(r))} X_{\gamma_{j}}^{*} \otimes X_{\gamma_{j}} \in \mathfrak{g}(-r) \otimes \mathfrak{g}(r)
$$

where $\Delta(\mathfrak{g}(r))$ is the set of roots $\alpha$ so that $\mathfrak{g}_{\alpha} \subset \mathfrak{g}(r)$, and where $X_{\gamma_{j}}^{*}$ are the elements in $\mathfrak{g}(-r)$ dual to $X_{\gamma_{j}}$ with respect to the Killing form; namely, $X_{\gamma_{j}}^{*}\left(X_{\gamma_{t}}\right):=$ $\kappa\left(X_{\gamma_{j}}^{*}, X_{\gamma_{t}}\right)=\delta_{\gamma_{j}, \gamma_{t}}$ with $\delta_{\gamma_{j}, \gamma_{t}}$ the Kronecker delta.

We shall check in Lemma 3.3 that these maps are indeed $L$-equivariant. Observe that, by the standard argument, the element $\omega$ is independent of a choice of a basis for $\mathfrak{g}(r)$ and the dual basis for $\mathfrak{g}(-r)$.

Lemma 3.2. Let $\mathfrak{g}=\bigoplus_{j=-r}^{r} \mathfrak{g}(j)$ be a graded complex simple Lie algebra with $\mathfrak{g}(1) \neq 0$ and $G$ be a complex analytic group with Lie algebra $\mathfrak{g}$. If $L$ is the analytic subgroup of $G$ with Lie algebra $\mathfrak{g}(0)$ and $\omega$ is as in Definition 3.1 , then, for all $l \in L$,

$$
(\operatorname{Ad}(l) \otimes \operatorname{Ad}(l)) \omega=\omega
$$

Proof. If $g \in L$, then $\left\{\operatorname{Ad}(l) X_{\gamma_{j}} \mid \gamma_{j} \in \Delta(\mathfrak{g}(r))\right\}$ forms a basis for $\mathfrak{g}(r)$. It also holds that $\left\{\operatorname{Ad}(l) X_{\gamma_{j}}^{*} \mid \gamma_{j} \in \Delta(\mathfrak{g}(r))\right\}$ is the dual basis for $\mathfrak{g}(-r)$ with respect to the Killing form. Now the assertion follows from the property that $\omega$ is independent of a choice of a basis for $\mathfrak{g}(r)$ and the dual basis for $\mathfrak{g}(-r)$.

Lemma 3.3. Let $\mathfrak{g}=\bigoplus_{j=-r}^{r} \mathfrak{g}(j), G$, and $L$ be as in Lemma 3.2. For all $l \in L$, $X \in \mathfrak{g}(1)$, and for $0 \leq k \leq 2 r$, we have

$$
\tau_{k}(\operatorname{Ad}(l) X)=(\operatorname{Ad}(l) \otimes \operatorname{Ad}(l)) \tau_{k}(X)
$$


Proof. For $l \in L$, we have

$$
\begin{aligned}
\tau_{k}(\operatorname{Ad}(l) X) & =\frac{1}{k !} \sum_{\gamma_{j} \in \Delta(\mathfrak{z}(\mathfrak{n}))} \operatorname{ad}(\operatorname{Ad}(l)(X))^{k}\left(X_{\gamma_{j}}^{*}\right) \otimes X_{\gamma_{j}} \\
& =\frac{1}{k !} \sum_{\gamma_{j} \in \Delta(\mathfrak{z}(\mathfrak{n}))} \operatorname{Ad}(l)\left(\operatorname{ad}(X)^{k}\left(\operatorname{Ad}\left(l^{-1}\right) X_{\gamma_{j}}^{*}\right)\right) \otimes X_{\gamma_{j}} \\
& =(\operatorname{Ad}(l) \otimes \operatorname{Ad}(l))\left(\frac{1}{k !} \sum_{\gamma_{j} \in \Delta(\mathfrak{z}(\mathfrak{n}))} \operatorname{ad}(X)^{k}\left(\operatorname{Ad}\left(l^{-1}\right) X_{\gamma_{j}}^{*}\right) \otimes \operatorname{Ad}\left(l^{-1}\right)\left(X_{\gamma_{j}}\right)\right) \\
& =(\operatorname{Ad}(l) \otimes \operatorname{Ad}(l))\left(\frac{1}{k !}\left(\operatorname{ad}(X)^{k} \otimes \operatorname{Id}\right) \omega\right) \\
& =(\operatorname{Ad}(l) \otimes \operatorname{Ad}(l)) \tau_{k}(X) .
\end{aligned}
$$

Note that Lemma 3.2 is applied from line four to line five.

Now we are going to build the systems of differential operators in $\mathbb{D}\left(\mathcal{L}_{s}\right)^{\overline{\mathfrak{n}}}$ that we will study. Observe that, as $\tau_{k}: \mathfrak{g}(1) \rightarrow \mathfrak{g}(-r+k) \otimes \mathfrak{g}(r)=: W$ are $L$-equivariant polynomial maps of degree $k$, the maps $\tau_{k}$ can be thought of as elements in $\left(\mathcal{P}^{k}(\mathfrak{g}(1)) \otimes W\right)^{L}$, where $\mathcal{P}^{k}(\mathfrak{g}(1))$ denotes the space of homogeneous polynomials on $\mathfrak{g}(1)$ of degree $k$. Then the isomorphism $\left(\mathcal{P}^{k}(\mathfrak{g}(1)) \otimes W\right)^{L} \cong \operatorname{Hom}_{L}\left(W^{*}, \mathcal{P}^{k}(\mathfrak{g}(1))\right)$ yields the $L$-intertwining operators $\tilde{\tau}_{k}$ that are given by

$$
\tilde{\tau}_{k}\left(Y^{*}\right)(X)=Y^{*}\left(\tau_{k}(X)\right)
$$

where $W^{*}$ is the dual module of $W$ with respect to the Killing form. For each $Y^{*} \in$ $W^{*}$, we have $\tilde{\tau}_{k}\left(Y^{*}\right) \in \mathcal{P}^{k}(\mathfrak{g}(1)) \cong \operatorname{Sym}^{k}(\mathfrak{g}(-1))$. We define differential operators in $\mathbb{D}\left(\mathcal{L}_{s}\right)^{\overline{\mathfrak{n}}}$ from $\tilde{\tau}_{k}\left(Y^{*}\right)$. This is done as follows. Let $\sigma: \operatorname{Sym}^{k}(\mathfrak{g}(-1)) \rightarrow \mathcal{U}(\overline{\mathfrak{n}})$ be the symmetrization operator. Identify $\mathcal{U}(\overline{\mathfrak{n}})$ with $\mathbb{D}\left(\mathcal{L}_{s}\right)^{\overline{\mathfrak{n}}}$ by making $\overline{\mathfrak{n}}$ act on $C^{\infty}\left(\bar{N}_{0}, \mathbb{C}_{\chi^{s}}\right)$ via right differentiation $R$. Then we have a composition of linear maps

$$
W^{*} \stackrel{\tilde{\tau}_{k}}{\rightarrow} \mathcal{P}^{k}(\mathfrak{g}(1)) \cong \operatorname{Sym}^{k}(\mathfrak{g}(-1)) \stackrel{\sigma}{\rightarrow} \mathcal{U}(\overline{\mathfrak{n}}) \stackrel{R}{\rightarrow} \mathbb{D}\left(\mathcal{L}_{s}\right)^{\overline{\mathfrak{n}}}
$$

For $Y^{*} \in W^{*}$, we define a differential operator $\Omega_{k}\left(Y^{*}\right) \in \mathbb{D}\left(\mathcal{L}_{s}\right)^{\overline{\mathfrak{n}}}$ by

$$
\Omega_{k}\left(Y^{*}\right)=R \circ \sigma \circ \tilde{\tau}_{k}\left(Y^{*}\right) .
$$

As we will work with irreducible systems we need to be a little more careful with our construction; in particular, we need to take an irreducible constituent of $\mathfrak{g}(-r+k)^{*} \otimes \mathfrak{g}(r)^{*}$. Let $\mathfrak{g}(-r+k) \otimes \mathfrak{g}(r)=V_{1} \oplus \cdots \oplus V_{m}$ be the irreducible decomposition of $\mathfrak{g}(-r+k) \otimes \mathfrak{g}(r)$ as an $L$-module, and let $\mathfrak{g}(-r+k)^{*} \otimes \mathfrak{g}(r)^{*}=$ $V_{1}^{*} \oplus \cdots \oplus V_{m}^{*}$ be the corresponding irreducible decomposition of $\mathfrak{g}(-r+k)^{*} \otimes \mathfrak{g}(r)^{*}$, where $\mathfrak{g}(j)^{*}$ are the dual spaces of $\mathfrak{g}(j)$ with respect to the Killing form. For each irreducible constituent $V_{j}^{*}$ of $\mathfrak{g}(-r+k)^{*} \otimes \mathfrak{g}(r)^{*}$, there exists an $L$-intertwining operator $\left.\tilde{\tau}_{k}\right|_{V_{j}^{*}} \in \operatorname{Hom}_{L}\left(V_{j}^{*}, \mathcal{P}^{k}(\mathfrak{g}(1))\right)$ given as in (3.4). Then we define a linear operator $\left.\Omega_{k}\right|_{V_{j}^{*}}: V_{j}^{*} \rightarrow \mathbb{D}\left(\mathcal{L}_{s}\right)^{\overline{\mathfrak{n}}}$ by

$$
\left.\Omega_{k}\right|_{V_{j}^{*}}=\left.R \circ \sigma \circ \tilde{\tau}_{k}\right|_{V_{j}^{*}}
$$

Since, for $Y^{*} \in V_{j}^{*}$, we have $\left.\Omega_{k}\right|_{V_{j}^{*}}\left(Y^{*}\right)=\Omega_{k}\left(Y^{*}\right)$ as a differential operator, we simply write $\Omega_{k}\left(Y^{*}\right)$ for the differential operator arising from $Y^{*} \in V_{j}^{*}$. 
Definition 3.5. If $V^{*}$ is an irreducible constituent of $\mathfrak{g}(-r+k)^{*} \otimes \mathfrak{g}(r)^{*}$ so that $\left.\tilde{\tau}_{k}\right|_{V^{*}} \not \equiv 0$, then a list of differential operators $D_{1}, \ldots, D_{n} \in \mathbb{D}\left(\mathcal{L}_{s}\right)^{\overline{\mathfrak{n}}}$ is called the $\left.\Omega_{k}\right|_{V^{*}}$ system if it is equivalent (see Definition 2.4) to a list of differential operators

$$
\Omega_{k}\left(Y_{1}^{*}\right), \ldots, \Omega_{k}\left(Y_{n}^{*}\right),
$$

where $\left\{Y_{1}^{*}, \ldots, Y_{n}^{*}\right\}$ is a basis for $V^{*}$.

We also simply refer each $\left.\Omega_{k}\right|_{W^{*}}$ system to an $\Omega_{k}$ system. We want to remark that the construction of the $\Omega_{k}$ systems might require additional modification to secure the conformal invariance. See Section 6 in [1] and Section 3 in 21] for the modification for the $\Omega_{3}$ systems of the parabolic subalgebra of Heisenberg type.

It is important to notice that it is not necessary for the $\Omega_{k}$ systems to be conformally invariant; their conformal invariance strongly depends on the complex parameter $s$ for the line bundle $\mathcal{L}_{s}$. So, we give the following definition.

Definition 3.7. Let $V^{*}$ be an irreducible constituent of $\mathfrak{g}(-r+k)^{*} \otimes \mathfrak{g}(r)^{*}$. Then we say that the $\left.\Omega_{k}\right|_{V^{*}}$ system has special value $s_{0}$ if the system is conformally invariant on the line bundle $\mathcal{L}_{s_{0}}$.

Note that, as the opposite parabolic $\bar{Q}_{0}=L_{0} \bar{N}_{0}$ is chosen in [1], our special values $s_{0}$ are of the form $s_{0}=-s_{0}^{\prime}$, where $s_{0}^{\prime}$ are the special values shown in [1].

Observe that the linear operator $\left.\Omega_{k}\right|_{V^{*}}: V^{*} \rightarrow \mathbb{D}\left(\mathcal{L}_{s}\right)^{\overline{\mathfrak{n}}}$ is an $L_{0}$-intertwining operator with respect to the action given in (2.11); in particular, the $\left.\Omega_{k}\right|_{V^{*}}$ system is $L_{0}$-stable (see Definition 2.12). Indeed, one can check that we have $l$. $R(u)=R(\operatorname{Ad}(l) u)$ for $l \in L_{0}$ and $u \in \mathcal{U}(\overline{\mathfrak{n}})$. This action stabilizes the subspace $\mathbb{D}\left(\mathcal{L}_{s}\right)^{\overline{\mathfrak{n}}}$. With the adjoint action of $L_{0}$ on $\mathcal{U}(\overline{\mathfrak{n}})$, the linear isomorphism $\mathcal{U}(\overline{\mathfrak{n}}) \stackrel{R}{\rightarrow} \mathbb{D}\left(\mathcal{L}_{s}\right)^{\overline{\mathfrak{n}}}$ is then $L_{0}$-equivariant. It is clear that each map in $V^{*} \stackrel{\left.\tilde{\tau}_{k}\right|_{\zeta^{*}}}{\longrightarrow}$ $\mathcal{P}^{k}(\mathfrak{g}(1)) \cong \operatorname{Sym}^{k}(\mathfrak{g}(-1)) \stackrel{\sigma}{\hookrightarrow} \mathcal{U}(\overline{\mathfrak{n}})$ is $L_{0}$-equivariant with respect to the natural actions of $L_{0}$ on each space, which are induced by the adjoint action of $L_{0}$ on $\mathfrak{g}$. Therefore, with the $L_{0}$-action (2.11), the operator $\left.\Omega_{k}\right|_{V^{*}}: V^{*} \rightarrow \mathbb{D}\left(\mathcal{L}_{s}\right)^{\overline{\mathfrak{n}}}$ is an $L_{0}$-intertwining operator. Now we summarize some properties of the $\left.\Omega_{k}\right|_{V^{*}}$ system.

Remark 3.8. It follows from the definition and observation above that the $\left.\Omega_{k}\right|_{V^{*}}$ system satisfies the following properties:

(1) The $\left.\Omega_{k}\right|_{V^{*}}$ system satisfies condition (S1) of Definition 2.3

(2) When the $\left.\Omega_{k}\right|_{V^{*}}$ system is conformally invariant, then it is an irreducible, straight, and $L_{0}$-stable system. By Proposition 2.14 it is also a homogeneous system.

3.2. Computations involving the $\Omega_{k}$ systems. We are going to show two technical lemmas that will be used in Section 7 . For $D \in \mathbb{D}\left(\mathcal{L}_{s}\right)$, we denote by $D_{\bar{n}}$ the linear functional $f \mapsto(D \bullet f)(\bar{n})$ for $f \in C^{\infty}\left(\bar{N}_{0}, \mathbb{C}_{\chi^{s}}\right)$. A simple observation shows that $\left(D_{1} D_{2}\right)_{\bar{n}}=\left(D_{1}\right)_{\bar{n}} D_{2}$ for $D_{1}, D_{2} \in \mathbb{D}\left(\mathcal{L}_{s}\right)$; in particular, if $\left(D_{1}\right)_{\bar{n}}=0$, then $\left[D_{1}, D_{2}\right]_{\bar{n}}=-\left(D_{2}\right)_{\bar{n}} D_{1}$.

Lemma 3.9. Suppose that $V^{*}$ is an irreducible constituent of $\mathfrak{g}(-r+k)^{*} \otimes \mathfrak{g}(r)^{*}$. Let $X_{1}, X_{2} \in \mathfrak{g}$ and $Y_{1}^{*}, \ldots, Y_{n}^{*} \in V^{*}$. If $\pi_{s}\left(X_{1}\right)_{e}=0$ and if we have $\left[\pi_{s}\left(X_{i}\right), \Omega_{k}\left(Y_{t}^{*}\right)\right]_{e}$ $\in \operatorname{span}_{\mathbb{C}}\left\{\Omega_{k}\left(Y_{j}^{*}\right)_{e} \mid j=1, \ldots, n\right\}$ for $i=1,2$, then

$$
\left[\pi_{s}\left(X_{1}\right),\left[\pi_{s}\left(X_{2}\right), \Omega_{k}\left(Y_{t}^{*}\right)\right]\right]_{e} \in \operatorname{span}_{\mathbb{C}}\left\{\Omega_{k}\left(Y_{1}^{*}\right)_{e}, \ldots, \Omega_{k}\left(Y_{n}^{*}\right)_{e}\right\} .
$$


Proof. Observe that $\left[\pi_{s}\left(X_{1}\right),\left[\pi_{s}\left(X_{2}\right), \Omega_{k}\left(Y_{t}^{*}\right)\right]\right]$ is

$$
\pi_{s}\left(X_{1}\right)\left[\pi_{s}\left(X_{2}\right), \Omega_{k}\left(Y_{t}^{*}\right)\right]-\left[\pi_{s}\left(X_{2}\right), \Omega_{k}\left(Y_{t}^{*}\right)\right] \pi_{s}\left(X_{1}\right) .
$$

Since, by assumption, we have $\pi_{s}\left(X_{1}\right)_{e}=0$, the first term is zero at $e$. By assumption, the bracket $\left[\pi_{s}\left(X_{2}\right), \Omega_{k}\left(Y_{t}^{*}\right)\right]_{e}$ is a linear combination of $\Omega_{k}\left(Y_{1}^{*}\right)_{e}, \ldots, \Omega_{k}\left(Y_{n}^{*}\right)_{e}$ over $\mathbb{C}$. So it may be written as $\left[\pi_{s}\left(X_{2}\right), \Omega_{k}\left(Y_{t}^{*}\right)\right]_{e}=\sum_{j=1}^{n} a_{j t} \Omega_{k}\left(Y_{j}^{*}\right)_{e}$ with $a_{j t} \in \mathbb{C}$. Then, the second term in (3.11) evaluates to $-\sum_{j=1}^{n} a_{j t} \Omega_{k}\left(Y_{j}^{*}\right)_{e} \pi_{s}\left(X_{1}\right)$ at the identity $e$. Since $\left(\pi_{s}\left(X_{1}\right) \Omega_{k}\left(Y_{j}^{*}\right)\right)_{e}=\pi_{s}\left(X_{1}\right)_{e} \Omega_{k}\left(Y_{j}^{*}\right)=0$, we obtain

$$
\begin{aligned}
{\left[\pi_{s}\left(X_{1}\right),\left[\pi_{s}\left(X_{2}\right), \Omega_{k}\left(Y_{t}^{*}\right)\right]\right]_{e} } & =-\sum_{j=1}^{n} a_{j t} \Omega_{k}\left(Y_{j}^{*}\right) \pi_{e}\left(X_{1}\right) \\
& =-\sum_{j=1}^{n} a_{j t}\left[\pi_{s}\left(X_{1}\right), \Omega_{k}\left(Y_{j}^{*}\right)\right]_{e} .
\end{aligned}
$$

Now the proposed result follows from the assumption that $\left[\pi_{s}\left(X_{1}\right), \Omega_{k}\left(Y_{t}^{*}\right)\right]_{e}$ is a linear combination of $\Omega_{k}\left(Y_{j}^{*}\right)_{e}$ over $\mathbb{C}$.

If $\Delta^{+}(\mathfrak{l})$ is the set of positive roots in $\mathfrak{l}$, then we set

$$
\mathfrak{u}_{\mathfrak{l}}=\bigoplus_{\Delta^{+}(\mathfrak{l})} \mathfrak{g}_{\alpha} \text { and } \overline{\mathfrak{u}}_{\mathfrak{l}}=\bigoplus_{\Delta^{+}(\mathfrak{l})} \mathfrak{g}_{-\alpha} .
$$

Lemma 3.12. Suppose that $\mathfrak{g}(1)$ is irreducible and that $V^{*}$ is an irreducible constituent of $\mathfrak{g}(-r+k)^{*} \otimes \mathfrak{g}(r)^{*}$. Let $X_{h}$ be a highest weight vector for $\mathfrak{g}(1)$ and $Y_{l}^{*}$ be a lowest weight vector for $V^{*}$. If

$$
\left[\pi_{s}\left(X_{h}\right), \Omega_{k}\left(Y_{l}^{*}\right)\right]_{e} \in \operatorname{span}_{\mathbb{C}}\left\{\Omega_{k}\left(Y_{1}^{*}\right)_{e}, \ldots, \Omega_{k}\left(Y_{n}^{*}\right)_{e}\right\}
$$

with $\left\{Y_{1}^{*}, \ldots, Y_{n}^{*}\right\}$ a basis for $V^{*}$, then, for any $X \in \mathfrak{g}(1)$ and $Y^{*} \in V^{*}$,

$$
\left[\pi_{s}(X), \Omega_{k}\left(Y^{*}\right)\right]_{e} \in \operatorname{span}_{\mathbb{C}}\left\{\Omega_{k}\left(Y_{1}^{*}\right)_{e}, \ldots, \Omega_{k}\left(Y_{n}^{*}\right)_{e}\right\} .
$$

Proof. Set $E=\operatorname{span}_{\mathbb{C}}\left\{\Omega_{k}\left(Y_{1}^{*}\right)_{e}, \ldots, \Omega_{k}\left(Y_{n}^{*}\right)_{e}\right\}$. We first show that for each $X \in$ $\mathfrak{g}(1)$,

$$
\left[\pi_{s}(X), \Omega_{k}\left(Y_{l}^{*}\right)\right]_{e} \in E .
$$

Observe that since $(L, \mathfrak{g}(1))$ is assumed to be irreducible, the $L$-module $\mathfrak{g}(1)$ is given by $\mathfrak{g}(1)=\mathcal{U}\left(\overline{\mathfrak{u}}_{\mathfrak{l}}\right) X_{h}$. Then, as $\pi_{s}$ is linear on $\mathfrak{g}(1)$, it suffices to show that (3.13) holds when $X=\bar{u}_{k} \cdot X_{h}$ with $\bar{u}_{k}$ a monomial in $\mathcal{U}\left(\overline{\mathfrak{u}}_{\mathfrak{l}}\right)$. This is done by induction on the order of $\bar{u}_{k}$. Indeed, the proof is clear once we show that (3.13) holds for $X=\bar{Z} \cdot X_{h}=\left[\bar{Z}, X_{h}\right]$ with $\bar{Z} \in \overline{\mathfrak{u}}_{\mathfrak{l}}$.

By the Jocobi identity, the commutator $\left[\pi_{s}\left(\left[\bar{Z}, X_{h}\right]\right), \Omega_{k}\left(Y_{l}^{*}\right)\right]$ is

$$
\begin{aligned}
& {\left[\pi_{s}\left(\left[\bar{Z}, X_{h}\right]\right), \Omega_{k}\left(Y_{l}^{*}\right)\right]} \\
& =\left[\pi_{s}(\bar{Z}),\left[\pi_{s}\left(X_{h}\right), \Omega_{k}\left(Y_{l}^{*}\right)\right]\right]-\left[\pi_{s}\left(X_{h}\right),\left[\pi_{s}(\bar{Z}), \Omega_{k}\left(Y_{l}^{*}\right)\right]\right] .
\end{aligned}
$$

By the l-equivariance of the operator $\Omega_{k}: V^{*} \rightarrow \mathbb{D}\left(\mathcal{L}_{s}\right)^{\overline{\mathfrak{n}}}$, it follows that

$$
\left[\pi_{s}(\bar{Z}), \Omega_{k}\left(Y_{l}^{*}\right)\right]=\Omega_{k}\left(\left[\bar{Z}, Y_{l}^{*}\right]\right) .
$$

Since $\bar{Z} \in \overline{\mathfrak{u}}_{\mathfrak{l}}$ and $Y_{l}^{*}$ is a lowest weight vector, we have $\Omega_{k}\left(\left[\bar{Z}, Y_{l}^{*}\right]\right)=0$, and so is the second term of the right hand side of (3.14). Thus we have

$$
\left[\pi_{s}\left(\left[\bar{Z}, X_{h}\right]\right), \Omega_{k}\left(Y_{l}^{*}\right)\right]_{e}=\left[\pi_{s}(\bar{Z}),\left[\pi_{s}\left(X_{h}\right), \Omega_{k}\left(Y_{l}^{*}\right)\right]\right]_{e} .
$$


Now, by hypotheses and the l-equivariance of $\Omega_{k}$, it follows that

$$
\left[\pi_{s}\left(X_{h}\right), \Omega_{k}\left(Y_{l}^{*}\right)\right]_{e},\left[\pi_{s}(\bar{Z}), \Omega_{k}\left(Y_{l}^{*}\right)\right]_{e} \in E .
$$

As $\bar{Z} \in \overline{\mathfrak{u}}_{\mathfrak{l}}$, by (2.9), we have $\pi_{s}(\bar{Z})_{e}=0$. Thus it follows from Lemma 3.9 that we have $\left[\pi_{s}(\bar{Z}),\left[\pi_{s}\left(X_{h}\right), \Omega_{k}\left(Y_{l}^{*}\right)\right]\right]_{e} \in E$. Therefore, $\left[\pi_{s}\left(\left[\bar{Z}, X_{h}\right]\right), \Omega_{k}\left(Y_{l}^{*}\right)\right]_{e} \in E$ by (3.15).

Next we show that for any $X \in \mathfrak{g}(1)$ and $Y^{*} \in V^{*}$,

$$
\left[\pi_{s}(X), \Omega_{k}\left(Y^{*}\right)\right]_{e} \in E .
$$

Once again since $V^{*}$ is irreducible, it is given by $V^{*}=\mathcal{U}\left(\mathfrak{u}_{\mathfrak{l}}\right) Y_{l}^{*}$. As before, it is enough to show that (3.16) holds for $Y^{*}=Z \cdot Y_{l}^{*}$ with $Z \in \mathfrak{u}_{\text {. }}$. Since $\Omega_{k}\left(Z \cdot Y_{l}^{*}\right)=$ $\left[\pi_{s}(Z), \Omega_{k}\left(Y_{l}^{*}\right)\right]$, by the Jacobi identity, the commutator $\left[\pi_{s}(X), \Omega_{k}\left(Z \cdot Y_{l}^{*}\right)\right]$ is

$$
\begin{aligned}
& {\left[\pi_{s}(X), \Omega_{k}\left(Z \cdot Y_{l}^{*}\right)\right]} \\
& =\left[\pi_{s}(Z),\left[\pi_{s}(X), \Omega_{k}\left(Y_{l}^{*}\right)\right]\right]-\left[\left[\pi_{s}(Z), \pi_{s}(X)\right], \Omega_{k}\left(Y_{l}^{*}\right)\right] .
\end{aligned}
$$

We showed above that $\left[\pi_{s}(X), \Omega_{k}\left(Y_{l}^{*}\right)\right]_{e} \in E$. Since we have $\pi_{s}(Z)_{e}=0$ and $\left[\pi_{s}(Z), \Omega_{k}\left(Y_{l}^{*}\right)\right]_{e} \in E$, by Lemma 3.9] the first term of the right hand side of (3.17) satisfies

$$
\left[\pi_{s}(Z),\left[\pi_{s}(X), \Omega_{k}\left(Y_{l}^{*}\right)\right]\right]_{e} \in E .
$$

Moreover, as $\left[\pi_{s}(Z), \pi_{s}(X)\right]=\pi_{s}([Z, X])$ with $[Z, X] \in \mathfrak{g}(1)$, by what we have shown above, the second term satisfies

$$
\left[\left[\pi_{s}(Z), \pi_{s}(X)\right], \Omega_{k}\left(Y_{l}^{*}\right)\right]_{e} \in E .
$$

Hence, $\left[\pi_{s}(X), \Omega_{k}\left(Z \cdot Y_{l}^{*}\right)\right]_{e} \in E$.

\section{Parabolic SUbalgebras AND $\mathbb{Z}$-GRADingS}

It has been observed in Subsection 3.1 that the $\mathbb{Z}$-grading $\mathfrak{g}=\bigoplus_{j=-r}^{r} \mathfrak{g}(j)$ on $\mathfrak{g}$ and parabolic subalgebra $\mathfrak{q}$ play a role in constructing the $\Omega_{k}$ systems. In this section we observe these in detail for $\mathfrak{q}$ a maximal parabolic subalgebra of quasiHeisenberg type. The $\Omega_{1}$ system and $\Omega_{2}$ systems of those parabolic subalgebras will be constructed in Section 5 and Section 7 , respectively.

4.1. Parabolic subalgebras of $k$-step nilpotent type. Let $\mathfrak{r}$ be any non-zero Lie algebra. Put $\mathfrak{r}_{0}=\mathfrak{r}, \mathfrak{r}_{1}=[\mathfrak{r}, \mathfrak{r}]$, and $\mathfrak{r}_{k}=\left[\mathfrak{r}, \mathfrak{r}_{k-1}\right]$ for $k \in \mathbb{Z}_{>0}$. We call $\mathfrak{r}_{k}$ the $k$ th step of $\mathfrak{r}$ for $k \in \mathbb{Z}_{\geq 0}$. The Lie algebra $\mathfrak{r}$ is called nilpotent if $\mathfrak{r}_{k}=0$ for some $k$, and it is called $k$-step nilpotent if $\mathfrak{r}_{k-1} \neq 0$ and $\mathfrak{r}_{k}=0$. In particular, if $[\mathfrak{r}, \mathfrak{r}]=0$, then $\mathfrak{r}$ is called abelian, and if $\operatorname{dim}([\mathfrak{r}, \mathfrak{r}])=1$, then $\mathfrak{r}$ is called Heisenberg. If $[\mathfrak{r},[\mathfrak{r}, \mathfrak{r}]]=0$ and $\operatorname{dim}([\mathfrak{r}, \mathfrak{r}])>1$, then we call $\mathfrak{r}$ quasi-Heisenberg. Note that $\mathfrak{r}$ is Heisenberg if and only if its center $\mathfrak{z}(\mathfrak{r})$ is one-dimensional. If the nilpotent radical $\mathfrak{n}$ of a parabolic subalgebra $\mathfrak{q}=\mathfrak{l} \oplus \mathfrak{n}$ is $k$-step nilpotent (resp. abelian, Heisenberg, or quasi-Heisenberg), then we say that $\mathfrak{q}$ is of $k$-step nilpotent (resp. abelian, Heisenberg, or quasi-Heisenberg) type.

To build the $\Omega_{1}$ system and $\Omega_{2}$ systems of a maximal parabolic subalgebra $\mathfrak{q}$ of quasi-Heisenberg type, it is convenient to classify the parabolic subalgebras $\mathfrak{q}$ of $k$-step nilpotent type by the subsets of simple roots. If $\beta=\sum_{\alpha \in \Pi} m_{\alpha} \alpha \in \sum_{\alpha \in \Pi} \mathbb{Z} \alpha$, then we say that $\left|m_{\alpha}\right|$ are the multiplicities of $\alpha$ in $\beta$. 
Proposition 4.1. Let $\mathfrak{g}$ be a complex simple Lie algebra with highest root $\gamma$, and $\mathfrak{q}_{S}=\mathfrak{l} \oplus \mathfrak{n}$ be the parabolic subalgebra of $\mathfrak{g}$ that is parametrized by $S$ with $S=$ $\left\{\alpha_{i_{1}}, \ldots, \alpha_{i_{r}}\right\} \subset \Pi$. Then $\mathfrak{n}$ is $k$-step nilpotent if and only if $k=m_{i_{1}}+m_{i_{2}}+\cdots+$ $m_{i_{r}}$, where $m_{i_{j}}$ are the multiplicities of $\alpha_{i_{j}}$ in $\gamma$.

Proof. As this is a well-known fact, we omit a proof. (For a proof, see for instance Section 3.1 of [20].)

The following observation would be useful when we consider parabolic subalgebras of $k$-step nilpotent type. First, observe that, by the one-to-one correspondence between the standard parabolic subalgebras $\mathfrak{q}_{S}$ and the subsets $S \subset \Pi$, we can associate subdiagrams of Dynkin diagrams to parabolic subalgebras $\mathfrak{q}_{S}$. The subdiagrams that associate to $\mathfrak{q}_{S}$ are obtained by deleting the nodes of the Dynkin diagram of $\mathfrak{g}$ that correspond to the simple roots in $S$, and the edges incident on them. We call such subdiagrams deleted Dynkin diagrams. With the multiplicities of simple roots in the highest root of $\mathfrak{g}$ in hand, by Proposition 4.1, we then see the number of steps of nilradical $\mathfrak{n}$ of $\mathfrak{q}_{S}$ from the deleted Dynkin diagram. Table 1 shows the multiplicities of the simple roots in the highest root $\gamma$. We use the Bourbaki conventions [3] for the labels of the simple roots.

TABLE 1. Highest roots

\begin{tabular}{cc}
\hline Type & Highest root \\
\hline$A_{n}$ & $\alpha_{1}+\alpha_{2}+\cdots+\alpha_{n}$ \\
$B_{n}$ & $\alpha_{1}+2 \alpha_{2}+2 \alpha_{3}+\cdots+2 \alpha_{n}$ \\
$C_{n}$ & $2 \alpha_{1}+2 \alpha_{2}+\cdots+2 \alpha_{n-1}+1 \alpha_{n}$ \\
$D_{n}$ & $\alpha_{1}+2 \alpha_{2}+2 \alpha_{3}+\cdots+2 \alpha_{n-2}+\alpha_{n-1}+\alpha_{n}$ \\
$E_{6}$ & $\alpha_{1}+2 \alpha_{2}+2 \alpha_{3}+3 \alpha_{4}+2 \alpha_{5}+\alpha_{6}$ \\
$E_{7}$ & $2 \alpha_{1}+2 \alpha_{2}+3 \alpha_{3}+4 \alpha_{4}+3 \alpha_{5}+2 \alpha_{6}+\alpha_{7}$ \\
$E_{8}$ & $2 \alpha_{1}+3 \alpha_{2}+4 \alpha_{3}+6 \alpha_{4}+5 \alpha_{5}+4 \alpha_{6}+3 \alpha_{7}+2 \alpha_{8}$ \\
$F_{4}$ & $2 \alpha_{1}+3 \alpha_{2}+4 \alpha_{3}+2 \alpha_{4}$ \\
$G_{2}$ & $3 \alpha_{1}+2 \alpha_{2}$ \\
\hline
\end{tabular}

Example 4.2 below describes the deleted Dynkin diagram of a given parabolic $\mathfrak{q}_{S}$ and how we read the diagram. For simplicity, we depict deleted Dynkin diagrams by crossing out the deleted nodes.

Example 4.2. Let $\mathfrak{g}=\mathfrak{s l}(6, \mathbb{C})$. The Dynkin diagram is

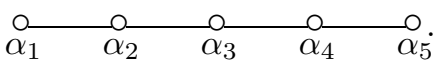

Choose $S=\left\{\alpha_{2}, \alpha_{4}\right\} \subset \Pi$. Then the deleted Dynkin diagram of parabolic subalgebra $\mathfrak{q}_{S}$ corresponding to the subset $S$ is

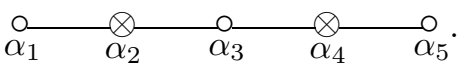

Moreover, by Table 1 the multiplicity of each simple root in the highest root of $\mathfrak{g}$ is 1 . Thus, $\mathfrak{q}_{S}$ is a parabolic subalgebra of two-step nilpotent type.

By the above observation we often refer to parabolic subalgebras $\mathfrak{q}_{S}$ by their corresponding subset $S$ of simple roots. To this end, we are going to define classification types of parabolics $\mathfrak{q}_{S}$. In Definition 4.3 below, we mean by classification type $\mathcal{T}$ of $\mathfrak{g}$ type $A_{n}, B_{n}, C_{n}, D_{n}, E_{6}, E_{7}, E_{8}, F_{4}$, or $G_{2}$. 
Definition 4.3. If $\mathfrak{g}$ is a complex simple Lie algebra of classification type $\mathcal{T}$ and if $S$ is a subset of $\Pi$ of simple roots, then we say that a parabolic subalgebra $\mathfrak{q}_{S}$ of $\mathfrak{g}$ is of type $\mathcal{T}(S)$, or type $\mathcal{T}\left(i_{1}, \ldots, i_{k}\right)$ if $S=\left\{\alpha_{i_{1}}, \ldots, \alpha_{i_{k}}\right\}$.

For example, the parabolic subalgebra $\mathfrak{q}_{S}$ in Example 4.2 is of type $A_{5}(2,4)$. Any maximal parabolic subalgebra is of type $\mathcal{T}(i)$ for some $\alpha_{i} \in \Pi$.

4.2. Maximal parabolic subalgebra $\mathfrak{q}$ of quasi-Heisenberg type. Now we observe the 2-grading $\mathfrak{g}=\bigoplus_{j=-2}^{2} \mathfrak{g}(j)$ on $\mathfrak{g}$ that is induced from a maximal parabolic subalgebra $\mathfrak{q}$ of quasi-Heisenberg type.

Assume that $\mathfrak{g}$ has rank greater than one and that $\alpha_{\mathfrak{q}}$ is a simple root, so that the parabolic subalgebra $\mathfrak{q}=\mathfrak{q}_{\left\{\alpha_{\mathfrak{q}}\right\}}=\mathfrak{l} \oplus \mathfrak{n}$ parameterized by $\alpha_{\mathfrak{q}}$ is a maximal parabolic subalgebra of quasi-Heisenberg type, namely, $[\mathfrak{n},[\mathfrak{n}, \mathfrak{n}]]=0$ and $\operatorname{dim}([\mathfrak{n}, \mathfrak{n}])>1$. Let $\langle\cdot, \cdot\rangle$ be the inner product induced on $\mathfrak{h}^{*}$ corresponding to the Killing form $\kappa$. Write $\|\alpha\|^{2}=\langle\alpha, \alpha\rangle$ for $\alpha \in \Delta$. The coroot of $\alpha$ is $\alpha^{\vee}=2 \alpha /\langle\alpha, \alpha\rangle$.

Recall from Section 2 that $\lambda_{\mathfrak{q}}$ denotes the fundamental weight for $\alpha_{\mathfrak{q}}$. As $\Delta(\mathfrak{l})=$ $\left\{\alpha \in \Delta \mid \alpha \in \operatorname{span}\left(\Pi \backslash\left\{\alpha_{\mathfrak{q}}\right\}\right)\right\}$ and $\Delta(\mathfrak{n})=\Delta^{+} \backslash \Delta(\mathfrak{l})$, we have

$$
\left\langle\lambda_{\mathfrak{q}}, \beta\right\rangle \begin{cases}=0 & \text { if } \beta \in \Delta(\mathfrak{l}), \\ >0 & \text { if } \beta \in \Delta(\mathfrak{n}) .\end{cases}
$$

Observe that if $H_{\lambda_{\mathfrak{q}}} \in \mathfrak{h}$ is defined by $\kappa\left(H, H_{\lambda_{\mathfrak{q}}}\right)=\lambda_{\mathfrak{q}}(H)$ for all $H \in \mathfrak{h}$ and if

$$
H_{\mathfrak{q}}=\frac{2}{\left\|\alpha_{\mathfrak{q}}\right\|^{2}} H_{\lambda_{\mathfrak{q}}}
$$

then $\beta\left(H_{\mathfrak{q}}\right)$ is the multiplicity of $\alpha_{\mathfrak{q}}$ in $\beta$. In particular, it follows from Proposition 4.1 that for $\beta \in \Delta^{+}, \beta\left(H_{\mathfrak{q}}\right)$ can only take the values of 0,1 , or 2 . Therefore, if $\mathfrak{g}(j)$ denotes the $j$-eigenspace of $\operatorname{ad}\left(H_{\mathfrak{q}}\right)$, then the action of $\operatorname{ad}\left(H_{\mathfrak{q}}\right)$ on $\mathfrak{g}$ induces a 2-grading

$$
\mathfrak{g}=\mathfrak{g}(-2) \oplus \mathfrak{g}(-1) \oplus \mathfrak{g}(0) \oplus \mathfrak{g}(1) \oplus \mathfrak{g}(2)
$$

with parabolic subalgebra

$$
\mathfrak{q}=\mathfrak{g}(0) \oplus \mathfrak{g}(1) \oplus \mathfrak{g}(2) .
$$

Here we have $\mathfrak{l}=\mathfrak{g}(0)$ and $\mathfrak{n}=\mathfrak{g}(1) \oplus \mathfrak{g}(2)$. The subalgebra $\overline{\mathfrak{n}}$, the opposite of $\mathfrak{n}$, is given by

$$
\overline{\mathfrak{n}}=\mathfrak{g}(-1) \oplus \mathfrak{g}(-2) .
$$

Let $\mathfrak{l}=\mathfrak{z}(\mathfrak{l}) \oplus[\mathfrak{l}, \mathfrak{l}]$ be the decomposition of $\mathfrak{l}$ that corresponds to $L=Z(L)^{\circ} L_{s s}$ with $Z(L)^{\circ}$ the identity component of the center of $L$ and $L_{s s}$ the semisimple part of $L$. We say that a weight $\nu \in \mathfrak{h}^{*}$ is a highest weight of a finite-dimensional $L$ module $V$ if $\left.\nu\right|_{\mathfrak{h}_{s s}}$ is a highest weight of $V$ as an $L_{s s}$-module, where $\mathfrak{h}_{s s}=\mathfrak{h} \cap[\mathfrak{l}, \mathfrak{l}]$. A lowest weight of a finite-dimensional $L$-module is similarly defined.

Proposition 4.5. Let $\mathfrak{q}=\mathfrak{g}(0) \oplus \mathfrak{g}(1) \oplus \mathfrak{g}(2)$ be the maximal parabolic of quasiHeisenberg type determined by $\alpha_{\mathfrak{q}}$.

(1) The subspace $\mathfrak{g}(1)$ is the irreducible $L$-module with lowest weight $\alpha_{\mathfrak{q}}$.

(2) The subspace $\mathfrak{g}(2)$ is the irreducible L-module with highest weight $\gamma$.

(3) We have $\mathfrak{z}(\mathfrak{n})=\mathfrak{g}(2)$.

Proof. Observe that, as $\operatorname{Ad}(L)$ preserves $\mathfrak{g}(j)$, to prove assertions (1) and (2), it suffices to consider $\mathfrak{g}(1)$ and $\mathfrak{g}(2)$ as $\mathfrak{l}$-modules. For assertion (1), the l-irreducibility of $\mathfrak{g}(1)$ just follows from a well-known fact that, for $\mathfrak{q}=\mathfrak{g}(0) \oplus \bigoplus \mathfrak{g}(j)_{j>0}$ with 
$\mathfrak{g}(1) \neq 0, \mathfrak{g}(1)$ is $\mathfrak{g}(0)$-irreducible if and only if $\mathfrak{q}$ is a maximal parabolic subalgebra. The lowest weight of $\mathfrak{g}(1)$ follows from Corollary 10.2A of [10]. For assertion (2), it is clear that $\mathcal{U}(\mathfrak{g}(0)) X_{\gamma} \subset \mathfrak{g}(2)$. On the other hand, as $\overline{\mathfrak{n}}=\mathfrak{g}(-1) \oplus \mathfrak{g}(-2)$, it follows that $\mathcal{U}(\overline{\mathfrak{n}}) \mathfrak{g}(2) \subset \bigoplus_{j=-2}^{1} \mathfrak{g}(j)$. As $\mathfrak{g}=\bigoplus_{j=-2}^{2} \mathfrak{g}(j)=\mathcal{U}(\overline{\mathfrak{n}})\left(\mathcal{U}(\mathfrak{g}(0)) X_{\gamma}\right)$, this shows that $\mathcal{U}(\mathfrak{g}(0)) X_{\gamma} \supset \mathfrak{g}(2)$. To prove assertion (3), since $\mathfrak{g}(2) \subset \mathfrak{z}(\mathfrak{n})$, it suffices to show the other inclusion. If $X \in \mathfrak{z}(\mathfrak{n})$, then, as $\mathfrak{n}=\mathfrak{g}(1) \oplus \mathfrak{g}(2)$, there exist $X_{j} \in \mathfrak{g}(j)$ for $j=1,2$ so that $X=X_{1}+X_{2}$. Since $X, X_{2} \in \mathfrak{z}(\mathfrak{n})$, for any $Y \in \mathfrak{n}$, we have

$$
\left[Y, X_{1}\right]=\left[Y, X_{1}\right]+\left[Y, X_{2}\right]=[Y, X]=0 .
$$

Thus $X_{1} \in \mathfrak{z}(\mathfrak{n}) \cap \mathfrak{g}(1)$. Now observe that assertion (1) implies that $\mathfrak{z}(\mathfrak{n}) \cap \mathfrak{g}(1)=\{0\}$. Thus $X_{1}=0$ and so $X=X_{2} \in \mathfrak{g}(2)$.

Now, since $\mathfrak{l}=\mathfrak{g}(0), \mathfrak{g}(2)=\mathfrak{z}(\mathfrak{n})$ and $\mathfrak{g}(-2)=\mathfrak{z}(\overline{\mathfrak{n}})$, we write the 2-grading $\mathfrak{g}=\bigoplus_{j=-2}^{2} \mathfrak{g}(j)$ as

$$
\mathfrak{g}=\mathfrak{z}(\overline{\mathfrak{n}}) \oplus \mathfrak{g}(-1) \oplus \mathfrak{l} \oplus \mathfrak{g}(1) \oplus \mathfrak{z}(\mathfrak{n})
$$

with parabolic subalgebra

$$
\mathfrak{q}=\mathfrak{l} \oplus \mathfrak{g}(1) \oplus \mathfrak{z}(\mathfrak{n}) .
$$

4.3. The simple ideals $\mathfrak{l}_{\gamma}$ and $\mathfrak{l}_{n_{\gamma}}$. We next observe the structure of the Levi subalgebra $\mathfrak{l}=\mathfrak{z}(\mathfrak{l}) \oplus[\mathfrak{l}, \mathfrak{l}]$. The structure of $\mathfrak{l}$ will play a role in Section 6, when we decompose $\mathfrak{l} \otimes \mathfrak{z}(\mathfrak{n})$ into irreducible $L$-submodules.

We start with the center $\mathfrak{z}(\mathfrak{l})$. The center $\mathfrak{z}(\mathfrak{l})$ is of the form $\mathfrak{z}(\mathfrak{l})=\bigcap_{\alpha \in \Pi(\mathfrak{l})} \operatorname{ker}(\alpha)$. Since $\mathfrak{g}$ has rank greater than one and since $\Pi(\mathfrak{l})=\Pi \backslash\left\{\alpha_{\mathfrak{q}}\right\}$, the center $\mathfrak{z}(\mathfrak{l})$ is nonzero and one-dimensional. It is clear from (4.4) that $H_{\mathfrak{q}}$ is an element of $\mathfrak{z}(\mathfrak{l})$. Therefore we have $\mathfrak{z}(\mathfrak{l})=\mathbb{C} H_{\mathfrak{q}}$.

Next we consider the structure of $[\mathfrak{l}, \mathfrak{l}]$. Observe that if $\mathfrak{g}$ is not of type $A_{n}$, then there is exactly one simple root that is not orthogonal to $\gamma$. Let $\alpha_{\gamma}$ denote the unique simple root. It is easy to see that $\mathfrak{q}_{\left\{\alpha_{\gamma}\right\}}$ is the parabolic subalgebra of the Heisenberg type of $\mathfrak{g}$, that is, the parabolic subalgebra with $\operatorname{dim}([\mathfrak{n}, \mathfrak{n}])=1$. Hence, if $\mathfrak{q}_{\left\{\alpha_{\mathfrak{q}}\right\}}$ is a maximal parabolic subalgebra of quasi-Heisenberg type, then $\alpha_{\gamma} \in \Pi(\mathfrak{l})=\Pi \backslash\left\{\alpha_{\mathfrak{q}}\right\}$. If we delete the node corresponding to $\alpha_{\mathfrak{q}}$, then we obtain one, two, or three subgraphs with one subgraph containing $\alpha_{\gamma}$. This implies that the subalgebra $[\mathfrak{l}, \mathfrak{l}]$ is either simple or the direct sum of two or three simple ideals with only one simple ideal containing the root space $\mathfrak{g}_{\alpha_{\gamma}}$ for $\alpha_{\gamma}$. The three subgraphs occur only when $\mathfrak{q}$ is of type $D_{n}(n-2)$. So, if $\mathfrak{q}$ is not of type $D_{n}(n-2)$, then there are at most two subgraphs. In this case we denote by $\mathfrak{l}_{\gamma}\left(\right.$ resp. $\left.\mathfrak{l}_{n \gamma}\right)$ the simple ideal of $\mathfrak{l}$ whose subgraph in the deleted Dynkin diagram contains (resp. does not contain) the node for $\alpha_{\gamma}$. Thus the Levi subalgebra $\mathfrak{l}$ may decompose into

$$
\mathfrak{l}=\mathbb{C} H_{\mathfrak{q}} \oplus \mathfrak{l}_{\gamma} \oplus \mathfrak{l}_{n \gamma} .
$$

Then, for the rest of this section, we assume that $\mathfrak{q}$ is not of type $D_{n}(n-2)$, so that the Levi subalgebra $\mathfrak{l}$ can be expressed as (4.8). Recall from Definition 4.3 that if $\mathfrak{g}$ is of type $\mathcal{T}$, then we say that the parabolic subalgebra $\mathfrak{q}$ determined by $\alpha_{i} \in \Pi$ is of type $\mathcal{T}(i)$. Then the parabolic subalgebras $\mathfrak{q}$ under consideration are given as follows 1

$$
B_{n}(i)(3 \leq i \leq n), \quad C_{n}(i)(2 \leq i \leq n-1), \quad D_{n}(i)(3 \leq i \leq n-3),
$$

\footnotetext{
${ }^{1}$ The case that $\mathfrak{q}$ is of type $D_{n}(n-2)$ is handled in 22 .
} 
and

$$
E_{6}(3), E_{6}(5), E_{7}(2), E_{7}(6), E_{8}(1), F_{4}(4) .
$$

Note that, in type $A_{n}$, any maximal parabolic subalgebra is of abelian type, and also that, in type $G_{2}$, the two maximal parabolic subalgebras are of either 3 -step type or Heisenberg type.

Write $\Pi\left(\mathfrak{l}_{\gamma}\right)=\left\{\alpha \in \Pi \mid \alpha \in \Delta\left(\mathfrak{l}_{\gamma}\right)\right\}$ and $\Pi\left(\mathfrak{l}_{n \gamma}\right)=\left\{\alpha \in \Pi \mid \alpha \in \Delta\left(\mathfrak{l}_{n \gamma}\right)\right\}$. Example 4.11 below exhibits the subgraphs for $\mathfrak{l}_{\gamma}$ and $\mathfrak{l}_{n \gamma}$ of $\mathfrak{q}$ of type $B_{5}(3)$ with $\Pi\left(\mathfrak{l}_{\gamma}\right)$ and $\Pi\left(\mathfrak{l}_{n \gamma}\right)$. One can find those data in Appendix $\mathrm{A}$ for each maximal parabolic subalgebra in (4.9) or (4.10).

Example 4.11. Let $\mathfrak{q}$ be the parabolic subalgebra of type $B_{5}(3)$ with deleted Dynkin diagram

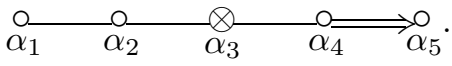

Observe that the unique simple root $\alpha_{\gamma}$ that is not orthogonal to the highest root $\gamma$ is $\alpha_{\gamma}=\alpha_{2}$. Therefore, the subgraph for $\mathfrak{l}_{\gamma}$ is

and that for $\mathfrak{l}_{n \gamma}$ is

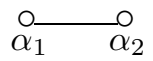

$$
\stackrel{\circ}{\alpha_{4}} \alpha_{5}
$$

with $\Pi\left(\mathfrak{l}_{\gamma}\right)=\left\{\alpha_{1}, \alpha_{2}\right\}$ and $\Pi\left(\mathfrak{l}_{n \gamma}\right)=\left\{\alpha_{4}, \alpha_{5}\right\}$.

Remark 4.12. As $\alpha_{\gamma}$ is the unique simple root that is not orthogonal to $\gamma$, we have $\left\langle\gamma, \alpha_{\gamma}\right\rangle>0$ and $\langle\gamma, \alpha\rangle=0$ for any other simple roots $\alpha$. In particular, $\langle\alpha, \gamma\rangle=0$ for all $\alpha \in \Pi\left(\mathfrak{l}_{n \gamma}\right)$.

4.4. The highest weights for $\mathfrak{l}_{\gamma}, \mathfrak{l}_{n_{\gamma}}, \mathfrak{g}(1)$, and $\mathfrak{z}(\mathfrak{n})$. For the rest of this section we summarize technical lemmas on the $L$-highest weights for $\mathfrak{l}_{\gamma}, \mathfrak{l}_{n \gamma}, \mathfrak{g}(1)$, and $\mathfrak{z}(\mathfrak{n})$. These technical facts will be used in later computations.

Proposition 4.5 shows that $\mathfrak{z}(\mathfrak{n})$ has highest weight $\gamma$, which is the highest root of $\mathfrak{g}$. We denote by $\xi_{\gamma}, \xi_{n \gamma}$, and $\mu$ the highest weights for $\mathfrak{l}_{\gamma}, \mathfrak{l}_{n \gamma}$, and $\mathfrak{g}(1)$, respectively. These highest weights are summarized in Appendix A for each of the parabolic subalgebras under consideration. We remark that all these highest weights are indeed roots in $\Delta^{+}$. Observe that the highest weights $\xi_{\gamma}$ and $\xi_{n \gamma}$ of $\mathfrak{l}_{\gamma}$ and $\mathfrak{l}_{n \gamma}$, respectively, are also the highest roots of $\mathfrak{l}_{\gamma}$ and $\mathfrak{l}_{n \gamma}$ as simple algebras; in particular, the multiplicities of $\alpha \in \Pi\left(\mathfrak{l}_{\gamma}\right)$ (resp. $\alpha \in \Pi\left(\mathfrak{l}_{n \gamma}\right)$ ) in $\xi_{\gamma}\left(\right.$ resp. $\left.\xi_{n \gamma}\right)$ are all strictly positive.

Lemma 4.13. If $\alpha_{\mathfrak{q}}$ is the simple root that determines $\mathfrak{q}=\mathfrak{l} \oplus \mathfrak{g}(1) \oplus \mathfrak{z}(\mathfrak{n})$, then $\xi_{\gamma}+\alpha_{\mathfrak{q}}$ and $\xi_{n \gamma}+\alpha_{\mathfrak{q}}$ are roots.

Proof. We only prove that $\xi_{\gamma}+\alpha_{\mathfrak{q}} \in \Delta$; the other assertion that $\xi_{n \gamma}+\alpha_{\mathfrak{q}} \in \Delta$ can be proven similarly. It suffices to show that $\left\langle\xi_{\gamma}, \alpha_{\mathfrak{q}}\right\rangle<0$, since both $\xi_{\gamma}$ and $\alpha_{\mathfrak{q}}$ are roots. For $\alpha \in \Pi$ we observe that $\left\langle\alpha, \alpha_{\mathfrak{q}}\right\rangle<0$ if $\alpha$ is adjacent to $\alpha_{\mathfrak{q}}$ in the Dynkin diagram and $\left\langle\alpha, \alpha_{\mathfrak{q}}\right\rangle=0$ otherwise. An observation on the deleted Dynkin diagrams shows that there exists a unique simple root $\alpha_{k}$ in $\Pi\left(\mathfrak{l}_{\gamma}\right)$ that is adjacent to $\alpha_{\mathfrak{q}}$. Since $\xi_{\gamma}$ is the highest root for $\mathfrak{l}_{\gamma}$ as a simple algebra, the multiplicity of $\alpha_{k}$ in $\xi_{\gamma}$ is strictly positive. Thus $\left\langle\xi_{\gamma}, \alpha_{\mathfrak{q}}\right\rangle<0$. 
Lemma 4.14. If $\xi_{\gamma}, \xi_{n \gamma}, \mu$, and $\gamma$ are the highest weights of $\mathfrak{l}_{\gamma}, \mathfrak{l}_{n \gamma}, \mathfrak{g}(1)$, and $\mathfrak{z}(\mathfrak{n})$, respectively, then the following hold:

(1) $\gamma-\xi_{\gamma} \in \Delta$, but $\gamma-\xi_{n \gamma} \notin \Delta$.

(2) $\gamma-\mu \in \Delta$.

(3) $\mu-\xi_{\gamma}, \mu-\xi_{n \gamma} \in \Delta$.

Proof. To prove $\gamma-\xi_{n \gamma} \notin \Delta$, observe that if $n$ and $m$ are the largest non-negative integers so that $\gamma-n \xi_{n \gamma} \in \Delta$ and $\gamma+m \xi_{n \gamma} \in \Delta$, respectively, then $\left\langle\gamma, \xi_{n \gamma}^{\vee}\right\rangle=n-m$. Since $\left\langle\gamma, \alpha^{\vee}\right\rangle=0$ for all $\alpha \in \Delta\left(\mathfrak{l}_{n \gamma}\right)$, we have $\left\langle\gamma, \xi_{n \gamma}^{\vee}\right\rangle=0$, and so $n=m$. As $\xi_{n \gamma} \in \Delta^{+}$and $\gamma$ is the highest root, $\gamma+\xi_{n \gamma} \notin \Delta$. Therefore, $n=m=0$, which concludes that $\gamma-\xi_{n \gamma}$ is not a root. To prove $\gamma-\xi_{\gamma} \in \Delta$, it suffices to show that $\left\langle\gamma, \xi_{\gamma}\right\rangle>0$, since both $\gamma$ and $\xi_{\gamma}$ are roots. Write $\xi_{\gamma}$ in terms of simple roots in $\Pi\left(\mathfrak{l}_{\gamma}\right)$. Observe that each $\alpha \in \Pi\left(\mathfrak{l}_{\gamma}\right)$ has positive multiplicity $m_{\alpha}$ in $\xi_{\gamma}$. As $\gamma$ is orthogonal to $\alpha$ for any $\alpha \in \Pi\left(\mathfrak{l}_{\gamma}\right) \backslash\left\{\alpha_{\gamma}\right\}$, we have $\left\langle\gamma, \xi_{\gamma}\right\rangle=m_{\alpha_{\gamma}}\left\langle\gamma, \alpha_{\gamma}\right\rangle>0$. Assertions (2) and (3) can be shown similarly.

The following technical lemma will simplify later arguments concerning the long roots. When $\mathfrak{g}$ is simply laced, we regard any root as a long root.

Lemma 4.15. Suppose that $\alpha \in \Delta$ is a long root. For any $\beta \in \Delta$, the following hold:

(1) If $\beta-\alpha \in \Delta$, then $\left\langle\beta, \alpha^{\vee}\right\rangle=1$.

(2) If $\beta+\alpha \in \Delta$, then $\left\langle\beta, \alpha^{\vee}\right\rangle=-1$.

(3) If $\beta \pm \alpha \in \Delta$, then $\beta \mp \alpha \notin \Delta$.

(4) $\beta \pm 2 \alpha \notin \Delta$.

Proof. These simply follow from the standard arguments using the structure theory of Lie algebras.

Lemma 4.16. If $\xi_{\gamma}, \xi_{n \gamma}, \mu$, and $\gamma$ are the highest weights of $\mathfrak{l}_{\gamma}, \mathfrak{l}_{n \gamma}, \mathfrak{g}(1)$, and $\mathfrak{z}(\mathfrak{n})$, respectively, then the following hold:

(1) $\gamma-\mu+\xi_{n \gamma} \in \Delta$.

(2) $\gamma-\mu-\xi_{n \gamma} \notin \Delta$.

(3) If $\xi_{\gamma}$ is a long root, then $\gamma-\mu \pm \xi_{\gamma} \notin \Delta$.

Proof. Lemma 4.14 shows that $\gamma-\mu \in \Delta$. Then in order to prove (1), it is enough to show that $\left\langle\xi_{n \gamma}, \gamma-\mu\right\rangle<0$. It follows from Remark 4.12 that $\left\langle\xi_{n \gamma}, \gamma\right\rangle=0$. On the other hand, we have $\left\langle\xi_{n \gamma}, \mu\right\rangle>0$ by the proof for (3) of Lemma 4.14. Therefore,

$$
\left\langle\xi_{n \gamma}, \gamma-\mu\right\rangle=\left\langle\xi_{n \gamma}, \gamma\right\rangle-\left\langle\xi_{n \gamma}, \mu\right\rangle<0 .
$$

When $\xi_{n \gamma}$ is a long root of $\mathfrak{g}$, assertion (2) follows from (1) and Lemma 4.15. The data in Appendix $\mathrm{A}$ shows that $\xi_{n \gamma}$ is a long root unless $\mathfrak{q}$ is of type $B_{n}(n-1)$. If $\mathfrak{q}$ is of type $B_{n}(n-1)$, then we have $\gamma=\varepsilon_{1}+\varepsilon_{2}, \mu=\varepsilon_{1}+\varepsilon_{n}$, and $\xi_{n \gamma}=\varepsilon_{n}$. Thus $\gamma-\mu-\xi_{n \gamma} \notin \Delta$.

To show (3), observe that, by Lemma 4.14, we have $\gamma-\xi_{\gamma}, \mu-\xi_{\gamma} \in \Delta$. Since $\xi_{\gamma}$ is assumed to be a long root, it follows from Lemma 4.15 that $\left\langle\gamma, \xi_{\gamma}^{\vee}\right\rangle=\left\langle\mu, \xi_{\gamma}^{\vee}\right\rangle=1$. Therefore $\left\langle\gamma-\mu, \xi_{\gamma}^{\vee}\right\rangle=0$, which forces that

$$
\left\|\gamma-\mu \pm \xi_{\gamma}\right\|^{2}=\|\gamma-\mu\|^{2}+\left\|\xi_{\gamma}\right\|^{2} .
$$

Since $\gamma-\mu$ is a root, we have $\|\gamma-\mu\| \neq 0$. As $\xi_{\gamma}$ is assumed to be a long root, (4.17) implies that $(\gamma-\mu) \pm \xi_{\gamma} \notin \Delta$. 
Remark 4.18. Direct observation shows that $\xi_{\gamma}$ is a long root, unless $\mathfrak{q}$ is of type $C_{n}(i)$. If $\mathfrak{q}$ is of type $C_{n}(i)$, then the data in Appendix $\mathrm{A}$ shows $\gamma=2 \varepsilon_{1}, \mu=$ $\varepsilon_{1}+\varepsilon_{i+1}$, and $\xi_{\gamma}=\varepsilon_{1}-\varepsilon_{i}$. Thus $\gamma-\mu+\xi_{\gamma} \notin \Delta$, but $\gamma-\mu-\xi_{\gamma} \in \Delta$.

\section{The $\Omega_{1}$ SYSTEM}

The aim of this section is to determine the complex parameter $s_{1} \in \mathbb{C}$ for the line bundle $\mathcal{L}_{s}$ so that the $\Omega_{1}$ system of a maximal parabolic subalgebra $\mathfrak{q}$ of quasiHeisenberg type is conformally invariant on $\mathcal{L}_{s_{1}}$. To do so, it is essential to set up convenient normalizations.

If $\alpha, \beta \in \Delta$, then define

$$
\begin{aligned}
p_{\alpha, \beta} & =\max \left\{j \in \mathbb{Z}_{\geq 0} \mid \beta-j \alpha \in \Delta\right\} \text { and } \\
q_{\alpha, \beta} & =\max \left\{j \in \mathbb{Z}_{\geq 0} \mid \beta+j \alpha \in \Delta\right\} .
\end{aligned}
$$

In particular, we have

$$
\left\langle\beta, \alpha^{\vee}\right\rangle=p_{\alpha, \beta}-q_{\alpha, \beta} .
$$

It is known that we can choose $X_{\alpha} \in \mathfrak{g}_{\alpha}$ and $H_{\alpha} \in \mathfrak{h}$ for each $\alpha \in \Delta$ in such a way that the following conditions hold (see for instance [8, Sections III.4 and III.5]). The reader may want to notice that our normalizations are different from those used in [1].

(H1) For each $\alpha \in \Delta^{+},\left\{X_{\alpha}, X_{-\alpha}, H_{\alpha}\right\}$ is an $\mathfrak{s l}(2)$-triple; in particular, we have $\left[X_{\alpha}, X_{-\alpha}\right]=H_{\alpha}$.

(H2) For each $\alpha, \beta \in \Delta^{+},\left[H_{\alpha}, X_{\beta}\right]=\beta\left(H_{\alpha}\right) X_{\beta}$.

(H3) For $\alpha \in \Delta$ we have $\kappa\left(X_{\alpha}, X_{-\alpha}\right)=1$.

(H4) For $\alpha, \beta \in \Delta$ we have $\beta\left(H_{\alpha}\right)=\langle\alpha, \beta\rangle$.

(H5) For $\alpha, \beta \in \Delta$ with $\alpha+\beta \neq 0$, there is a constant $N_{\alpha, \beta}$ so that

$$
\begin{aligned}
{\left[X_{\alpha}, X_{\beta}\right] } & =N_{\alpha, \beta} X_{\alpha+\beta} & & \text { if } \alpha+\beta \in \Delta, \\
N_{\alpha, \beta} & =0 & & \text { if } \alpha+\beta \notin \Delta .
\end{aligned}
$$

(H6) If $\alpha_{1}, \alpha_{2}, \alpha_{3} \in \Delta^{+}$with $\alpha_{1}+\alpha_{2}+\alpha_{3}=0$, then

$$
N_{\alpha_{1}, \alpha_{2}}=N_{\alpha_{2}, \alpha_{3}}=N_{\alpha_{3}, \alpha_{1}} \text {. }
$$

(H7) If $\alpha, \beta \in \Delta$ and $\alpha+\beta \in \Delta$, then

$$
N_{\alpha, \beta} N_{-\alpha,-\beta}=-\frac{q_{\alpha, \beta}\left(1+p_{\alpha, \beta}\right)}{2}\|\alpha\|^{2} .
$$

In particular, $N_{\alpha, \beta}$ is non-zero if $\alpha+\beta \in \Delta$.

We call the constants $N_{\alpha, \beta}$ structure constants. Observe that, by the normalization (H3), for all $\alpha \in \Delta$, the vector $X_{-\alpha}$ is the dual vector for $X_{\alpha}$ with respect to the Killing form. Therefore, in this normalization, the element $\omega=$ $\sum_{\gamma_{j} \in \Delta(\mathfrak{z}(\mathfrak{n}))} X_{\gamma_{j}}^{*} \otimes X_{\gamma_{j}} \in \mathfrak{z}(\overline{\mathfrak{n}}) \otimes \mathfrak{z}(\mathfrak{n})$ is

$$
\omega=\sum_{\gamma_{j} \in \Delta(\mathfrak{z}(\mathfrak{n})} X_{-\gamma_{j}} \otimes X_{\gamma_{j}}
$$

with $\left\{X_{-\alpha}, H_{\alpha}, X_{\alpha}\right\}$ an $\mathfrak{s l}(2)$-triple. (See Definition 3.1 for $\omega$.)

As we have observed in Subsection 3.1, we use the covariant map $\tau_{1}$ and the associated $L$-intertwining operators $\left.\tilde{\tau}_{1}\right|_{V^{*}}$, where $V^{*}$ are irreducible constituents of 
$\mathfrak{g}(-1)^{*} \otimes \mathfrak{g}(2)^{*}=\mathfrak{g}(-1)^{*} \otimes \mathfrak{z}(\mathfrak{n})^{*}$. By Definition 3.1. the covariant map $\tau_{1}$ is given by

$$
\begin{aligned}
\tau_{1}: \mathfrak{g}(1) & \rightarrow \mathfrak{g}(-1) \otimes \mathfrak{z}(\mathfrak{n}), \\
X & \mapsto(\operatorname{ad}(X) \otimes \mathrm{Id}) \omega
\end{aligned}
$$

with $\omega=\sum_{\gamma_{j} \in \Delta(\mathfrak{z}(\mathfrak{n}))} X_{-\gamma_{j}} \otimes X_{\gamma_{j}}$. It is clear that $\tau_{1}$ is not identically zero. Indeed, if $X=X_{\mu}$ with $\mu$ the highest weight for $\mathfrak{g}(1)$, then

$$
\tau_{1}\left(X_{\mu}\right)=\left(\operatorname{ad}\left(X_{\mu}\right) \otimes \mathrm{Id}\right) \omega=\sum_{\Delta_{\mu}(\mathfrak{z}(\mathfrak{n}))} N_{\mu,-\gamma_{j}} X_{\mu-\gamma_{j}} \otimes X_{\gamma_{j}}
$$

with $\Delta_{\mu}(\mathfrak{z}(\mathfrak{n}))=\left\{\gamma_{j} \in \Delta(\mathfrak{z}(\mathfrak{n})) \mid \mu-\gamma_{j} \in \Delta\right\}$. By Lemma 4.14, we have $\mu-\gamma \in \Delta$ with $\gamma$ the highest weight for $\mathfrak{z}(\mathfrak{n})$, so $\Delta_{\mu}(\mathfrak{z}(\mathfrak{n})) \neq \emptyset$. Since the vectors $X_{\mu-\gamma_{j}} \otimes X_{\gamma_{j}}$ for $\gamma_{j} \in \Delta_{\mu}(\mathfrak{z}(\mathfrak{n}))$ are linearly independent, we have $\tau_{1}\left(X_{\mu}\right) \neq 0$.

For each irreducible constituent $V^{*}$ of $\mathfrak{g}(-1)^{*} \otimes \mathfrak{z}(\mathfrak{n})^{*}$, there exists an associated $L$-intertwining operator $\left.\tilde{\tau}_{1}\right|_{V^{*}} \in \operatorname{Hom}_{L}\left(V^{*}, \mathcal{P}^{1}(\mathfrak{g}(1))\right)$ so that, for all $Y^{*} \in V^{*}$,

$$
\left.\tilde{\tau}_{1}\right|_{V^{*}}\left(Y^{*}\right)(X)=Y^{*}\left(\tau_{1}(X)\right) .
$$

Observe that the duality for $V^{*}$ is defined with respect to the Killing form $\kappa$. Moreover, via the Killing form $\kappa$, we have $\mathfrak{g}(-1)^{*} \otimes \mathfrak{z}(\mathfrak{n})^{*} \cong \mathfrak{g}(1) \otimes \mathfrak{z}(\overline{\mathfrak{n}})$. Thus, if $Y^{*}=X_{\alpha} \otimes X_{-\gamma_{t}}$ with $\alpha \in \Delta(\mathfrak{g}(1))$ and $\gamma_{t} \in \Delta(\mathfrak{z}(\mathfrak{n}))$, then $Y^{*}\left(\tau_{1}(X)\right)$ is given by

$$
Y^{*}\left(\tau_{1}(X)\right)=\sum_{\gamma_{j} \in \Delta(\mathfrak{z}(\mathfrak{n}))} \kappa\left(X_{\alpha}, \operatorname{ad}(X) X_{-\gamma_{j}}\right) \kappa\left(X_{-\gamma_{t}}, X_{\gamma_{j}}\right),
$$

as $\tau_{1}(X)=\sum_{\gamma_{j} \in \Delta(\mathfrak{z}(\mathfrak{n}))} \operatorname{ad}(X) X_{-\gamma_{j}} \otimes X_{\gamma_{j}}$.

Now we wish to determine all the irreducible constituents $V^{*}$ of $\mathfrak{g}(1) \otimes \mathfrak{z}(\overline{\mathfrak{n}})$ so that $\left.\tilde{\tau}_{1}\right|_{V^{*}}$ are not identically zero. Observe that $\mathcal{P}^{1}(\mathfrak{g}(1)) \cong \operatorname{Sym}^{1}(\mathfrak{g}(-1))=\mathfrak{g}(-1)$ and that $\mathfrak{g}(-1)$ is an irreducible $L$-module, as $\mathfrak{q}$ is a maximal parabolic subalgebra. Thus, if $\left.\tilde{\tau}_{1}\right|_{V^{*}}$ is not identically zero, then $V^{*} \cong \mathfrak{g}(-1)$. Proposition 5.4 below shows that the converse also holds.

Proposition 5.4. Let $V^{*}$ be an irreducible constituent of $\mathfrak{g}(1) \otimes \mathfrak{z}(\overline{\mathfrak{n}})$. Then $\left.\tilde{\tau}_{1}\right|_{V^{*}}$ is not identically zero if and only if $V^{*} \cong \mathfrak{g}(-1)$.

Proof. First observe that $\mathfrak{g}(-1)$ is an irreducible constituent of $\mathfrak{g}(1) \otimes \mathfrak{z}(\overline{\mathfrak{n}})$. Indeed, since $\tau_{1}$ is linear, we have $\tau_{1}(\mathfrak{g}(1)) \cong \mathfrak{g}(1)$ as an $L$-module; in particular, $\mathfrak{g}(1)$ is an irreducible constituent of $\mathfrak{g}(-1) \otimes \mathfrak{z}(\mathfrak{n})$. Therefore $\mathfrak{g}(-1) \cong \mathfrak{g}(1)^{*}$ is an irreducible constituent of $\mathfrak{g}(1) \otimes \mathfrak{z}(\overline{\mathfrak{n}}) \cong(\mathfrak{g}(-1) \otimes \mathfrak{z}(\mathfrak{n}))^{*}$.

To prove that $\left.\tilde{\tau}_{1}\right|_{\mathfrak{g}(-1)}$ is a non-zero map, it suffices to show that $\left.\tilde{\tau}_{1}\right|_{\mathfrak{g}(-1)}\left(Y^{*}\right) \neq 0$ for some $Y^{*} \in \mathfrak{g}(-1) \subset \mathfrak{g}(1) \otimes \mathfrak{z}(\overline{\mathfrak{n}})$. To do so, consider a map

$$
\begin{aligned}
\bar{\tau}_{1}: \mathfrak{g}(-1) & \rightarrow \mathfrak{g}(1) \otimes \mathfrak{z}(\overline{\mathfrak{n}}), \\
\bar{X} & \mapsto(\operatorname{ad}(\bar{X}) \otimes \operatorname{Id}) \bar{\omega}
\end{aligned}
$$

with $\bar{\omega}=\sum_{\gamma_{t} \in \Delta(\mathfrak{z}(\mathfrak{n}))} X_{\gamma_{t}} \otimes X_{-\gamma_{t}}$. This is a non-zero $L$-intertwining operator. Thus $\bar{\tau}_{1}(\mathfrak{g}(-1)) \cong \mathfrak{g}(-1)$ as an $L$-module, and $\bar{\tau}_{1}\left(X_{-\alpha}\right)$ is a weight vector with weight $-\alpha$ for all $\alpha \in \Delta(\mathfrak{g}(1))$. As $\mathfrak{g}(1)$ has highest weight $\mu$, the lowest weight for $\mathfrak{g}(-1)$ is $-\mu$.

Now we set

$$
c_{\mu}=\sum_{\gamma_{t} \in \Delta_{\mu}(\mathfrak{z}(\mathfrak{n}))} N_{-\mu, \gamma_{t}} N_{\mu,-\gamma_{t}}
$$


with $\Delta_{\mu}(\mathfrak{z}(\mathfrak{n}))=\left\{\gamma_{t} \in \Delta(\mathfrak{z}(\mathfrak{n})) \mid \gamma_{t}-\mu \in \Delta\right\}$. By Lemma 4.14, it follows that $\gamma-\mu \in \Delta$; in particular, $\Delta_{\mu}(\mathfrak{z}(\mathfrak{n})) \neq \emptyset$. The normalization (H7) shows that $N_{-\mu, \gamma_{t}} N_{\mu,-\gamma_{t}}<0$ for all $\gamma_{t} \in \Delta_{\mu}(\mathfrak{z}(\mathfrak{n}))$. Therefore $c_{\mu} \neq 0$. Then define $Y_{l}^{*} \in \mathfrak{g}(-1)$ by means of

$$
Y_{l}^{*}=\frac{1}{c_{\mu}} \bar{\tau}_{1}\left(X_{-\mu}\right)=\frac{1}{c_{\mu}} \sum_{\gamma_{t} \in \Delta_{\mu}(\mathfrak{z}(\mathfrak{n}))} N_{-\mu, \gamma_{t}} X_{\gamma_{t}-\mu} \otimes X_{-\gamma_{t}} .
$$

We claim that $\left.\tilde{\tau}_{1}\right|_{\mathfrak{g}(-1)}\left(Y_{l}^{*}\right)(X) \neq 0$. By (5.3), the polynomial $\left.\tilde{\tau}_{1}\right|_{\mathfrak{g}(-1)}\left(Y_{l}^{*}\right)(X)$ is

$$
\begin{aligned}
\left.\tilde{\tau}_{1}\right|_{\mathfrak{g}(-1)}\left(Y_{l}^{*}\right)(X) & =Y_{l}^{*}\left(\tau_{1}(X)\right) \\
& =\frac{1}{c_{\mu}} \sum_{\substack{\left.\gamma_{t} \in \Delta_{\mu}(\mathfrak{z}(\mathfrak{n})) \\
\gamma_{j} \in \Delta(\mathfrak{z})\right)}} N_{-\mu, \gamma_{t}} \kappa\left(X_{\gamma_{t}-\mu}, \operatorname{ad}(X) X_{-\gamma_{j}}\right) \kappa\left(X_{-\gamma_{t}}, X_{\gamma_{j}}\right) \\
& =\frac{1}{c_{\mu}} \sum_{\gamma_{t} \in \Delta_{\mu}(\mathfrak{z}(\mathfrak{n}))} N_{-\mu, \gamma_{t}} \kappa\left(X_{\gamma_{t}-\mu}, \operatorname{ad}(X) X_{-\gamma_{t}}\right) .
\end{aligned}
$$

Write $X=\sum_{\alpha \in \Delta(\mathfrak{g}(1))} \eta_{\alpha} X_{\alpha}$, where $\eta_{\alpha} \in \mathfrak{n}^{*}$ is the coordinate dual to $X_{\alpha}$ with respect to the Killing form $\kappa$. Then,

$$
\begin{aligned}
\left.\tilde{\tau}_{1}\right|_{\mathfrak{g}(-1)}\left(Y_{l}^{*}\right)(X) & =\frac{1}{c_{\mu}} \sum_{\gamma_{t} \in \Delta_{\mu}(\mathfrak{z}(\mathfrak{n}))} N_{-\mu, \gamma_{t}} \kappa\left(X_{\gamma_{t}-\mu}, \operatorname{ad}(X) X_{-\gamma_{t}}\right) \\
& =\frac{1}{c_{\mu}} \sum_{\substack{\alpha \in \Delta(\mathfrak{g}(1)) \\
\gamma_{t} \in \Delta_{\mu}(\mathfrak{z}(\mathfrak{n}))}} N_{-\mu, \gamma_{t}} \eta_{\alpha} \kappa\left(X_{\gamma_{t}-\mu}, \operatorname{ad}\left(X_{\alpha}\right) X_{-\gamma_{t}}\right) \\
& =\frac{1}{c_{\mu}} \sum_{\gamma_{t} \in \Delta_{\mu}(\mathfrak{z}(\mathfrak{n}))} N_{-\mu, \gamma_{t}} N_{\mu,-\gamma_{t}} \eta_{\mu} \\
& =\eta_{\mu} \\
& =\kappa\left(X, X_{-\mu}\right) .
\end{aligned}
$$

Hence $\left.\tilde{\tau}_{1}\right|_{\mathfrak{g}(-1)}\left(Y_{l}^{*}\right)(X) \neq 0$.

Since only $\mathfrak{g}(-1)$ contributes to the construction of the $\Omega_{1}$ systems, we simply refer to the $\Omega_{1}$ system as the $\left.\Omega_{1}\right|_{\mathfrak{g}(-1)}$ system. As we observed in Subsection 3.1, the operator $\left.\Omega_{1}\right|_{\mathfrak{g}(-1)}: \mathfrak{g}(-1) \rightarrow \mathbb{D}\left(\mathcal{L}_{s}\right)^{\overline{\mathfrak{n}}}$ is obtained via the composition of maps

$$
\mathfrak{g}(-1) \stackrel{\left.\tilde{\tau}_{1}\right|_{\mathfrak{g}(-1)}}{\rightarrow} \mathcal{P}^{1}(\mathfrak{g}(1)) \rightarrow \mathfrak{g}(-1) \stackrel{\sigma}{\rightarrow} \mathcal{U}(\overline{\mathfrak{n}}) \stackrel{R}{\rightarrow} \mathbb{D}\left(\mathcal{L}_{s}\right)^{\overline{\mathfrak{n}}}
$$

By (5.5), we have $\left.\tilde{\tau}_{1}\right|_{\mathfrak{g}(-1)}\left(Y_{l}^{*}\right)(X)=\kappa\left(X, X_{-\mu}\right)$. Therefore,

$$
\Omega_{1}\left(Y_{l}^{*}\right)=R\left(X_{-\mu}\right) .
$$

Now, for all $\alpha \in \Delta(\mathfrak{g}(1))$, set

$$
Y_{-\alpha}=\bar{\tau}_{1}\left(X_{-\alpha}\right) \text {. }
$$

Then, as $Y_{l}^{*}=\left(1 / c_{\mu}\right) \bar{\tau}_{1}\left(X_{-\mu}\right)$, we have $\Omega_{1}\left(Y_{-\mu}\right)=c_{\mu} R\left(X_{-\mu}\right)$. Since both $\left.\Omega_{1}\right|_{\mathfrak{g}(-1)}$ and $\bar{\tau}_{1}$ are $L_{0}$-intertwining operators and since $\mathfrak{g}(-1)=\mathcal{U}(\mathfrak{l}) X_{-\mu}$, for any $\alpha \in$ $\Delta(\mathfrak{g}(1))$, we obtain

$$
\Omega_{1}\left(Y_{-\alpha}\right)=c_{\alpha} R\left(X_{-\alpha}\right)
$$


with some constant $c_{\alpha}$. Thus, if $\Delta(\mathfrak{g}(1))=\left\{\alpha_{1}, \ldots, \alpha_{m}\right\}$, then the $\Omega_{1}$ system is given by

$$
R\left(X_{-\alpha_{1}}\right), \ldots, R\left(X_{-\alpha_{m}}\right) .
$$

Theorem 5.7. Let $\mathfrak{g}$ be a complex simple Lie algebra, and let $\mathfrak{q}$ be a maximal parabolic subalgebra of quasi-Heisenberg type. Then the $\Omega_{1}$ system is conformally invariant on $\mathcal{L}_{s}$ if and only if $s=0$.

Proof. By Remark 3.8, we only need to show that condition (S2) in Definition 2.3 holds if and only if $s=0$. By Proposition 2.17 for any $Y \in \mathfrak{g}$ and any $f \in C^{\infty}\left(\bar{N}_{0}, \mathbb{C}_{\chi^{s}}\right)$, we have

$$
\begin{aligned}
& \left(\left[\pi_{s}(Y), R\left(X_{-\alpha_{j}}\right)\right] \bullet f\right)(\bar{n}) \\
& =\left(R\left(\left[\left(\operatorname{Ad}\left(\bar{n}^{-1}\right) Y\right)_{\mathfrak{q}}, X_{-\alpha_{j}}\right]_{\overline{\mathfrak{n}}}\right) \bullet f\right)(\bar{n})-s \lambda_{\mathfrak{q}}\left(\left[\operatorname{Ad}\left(\bar{n}^{-1}\right) Y, X_{-\alpha_{j}}\right]_{\mathfrak{q}}\right) f(\bar{n}) .
\end{aligned}
$$

Hence, condition (S2) holds if and only if $s=0$.

\section{Special Constituents of $\mathfrak{l} \otimes \mathfrak{z}(\mathfrak{n})$}

Our next goal is to construct the $\Omega_{2}$ systems and to find their special values. To do so, we need to detect the irreducible constituents $V^{*}$ of $\mathfrak{l}^{*} \otimes \mathfrak{z}(\mathfrak{n})^{*}$ so that $\left.\tilde{\tau}_{2}\right|_{V^{*}}$ is not identically zero. (See Subsection 3.1 for the general construction of the $\Omega_{k}$ systems.) In this section we shall show preliminary results to find such irreducible constituents.

6.1. Irreducible decomposition of $\mathfrak{l}_{\gamma} \otimes \mathfrak{z}(\mathfrak{n})$. We continue with $\mathfrak{q}=\mathfrak{l} \oplus \mathfrak{g}(1) \oplus \mathfrak{z}(\mathfrak{n})$ a maximal parabolic subalgebra of quasi-Heisenberg type listed in (4.9) or (4.10), and $Q=L N=N_{G}(\mathfrak{q})$. The Levi subgroup $L$ acts on $\mathfrak{l} \otimes \mathfrak{z}(\mathfrak{n}) \subset \mathfrak{g} \otimes \mathfrak{g}$ via the standard action on the tensor product induced by the adjoint representation on $\mathfrak{l}$ and $\mathfrak{z}(\mathfrak{n})$. As $L$ is complex reductive, this action is completely reducible. Since $\mathfrak{l}=\mathfrak{z}(\mathfrak{l}) \oplus \mathfrak{l}_{\gamma} \oplus \mathfrak{l}_{n \gamma}$ with $\mathfrak{z}(\mathfrak{l})=\mathbb{C} H_{\mathfrak{q}}$, we have

$$
\mathfrak{l} \otimes \mathfrak{z}(\mathfrak{n})=\left(\mathbb{C} H_{\mathfrak{q}} \otimes \mathfrak{z}(\mathfrak{n})\right) \oplus\left(\mathfrak{l}_{\gamma} \otimes \mathfrak{z}(\mathfrak{n})\right) \oplus\left(\mathfrak{l}_{n \gamma} \otimes \mathfrak{z}(\mathfrak{n})\right) .
$$

It is clear that $\mathbb{C} H_{\mathfrak{q}} \otimes \mathfrak{z}(\mathfrak{n}) \cong \mathfrak{z}(\mathfrak{n})=\mathfrak{g}(2)$ as an $L$-module. Thus, by Proposition 4.5, $\mathbb{C} H_{\mathfrak{q}} \otimes \mathfrak{z}(\mathfrak{n})$ is $L$-irreducible. It is also easy to show that $\mathfrak{l}_{n \gamma} \otimes \mathfrak{z}(\mathfrak{n})$ is $L$-irreducible. Let $L_{\gamma}\left(\right.$ resp. $\left.L_{n \gamma}\right)$ be the analytic subgroup of $L$ with Lie algebra $\mathfrak{l}_{\gamma}\left(\operatorname{resp} . \mathfrak{l}_{n \gamma}\right)$. As in Subsection 4.2, we call a weight $\nu$ for a finite-dimensional $L$-module $V$ a highest weight for $V$ if the restriction $\left.\nu\right|_{\mathfrak{h}_{s s}}$ onto $\mathfrak{h}_{s s}$ is a highest weight for $V$ as an $L_{s s}$-module.

Proposition 6.2. Suppose that $\mathfrak{l}_{n \gamma} \neq 0$. If $\xi_{n \gamma}$ and $\gamma$ are the highest weights of $\mathfrak{l}_{n \gamma}$ and $\mathfrak{z}(\mathfrak{n})$, respectively, then $\mathfrak{l}_{n \gamma} \otimes \mathfrak{z}(\mathfrak{n})$ is the irreducible L-module with highest weight $\xi_{n \gamma}+\gamma$.

Proof. First we observe that $L_{n \gamma}$ acts trivially on $\mathfrak{z}(\mathfrak{n})$. By Proposition 4.5, we have $\mathfrak{z}(\mathfrak{n})=\mathfrak{g}(2)=\mathcal{U}([\mathfrak{l}, \mathfrak{l}]) X_{\gamma}$. By the observation made in Remark 4.12, it follows that $\alpha \perp \gamma$ for all $\alpha \in \Delta\left(\mathfrak{l}_{n \gamma}\right)$. Thus $\mathfrak{z}(\mathfrak{n})=\mathcal{U}\left(\mathfrak{l}_{\gamma}\right) X_{\gamma}$. Hence $L_{n \gamma}$ acts trivially; in particular, the irreducible $L$-module $\mathfrak{z}(\mathfrak{n})$ is $L_{\gamma}$-irreducible. On the other hand, it is clear that $L_{\gamma}$ acts on $\mathfrak{l}_{n \gamma}$ trivially. Therefore the representation $\left(L, \operatorname{Ad} \otimes \operatorname{Ad}, \mathfrak{l}_{n \gamma} \otimes\right.$ $\mathfrak{z}(\mathfrak{n}))$ is equivalent to $\left(L_{\gamma} \times L_{n \gamma}, \operatorname{Ad} \hat{\otimes} \operatorname{Ad}, \mathfrak{l}_{n \gamma} \otimes \mathfrak{z}(\mathfrak{n})\right)$, where $\hat{\otimes}$ denotes the outer tensor product. Since $\mathfrak{l}_{n \gamma}$ and $\mathfrak{z}(\mathfrak{n})$ have highest weight $\xi_{n \gamma}$ and $\gamma$, respectively, the lemma follows. 
Now we focus on the irreducible decomposition of $\mathfrak{l}_{\gamma} \otimes \mathfrak{z}(\mathfrak{n})$. As noted in the proof for Proposition 6.2 the subgroup $L_{n \gamma}$ acts trivially on $\mathfrak{l}_{\gamma} \otimes \mathfrak{z}(\mathfrak{n})$. Hence we study $\mathfrak{l}_{\gamma} \otimes \mathfrak{z}(\mathfrak{n})$ as an $L_{\gamma}$-module. For $\lambda \in \mathfrak{h}^{*}$ with $\left\langle\lambda, \alpha^{\vee}\right\rangle \in \mathbb{Z}_{\geq 0}$ for all $\alpha \in \Pi\left(\mathfrak{l}_{\gamma}\right)$, we will denote by $V(\lambda)$ the irreducible constituent with highest weight $\left.\lambda\right|_{\mathfrak{h}_{\gamma}}$, where $\mathfrak{h}_{\gamma}=\mathfrak{h} \cap \mathfrak{l}_{\gamma}$. For classical algebras, we use the standard realization of the roots $\varepsilon_{i}$, the dual basis of the standard orthonormal basis for $\mathbb{R}^{n}$. For exceptional algebras the Bourbaki conventions are used to label the simple roots.

Theorem 6.3. The L-module $\mathfrak{l}_{\gamma} \otimes \mathfrak{z}(\mathfrak{n})$ is reducible. If $V(\lambda)$ denotes the irreducible representation of $L$ with highest weight $\left.\lambda\right|_{\mathfrak{h}_{\gamma}}$, then the irreducible decomposition of $\mathfrak{l}_{\gamma} \otimes \mathfrak{z}(\mathfrak{n})$ is given as follows:

(1) $B_{n}(i), 3 \leq i \leq n$ :

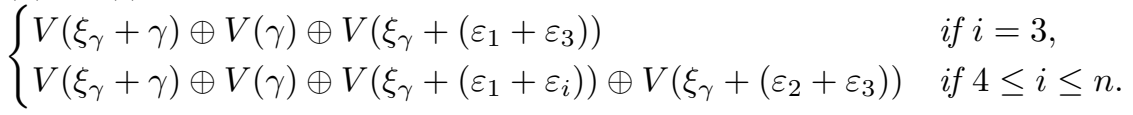

(2) $C_{n}(i), 2 \leq i \leq n-1$ :

$\begin{cases}V\left(\xi_{\gamma}+\gamma\right) \oplus V(\gamma) \oplus V\left(\xi_{\gamma}+2 \varepsilon_{2}\right) & \text { if } i=2, \\ V\left(\xi_{\gamma}+\gamma\right) \oplus V(\gamma) \oplus V\left(\xi_{\gamma}+\left(\varepsilon_{2}+\varepsilon_{i}\right)\right) \oplus V\left(\xi_{\gamma}+\left(\varepsilon_{1}+\varepsilon_{2}\right)\right) & \text { if } 3 \leq i \leq n-1\end{cases}$

(3) $D_{n}(i), 3 \leq i \leq n-3$ :

$\begin{cases}V\left(\xi_{\gamma}+\gamma\right) \oplus V(\gamma) \oplus V\left(\xi_{\gamma}+\left(\varepsilon_{1}+\varepsilon_{3}\right)\right) & \text { if } i=3, \\ V\left(\xi_{\gamma}+\gamma\right) \oplus V(\gamma) \oplus V\left(\xi_{\gamma}+\left(\varepsilon_{1}+\varepsilon_{i}\right)\right) \oplus V\left(\xi_{\gamma}+\left(\varepsilon_{2}+\varepsilon_{3}\right)\right) & \text { if } 4 \leq i \leq n-3 .\end{cases}$

(4) All exceptional cases $\left(E_{6}(3), E_{6}(5), E_{7}(2), E_{7}(6), E_{8}(1), F_{4}(4)\right)$ :

$$
V\left(\xi_{\gamma}+\gamma\right) \oplus V(\gamma) \oplus V\left(\xi_{\gamma}+\gamma_{0}\right),
$$

where $\gamma_{0}$ is the following root contributing to $\mathfrak{z}(\mathfrak{n})$ :

$$
\begin{aligned}
& E_{6}(3): \gamma_{0}=\alpha_{1}+\alpha_{2}+2 \alpha_{3}+3 \alpha_{4}+2 \alpha_{5}+\alpha_{6}, \\
& E_{6}(5): \gamma_{0}=\alpha_{1}+\alpha_{2}+2 \alpha_{3}+3 \alpha_{4}+2 \alpha_{5}+\alpha_{6}, \\
& E_{7}(2): \gamma_{0}=\alpha_{1}+2 \alpha_{2}+3 \alpha_{3}+4 \alpha_{4}+3 \alpha_{5}+2 \alpha_{6}+\alpha_{7}, \\
& E_{7}(6): \gamma_{0}=\alpha_{1}+2 \alpha_{2}+2 \alpha_{3}+4 \alpha_{4}+3 \alpha_{5}+2 \alpha_{6}+\alpha_{7}, \\
& E_{8}(1): \gamma_{0}=2 \alpha_{1}+3 \alpha_{2}+4 \alpha_{3}+6 \alpha_{4}+5 \alpha_{5}+4 \alpha_{6}+2 \alpha_{7}+\alpha_{8}, \\
& F_{4}(4): \gamma_{0}=\alpha_{1}+2 \alpha_{2}+4 \alpha_{3}+2 \alpha_{4} .
\end{aligned}
$$

Proof. To prove this theorem we just use the standard character formula due to Klimyk ([16, Corollary]) for $\mathfrak{l}_{\gamma} \otimes \mathfrak{z}(\mathfrak{n})$. For the details, see Chapter 5 of [20].

6.2. Special constituents. Given the irreducible constituent $V(\nu)$ in $\mathfrak{l} \otimes \mathfrak{z}(\mathfrak{n})$, we build the $L$-intertwining map

$$
\left.\tilde{\tau}_{2}\right|_{V(\nu)^{*}} \in \operatorname{Hom}_{L}\left(V(\nu)^{*}, \mathcal{P}^{2}(\mathfrak{g}(1))\right),
$$

with $V(\nu)^{*}$ the dual of $V(\nu)$ with respect to the Killing form $\kappa$. From $\left.\tilde{\tau}_{2}\right|_{V(\nu)^{*}}$, we construct the operator $\left.\Omega_{2}\right|_{V(\nu)^{*}}: V(\nu)^{*} \rightarrow \mathbb{D}\left(\mathcal{L}_{s}\right)^{\overline{\mathfrak{n}}}$. To do so, it is necessary to determine which irreducible constituents $V(\nu)$ have the property that $\left.\tilde{\tau}_{2}\right|_{V(\nu)^{*}} \neq 0$. Thus, next, by using the above decomposition results, we shall determine such irreducible constituents.

First we observe the vector space isomorphism $\mathcal{P}^{2}(\mathfrak{g}(1)) \cong \operatorname{Sym}^{2}(\mathfrak{g}(1))^{*}$. With the natural $L$-action on $\mathcal{P}^{2}(\mathfrak{g}(1))$ and $\operatorname{Sym}^{2}(\mathfrak{g}(1))^{*}$, this vector space isomorphism 
is $L$-equivariant. Thus, if $\left.\tilde{\tau}_{2}\right|_{V(\nu)^{*}}$ is a non-zero map, then $V(\nu)$ is an irreducible constituent of $\operatorname{Sym}^{2}(\mathfrak{g}(1)) \subset \mathfrak{g}(1) \otimes \mathfrak{g}(1)$; in particular, the weight $\nu$ is of the form $\nu=\mu+\epsilon$ for some $\epsilon \in \Delta(\mathfrak{g}(1))$, where $\mu$ is the highest weight of $\mathfrak{g}(1)$.

One can see from the decompositions in Theorem 6.3 that $V(\gamma)$ is an irreducible constituent of $\mathfrak{l} \otimes \mathfrak{z}(\mathfrak{n})$ for any $\mathfrak{q}$ under consideration. By Lemma 4.14, we have $\gamma=\mu+\epsilon$ for some $\epsilon \in \Delta(\mathfrak{g}(1))$. Now we claim that $\left.\tilde{\tau}_{2}\right|_{V(\gamma)^{*}}$ is identically zero. It is well known that

$$
\mathfrak{g}(1) \otimes \mathfrak{g}(1)=\operatorname{Sym}^{2}(\mathfrak{g}(1)) \oplus \wedge^{2}(\mathfrak{g}(1))
$$

as an $L$-module. Since each weight space for $\mathfrak{g}(1)$ is one-dimensional as weights for $\mathfrak{g}(1)$ are roots of $\mathfrak{g}$, the $L$-module decomposition (6.4) is multiplicity free.

Proposition 6.5. The L-module $V(\gamma)$ is an irreducible constituent of $\bigwedge^{2}(\mathfrak{g}(1))$.

Proof. Define a linear map $\varphi: \mathfrak{z}(\mathfrak{n}) \rightarrow \bigwedge^{2}(\mathfrak{g}(1))$ by means of

$$
\varphi(W)=\sum_{\beta \in \Delta(\mathfrak{g}(1))} \operatorname{ad}(W) X_{-\beta} \wedge X_{\beta} .
$$

By using an argument similar to that for Lemma 3.3. one can show that $\varphi$ is $L$ equivariant. Then, since $\mathfrak{z}(\mathfrak{n}) \cong V(\gamma)$ as an irreducible $L$-module, it suffices to show that $\varphi$ is a non-zero map. Write $\Delta_{\gamma}(\mathfrak{g}(1))=\{\beta \in \Delta(\mathfrak{g}(1)) \mid \gamma-\beta \in \Delta\}$. By Lemma 4.14, we have $\gamma-\mu \in \Delta$. Hence $\Delta_{\gamma}(\mathfrak{g}(1)) \neq \emptyset$. By writing $\beta^{\prime}=\gamma-\beta$ for $\beta \in \Delta_{\gamma}(\mathfrak{g}(1)), \varphi\left(X_{\gamma}\right)$ is given by

$$
\varphi\left(X_{\gamma}\right)=\sum_{\beta \in \Delta(\mathfrak{g}(1))} \operatorname{ad}\left(X_{\gamma}\right) X_{-\beta} \wedge X_{\beta}=\sum_{\beta \in \Delta_{\gamma}(\mathfrak{g}(1))} N_{\gamma,-\beta} X_{\beta^{\prime}} \wedge X_{\beta} .
$$

Observe that for each $\beta \in \Delta_{\gamma}(\mathfrak{g}(1))$, we have $\gamma-\beta \in \Delta_{\gamma}(\mathfrak{g}(1))$. Moreover, by the normalization (H6) of our normalizations in Section 5, it follows that $N_{\gamma,-\beta^{\prime}}=$ $-N_{\gamma,-\beta}$. Therefore,

$$
N_{\gamma,-\beta} X_{\beta^{\prime}} \wedge X_{\beta}+N_{\gamma,-\beta^{\prime}} X_{\beta} \wedge X_{\beta^{\prime}}=2 N_{\gamma,-\beta} X_{\beta^{\prime}} \wedge X_{\beta} .
$$

Since $N_{\gamma,-\beta} \neq 0$ for $\beta \in \Delta_{\gamma}(\mathfrak{g}(1))$, equation (6.6) is non-zero. On the other hand, if $\beta \in \Delta_{\gamma}(\mathfrak{g}(1))$ and $\eta \in \Delta_{\gamma}(\mathfrak{g}(1))$ so that $\eta \neq \beta, \beta^{\prime}$, then $X_{\beta^{\prime}} \wedge X_{\beta}$ and $X_{\eta} \wedge X_{\beta}$ are linearly independent. Hence, $\varphi\left(X_{\gamma}\right) \neq 0$.

Definition 6.7. An irreducible constituent $V(\nu)$ of $\mathfrak{l} \otimes \mathfrak{z}(\mathfrak{n})$ is called special 2 if $\nu \neq \gamma$ and there exists $\epsilon \in \Delta(\mathfrak{g}(1))$ so that $\nu=\mu+\epsilon$, where $\mu$ and $\gamma$ are the highest weights for $\mathfrak{g}(1)$ and $\mathfrak{z}(\mathfrak{n})$, respectively.

Proposition 6.8. Let $V(\nu)$ be an irreducible constituent of $\mathfrak{l} \otimes \mathfrak{z}(\mathfrak{n})$. Then $\left.\tilde{\tau}_{2}\right|_{V(\nu) *}$ is not identically zero only if $V(\nu)$ is a special constituent of $\mathfrak{l} \otimes \mathfrak{z}(\mathfrak{n})$.

Proof. At the beginning of this section we observed that if $\left.\tilde{\tau}_{2}\right|_{V(\nu)^{*}} \neq 0$, then $\nu$ must be of the form $\nu=\mu+\epsilon$ for some $\epsilon \in \Delta(\mathfrak{g}(1))$. Then $V(\nu)$ is either a special constituent or $V(\gamma)$ (by Lemma 4.14 $\gamma$ satisfies the form). However, by Proposition 6.5, it follows that $\left.\tilde{\tau}_{2}\right|_{V(\gamma)^{*}}$ is identically zero. Therefore, $V(\nu)$ must be a special constituent.

\footnotetext{
${ }^{2}$ Also see some comments in the introductions of [22] and 23] on the definition of special constituents.
} 
Now we determine all the special constituents of $\mathfrak{l} \otimes \mathfrak{z}(\mathfrak{n})$. Since $\mathfrak{l} \otimes \mathfrak{z}(\mathfrak{n})=$ $\left(\mathbb{C} H_{\mathfrak{q}} \otimes \mathfrak{z}(\mathfrak{n})\right) \oplus([\mathfrak{l}, \mathfrak{l}] \otimes \mathfrak{z}(\mathfrak{n}))$ and $\mathbb{C} H_{\mathfrak{q}} \otimes \mathfrak{z}(\mathfrak{n})=V(\gamma)$, it suffices to consider $[\mathfrak{l}, \mathfrak{l}] \otimes$ $\mathfrak{z}(\mathfrak{n})=\left(\mathfrak{l}_{\gamma} \otimes \mathfrak{z}(\mathfrak{n})\right) \oplus\left(\mathfrak{l}_{n \gamma} \otimes \mathfrak{z}(\mathfrak{n})\right)$. We start by observing that, by Proposition 6.2, $\mathfrak{l}_{n \gamma} \otimes \mathfrak{z}(\mathfrak{n})=V\left(\xi_{n \gamma}+\gamma\right)$.

Proposition 6.9. Suppose that $\mathfrak{l}_{n \gamma} \neq 0$. Then the irreducible constituent $V\left(\xi_{n \gamma}+\gamma\right)$ is special.

Proof. We need to show that $\xi_{n \gamma}+\gamma=\mu+\beta$ for some $\beta \in \Delta(\mathfrak{g}(1))$. This is precisely statement (1) of Lemma 4.16.

We next consider the constituent $V\left(\xi_{\gamma}+\gamma\right)$ of $\mathfrak{l}_{\gamma} \otimes \mathfrak{z}(\mathfrak{n})=V\left(\xi_{\gamma}\right) \otimes V(\gamma)$.

Lemma 6.10. The irreducible constituent $V\left(\xi_{\gamma}+\gamma\right)$ of $\mathfrak{l}_{\gamma} \otimes \mathfrak{z}(\mathfrak{n})$ is not special.

Proof. Lemma 4.16 and Remark 4.18 show that $\xi_{\gamma}+\gamma-\mu \notin \Delta(\mathfrak{g}(1))$, which implies that $\xi_{\gamma}+\gamma \neq \mu+\beta$ for all $\beta \in \Delta(\mathfrak{g}(1))$.

We determine all the special constituents of $\mathfrak{l}_{\gamma} \otimes \mathfrak{z}(\mathfrak{n})$ in two steps. First we assume that $\mathfrak{g}$ is a classical algebra, and then consider the case that $\mathfrak{g}$ is an exceptional algebra.

For classical cases the parabolic subalgebras $\mathfrak{q}$ under consideration are of type $B_{n}(i)(3 \leq i \leq n), C_{n}(i)(2 \leq i \leq n-1)$, or $D_{n}(i)(3 \leq i \leq n-3)$. It will be convenient to write $\beta \in \Delta(\mathfrak{g}(1))$ in terms of the fundamental weights of $\mathfrak{l}_{\gamma}$ and $\mathfrak{l}_{n \gamma}$. It is clear from the deleted Dynkin diagrams that, for each of the cases, $\Pi\left(\mathfrak{l}_{\gamma}\right)$ and $\Pi\left(\mathfrak{l}_{n \gamma}\right)$ are given by

$$
\Pi\left(\mathfrak{l}_{\gamma}\right)=\left\{\alpha_{r} \mid 1 \leq r \leq i-1\right\} \text { and } \Pi\left(\mathfrak{l}_{n \gamma}\right)=\left\{\alpha_{i+s} \mid 1 \leq s \leq n-i\right\},
$$

where $\alpha_{j}$ are the simple roots with the standard numbering. By using the standard realizations of roots, we have $\alpha_{r}=\varepsilon_{r}-\varepsilon_{r+1}$ for $1 \leq r \leq i-1, \alpha_{i+s}=\varepsilon_{i+s}-\varepsilon_{i+s+1}$ for $1 \leq s \leq n-i-1$, and

$$
\alpha_{n}= \begin{cases}\varepsilon_{n} & \text { if } \mathfrak{g} \text { is of type } B_{n}, \\ 2 \varepsilon_{n} & \text { if } \mathfrak{g} \text { is of type } C_{n} \\ \varepsilon_{n-1}+\varepsilon_{n} & \text { if } \mathfrak{g} \text { is of type } D_{n} .\end{cases}
$$

The data in Appendix $\mathrm{A}$ shows that if $\mathfrak{q}$ is of type $B_{n}(i)$, then

$$
\Delta(\mathfrak{g}(1))=\left\{\varepsilon_{j} \pm \varepsilon_{k} \mid 1 \leq j \leq i \text { and } i+1 \leq k \leq n\right\} \cup\left\{\varepsilon_{j} \mid 1 \leq j \leq i\right\},
$$

and if $\mathfrak{q}$ is of type $C_{n}(i)$ or $D_{n}(i)$, then

$$
\Delta(\mathfrak{g}(1))=\left\{\varepsilon_{j} \pm \varepsilon_{k} \mid 1 \leq j \leq i \text { and } i+1 \leq k \leq n\right\} .
$$

Since we have two simple algebras $\mathfrak{l}_{\gamma}$ and $\mathfrak{l}_{n \gamma}$, we use the notation $\varpi_{r}$ for the fundamental weights of $\alpha_{r} \in \Pi\left(\mathfrak{l}_{\gamma}\right)$ and $\tilde{\varpi}_{s}$ for those of $\alpha_{i+s} \in \Pi\left(\mathfrak{l}_{n \gamma}\right)$. Direct computation then shows that each $\beta \in \Delta(\mathfrak{g}(1))$ is exactly one of the following forms:

$$
\beta=\left\{\begin{array}{l}
\varpi_{1}+\sum_{s=1}^{n-i} \tilde{m}_{s} \tilde{\varpi}_{s}, \\
\left(-\varpi_{r}+\varpi_{r+1}\right)+\sum_{s=1}^{n-i} \tilde{m}_{s} \tilde{\varpi}_{s} \text { with } 1 \leq r \leq i-2, \text { or } \\
-\varpi_{i-1}+\sum_{s=1}^{n-i} \tilde{m}_{s} \tilde{\varpi}_{s}
\end{array}\right.
$$

for some $\tilde{m}_{s} \in \mathbb{Z}$.

Proposition 6.12. Let $V(\nu)$ be an irreducible constituent of $\mathfrak{l}_{\gamma} \otimes \mathfrak{z}(\mathfrak{n})$. 
(1) If $\mathfrak{q}$ is of type $B_{n}(i)(3 \leq i \leq n)$ or $D_{n}(i)(3 \leq i \leq n-3)$, then $V(\nu)$ is a special constituent if and only if $\nu=2 \varepsilon_{1}$.

(2) If $\mathfrak{q}$ is of type $C_{n}(i)(2 \leq i \leq n-1)$, then $V(\nu)$ is a special constituent if and only if $\nu=\varepsilon_{1}+\varepsilon_{2}$.

Proof. Suppose that $\mathfrak{q}$ is of type $B_{n}(i), C_{n}(i)$, or $D_{n}(i)$. By Definition 6.7, we need to find all $\nu$ of the form $\nu=\mu+\beta$ for some $\beta \in \Delta(\mathfrak{g}(1))$. Here $\mu$, the highest weight for $\mathfrak{g}(1)$, is

$$
\mu= \begin{cases}\varepsilon_{1}+\varepsilon_{i+1} & \text { if } \mathfrak{q} \text { is of type } B_{n}(i) \text { with } i \neq n, C_{n}(i), \text { or } D_{n}(i), \\ \varepsilon_{1} & \text { if } \mathfrak{q} \text { is of type } B_{n}(n) .\end{cases}
$$

We write $\mu$ in terms of the fundamental weights of $\mathfrak{l}_{\gamma}$ and $\mathfrak{l}_{n \gamma}$, that is,

$$
\mu= \begin{cases}\varpi_{1}+\tilde{\varpi}_{1} & \text { if } \mathfrak{q} \text { is of type } B_{n}(i) \text { with } i \neq n, C_{n}(i), \text { or } D_{n}(i), \\ \varpi_{1} & \text { if } \mathfrak{q} \text { is of type } B_{n}(n),\end{cases}
$$

where $\varpi_{1}$ and $\tilde{\varpi}_{1}$ are the fundamental weights of $\alpha_{1}=\varepsilon_{1}-\varepsilon_{2}$ and $\alpha_{i+1}=\varepsilon_{i+1}-$ $\varepsilon_{i+2}$, respectively. As $\mathfrak{l}_{n \gamma}$ acts trivially on both $\mathfrak{l}_{\gamma}$ and $\mathfrak{z}(\mathfrak{n})$, the highest weight $\nu$ for a constituent $V(\nu) \subset \mathfrak{l}_{\gamma} \otimes \mathfrak{z}(\mathfrak{n})$ is of the form

$$
\nu=\sum_{j=1}^{i-1} n_{j} \varpi_{j} \quad \text { for } n_{j} \in \mathbb{Z}_{\geq 0} .
$$

If there exists $\beta \in \Delta(\mathfrak{g}(1))$ so that $\nu=\mu+\beta$, then (6.13) and (6.14) imply that $\beta=\nu-\mu$ is of the form

$(6.15)$

$$
\begin{cases}\left(n_{1}-1\right) \varpi_{1}+\sum_{j=2}^{i-1} n_{j} \varpi_{j}-\tilde{\varpi}_{1} & \text { if } \mathfrak{q} \text { is of type } B_{n}(i) i \neq n, C_{n}(i), \text { or } D_{n}(i), \\ \left(n_{1}-1\right) \varpi_{1}+\sum_{j=2}^{i-1} n_{j} \varpi_{j} & \text { if } \mathfrak{q} \text { is of type } B_{n}(n)\end{cases}
$$

for $n_{j} \in \mathbb{Z}_{\geq 0}$. On the other hand, we observed that the root $\beta$ must be one of the forms in (6.11). Then observation shows that if $\beta$ satisfies both (6.11) and (6.15), then $\beta$ must be

$$
\begin{cases}\varpi_{1}-\tilde{\varpi}_{1} \text { or }\left(-\varpi_{1}+\varpi_{2}\right)-\tilde{\varpi}_{1} & \text { if } \mathfrak{q} \text { is of type } B_{n}(i) i \neq n, C_{n}(i), \text { or } D_{n}(i), \\ \varpi_{1} \text { or }\left(-\varpi_{1}+\varpi_{2}\right) & \text { if } \mathfrak{q} \text { is of type } B_{n}(n) .\end{cases}
$$

Therefore $\nu=\mu+\beta$ is $\nu=2 \varpi_{1}$ or $\varpi_{2}$, which shows that $\nu=2 \varepsilon_{1}$ or $\varepsilon_{1}+\varepsilon_{2}$. As $\xi_{\gamma}=\varepsilon_{1}-\varepsilon_{i}$ for $\mathfrak{q}$ of type $B_{n}(i), C_{n}(i)$, or $D_{n}(i)$, Theorem 6.3 shows that both $V\left(2 \varepsilon_{1}\right)$ and $V\left(\varepsilon_{1}+\varepsilon_{2}\right)$ occur in $\mathfrak{l}_{\gamma} \otimes \mathfrak{z}(\mathfrak{n})$. Now the assertions follow from the fact that the highest root $\gamma$ of $\mathfrak{g}$ is $\gamma=\varepsilon_{1}+\varepsilon_{2}$ if $\mathfrak{g}$ is of type $B_{n}$ or $D_{n}$, and $\gamma=2 \varepsilon_{1}$ if $\mathfrak{g}$ is of type $C_{n}$.

If $\mathfrak{g}$ is an exceptional algebra, then the parabolic subalgebras $\mathfrak{q}$ under consideration are

$$
E_{6}(3), E_{6}(5), E_{7}(2), E_{7}(6), E_{8}(1) \text {, and } F_{4}(4) \text {. }
$$

Lemma 6.17. If $\mathfrak{q}$ is of exceptional type as in (6.16), then $V\left(\xi_{\gamma}+\gamma_{0}\right)$ in Theorem 6.3 is a special constituent.

Proof. This is done by a direct computation. The roots $\epsilon_{\gamma}$ in $\Delta(\mathfrak{g}(1))$ so that $\xi_{\gamma}+\gamma_{0}=\mu+\epsilon_{\gamma}$ are given in Table 5 below.

Proposition 6.18. There exists a unique special constituent in $\mathfrak{l}_{\gamma} \otimes \mathfrak{z}(\mathfrak{n})$. 
Proof. If $\mathfrak{q}$ is of classical type, then this proposition follows from Proposition 6.12, For $\mathfrak{q}$ of exceptional type, by Theorem 6.3. the tensor product $\mathfrak{l}_{\gamma} \otimes \mathfrak{z}(\mathfrak{n})$ decomposes into

$$
\mathfrak{l}_{\gamma} \otimes \mathfrak{z}(\mathfrak{n})=V\left(\xi_{\gamma}+\gamma\right) \oplus V(\gamma) \oplus V\left(\xi_{\gamma}+\gamma_{0}\right)
$$

with $\gamma_{0} \in \Delta(\mathfrak{n})$ as in Theorem 6.3. Then Lemma 6.10 and Lemma 6.17 show that $V\left(\xi_{\gamma}+\gamma_{0}\right)$ is the unique special constituent.

Since the weight $\epsilon \in \Delta(\mathfrak{g}(1))$ so that $\mu+\epsilon$ is the highest weight of a special constituent will play a role later, we introduce the notation related to $\epsilon$.

Definition 6.19. We denote by $\epsilon_{\gamma}$ the root contributing to $\mathfrak{g}(1)$ so that $V\left(\mu+\epsilon_{\gamma}\right)$ is the special constituent of $\mathfrak{l}_{\gamma} \otimes \mathfrak{z}(\mathfrak{n})$. Similarly, we denote by $\epsilon_{n \gamma}$ the root for $\mathfrak{g}(1)$ so that $V\left(\mu+\epsilon_{n \gamma}\right)=\mathfrak{l}_{n \gamma} \otimes \mathfrak{z}(\mathfrak{n})$.

We summarize data on the special constituents in Table 2, Table 3 Table 4 , and Table 5. A dash indicates that no special constituent of the type exists for the case.

TABLE 2. Highest weights for special constituents (Classical cases)

\begin{tabular}{ccc}
\hline Type & $V\left(\mu+\epsilon_{\gamma}\right)$ & $V\left(\mu+\epsilon_{n \gamma}\right)$ \\
\hline$B_{n}(i), 3 \leq i \leq n-2$ & $2 \varepsilon_{1}$ & $\varepsilon_{1}+\varepsilon_{2}+\varepsilon_{i+1}+\varepsilon_{i+2}$ \\
$B_{n}(n-1)$ & $2 \varepsilon_{1}$ & $\varepsilon_{1}+\varepsilon_{2}+\varepsilon_{n}$ \\
$B_{n}(n)$ & $2 \varepsilon_{1}$ & - \\
$C_{n}(i), 2 \leq i \leq n-1$ & $\varepsilon_{1}+\varepsilon_{2}$ & $2 \varepsilon_{1}+2 \varepsilon_{i+1}$ \\
$D_{n}(i), 3 \leq i \leq n-3$ & $2 \varepsilon_{1}$ & $\varepsilon_{1}+\varepsilon_{2}+\varepsilon_{i+1}+\varepsilon_{i+2}$ \\
\hline
\end{tabular}

TABLE 3. Highest weights for special constituents (Exceptional cases)

\begin{tabular}{cc}
\hline Type & $V\left(\mu+\epsilon_{\gamma}\right)$ \\
\hline$E_{6}(3)$ & $\alpha_{1}+2 \alpha_{2}+2 \alpha_{3}+4 \alpha_{4}+3 \alpha_{5}+2 \alpha_{6}$ \\
$E_{6}(5)$ & $2 \alpha_{1}+2 \alpha_{2}+3 \alpha_{3}+4 \alpha_{4}+2 \alpha_{5}+\alpha_{6}$ \\
$E_{7}(2)$ & $2 \alpha_{1}+2 \alpha_{2}+4 \alpha_{3}+5 \alpha_{4}+4 \alpha_{5}+3 \alpha_{6}+2 \alpha_{7}$ \\
$E_{7}(6)$ & $2 \alpha_{1}+3 \alpha_{2}+4 \alpha_{3}+6 \alpha_{4}+4 \alpha_{5}+2 \alpha_{6}+\alpha_{7}$ \\
$E_{8}(1)$ & $2 \alpha_{1}+4 \alpha_{2}+5 \alpha_{3}+8 \alpha_{4}+7 \alpha_{5}+6 \alpha_{6}+4 \alpha_{7}+2 \alpha_{8}$ \\
$F_{4}(4)$ & $2 \alpha_{1}+4 \alpha_{2}+6 \alpha_{3}+2 \alpha_{4}$ \\
\hline Type & $V\left(\mu+\epsilon_{n \gamma}\right)$ \\
\hline$E_{6}(3)$ & $2 \alpha_{1}+2 \alpha_{2}+2 \alpha_{3}+3 \alpha_{4}+2 \alpha_{5}+\alpha_{6}$ \\
$E_{6}(5)$ & $\alpha_{1}+2 \alpha_{2}+2 \alpha_{3}+3 \alpha_{4}+2 \alpha_{5}+2 \alpha_{6}$ \\
$E_{7}(2)$ & - \\
$E_{7}(6)$ & $2 \alpha_{1}+2 \alpha_{2}+3 \alpha_{3}+4 \alpha_{4}+3 \alpha_{5}+2 \alpha_{6}+2 \alpha_{7}$ \\
$E_{8}(1)$ & - \\
$F_{4}(4)$ & - \\
\hline
\end{tabular}

By Proposition 6.8, only special constituents could contribute to the construction of the $\Omega_{2}$ systems. Next we want to show that $\left.\tilde{\tau}_{2}\right|_{V^{*}} \neq 0$ when $V$ is a special constituent. An observation on the highest weights for the special constituents will simplify the argument. We classify them by their highest weights and call them type $1 \mathrm{a}$, type $1 \mathrm{~b}$, type 2 , and type 3 . 
TABLE 4 . Roots $\mu, \epsilon_{\gamma}$, and $\epsilon_{n \gamma}$ (Classical cases)

\begin{tabular}{cccc}
\hline Type & $\mu$ & $\epsilon_{\gamma}$ & $\epsilon_{n \gamma}$ \\
\hline$B_{n}(i), 3 \leq i \leq n-2$ & $\varepsilon_{1}+\varepsilon_{i+1}$ & $\varepsilon_{1}-\varepsilon_{i+1}$ & $\varepsilon_{2}+\varepsilon_{i+2}$ \\
$B_{n}(n-1)$ & $\varepsilon_{1}+\varepsilon_{n}$ & $\varepsilon_{1}-\varepsilon_{n}$ & $\varepsilon_{2}$ \\
$B_{n}(n)$ & $\varepsilon_{1}$ & $\varepsilon_{1}$ & - \\
$C_{n}(i), 2 \leq i \leq n-1$ & $\varepsilon_{1}+\varepsilon_{i+1}$ & $\varepsilon_{2}-\varepsilon_{i+1}$ & $\varepsilon_{1}+\varepsilon_{i+1}$ \\
$D_{n}(i), 3 \leq i \leq n-3$ & $\varepsilon_{1}+\varepsilon_{i+1}$ & $\varepsilon_{1}-\varepsilon_{i+1}$ & $\varepsilon_{2}+\varepsilon_{i+2}$ \\
\hline
\end{tabular}

TABLE 5. Roots $\mu, \epsilon_{\gamma}$, and $\epsilon_{n \gamma}$ (Exceptional cases)

\begin{tabular}{cc}
\hline Type & $\mu$ \\
\hline$E_{6}(3)$ & $\alpha_{1}+\alpha_{2}+\alpha_{3}+2 \alpha_{4}+2 \alpha_{5}+\alpha_{6}$ \\
$E_{6}(5)$ & $\alpha_{1}+\alpha_{2}+2 \alpha_{3}+2 \alpha_{4}+\alpha_{5}+\alpha_{6}$ \\
$E_{7}(2)$ & $\alpha_{1}+\alpha_{2}+2 \alpha_{3}+3 \alpha_{4}+3 \alpha_{5}+2 \alpha_{6}+\alpha_{7}$ \\
$E_{7}(6)$ & $\alpha_{1}+2 \alpha_{2}+2 \alpha_{3}+3 \alpha_{4}+2 \alpha_{5}+\alpha_{6}+\alpha_{7}$ \\
$E_{8}(1)$ & $\alpha_{1}+3 \alpha_{2}+3 \alpha_{3}+5 \alpha_{4}+4 \alpha_{5}+3 \alpha_{6}+2 \alpha_{7}+\alpha_{8}$ \\
$F_{4}(4)$ & $\alpha_{1}+2 \alpha_{2}+3 \alpha_{3}+\alpha_{4}$ \\
\hline Type & $\epsilon_{\gamma}$ \\
\hline$E_{6}(3)$ & $\alpha_{2}+\alpha_{3}+2 \alpha_{4}+\alpha_{5}+\alpha_{6}$ \\
$E_{6}(5)$ & $\alpha_{1}+\alpha_{2}+\alpha_{3}+2 \alpha_{4}+\alpha_{5}$ \\
$E_{7}(2)$ & $\alpha_{1}+\alpha_{2}+2 \alpha_{3}+2 \alpha_{4}+\alpha_{5}+\alpha_{6}+\alpha_{7}$ \\
$E_{7}(6)$ & $\alpha_{1}+\alpha_{2}+2 \alpha_{3}+3 \alpha_{4}+2 \alpha_{5}+\alpha_{6}$ \\
$E_{8}(1)$ & $\alpha_{1}+\alpha_{2}+2 \alpha_{3}+3 \alpha_{4}+3 \alpha_{5}+3 \alpha_{6}+2 \alpha_{7}+\alpha_{8}$ \\
$F_{4}(4)$ & $\alpha_{1}+2 \alpha_{2}+3 \alpha_{3}+\alpha_{4}$ \\
\hline Type & $\epsilon_{n \gamma}$ \\
\hline$E_{6}(3)$ & $\alpha_{1}+\alpha_{2}+\alpha_{3}+\alpha_{4}$ \\
$E_{6}(5)$ & - \\
$E_{7}(2)$ & - \\
$E_{7}(6)$ & - \\
$E_{8}(1)$ & $\alpha_{2}+\alpha_{5}+\alpha_{6}$ \\
$F_{4}(4)$ & - \\
\hline
\end{tabular}

Definition 6.20. Let $\mu$ be the highest weight for $\mathfrak{g}(1)$, and let $\epsilon=\epsilon_{\gamma}$ or $\epsilon=\epsilon_{n \gamma}$. (See Definition 6.19]) We say that a special constituent $V(\mu+\epsilon)$ of $\mathfrak{l} \otimes \mathfrak{z}(\mathfrak{n})$ is of

(1) type $1 \mathrm{a}$ if $\mu+\epsilon$ is not a root with $\epsilon \neq \mu$ and both $\mu$ and $\epsilon$ are long roots,

(2) type $\mathbf{1 b}$ if $\mu+\epsilon$ is not a root with $\epsilon \neq \mu$ and either $\mu$ or $\epsilon$ is a short root,

(3) type 2 if $\mu+\epsilon=2 \mu$ is not a root, or

(4) type 3 if $\mu+\epsilon$ is a root.

Table 6 summarizes the types of special constituents for each parabolic subalgebra $\mathfrak{q}$. One may want to observe that almost all the special constituents are of type 1a. We regard any roots as long roots when $\mathfrak{g}$ is simply laced. A dash indicates that no special constituent of the type exists in the case.

Remark 6.21. It is observed from Table 4 and Table 5 that we have $\mu \pm \epsilon \notin \Delta$, unless $V(\mu+\epsilon)$ is of type 3. In particular, if $V(\mu+\epsilon)$ is of type 1a, then $\langle\mu, \epsilon\rangle=0$.

Remark 6.22. Table 6 shows that when $V(\mu+\epsilon)$ is a special constituent of type $1 \mathrm{a}, \mathfrak{q}$ is of type $B_{n}(i)(3 \leq i \leq n-1), D_{n}(i), E_{6}(3), E_{6}(5), E_{7}(2), E_{7}(6)$, or $E_{8}(1)$. 
TABLE 6. Types of special constituents

\begin{tabular}{ccc}
\hline Type & $V\left(\mu+\epsilon_{\gamma}\right)$ & $V\left(\mu+\epsilon_{n \gamma}\right)$ \\
\hline$B_{n}(i), 3 \leq i \leq n-2$ & type 1a & type 1a \\
$B_{n}(n-1)$ & type 1a & type 1b \\
$B_{n}(n)$ & type 2 & - \\
$C_{n}(i), 2 \leq i \leq n-1$ & type 3 & type 2 \\
$D_{n}(i), 3 \leq i \leq n-3$ & type 1a & type 1a \\
$E_{6}(3)$ & type 1a & type 1a \\
$E_{6}(5)$ & type 1a & type 1a \\
$E_{7}(2)$ & type 1a & - \\
$E_{7}(6)$ & type 1a & type 1a \\
$E_{8}(1)$ & type 1a & - \\
$F_{4}(4)$ & type 2 & - \\
\hline
\end{tabular}

The data in Appendix $\mathrm{A}$ shows that when $\mathfrak{q}$ is of type $B_{n}(i)$ for $3 \leq i \leq n-1$, the simple root $\alpha_{\mathfrak{q}}=\varepsilon_{i}-\varepsilon_{i+1}$ that parametrizes $\mathfrak{q}$ is a long root and that the set $\Delta(\mathfrak{z}(\mathfrak{n}))$ contains solely long roots. Since we regard any roots as long roots for $\mathfrak{g}$ simply laced, it follows that when $V(\mu+\epsilon)$ is of type 1a, the simple root $\alpha_{\mathfrak{q}}$ and any root $\gamma_{j} \in \Delta(\mathfrak{z}(\mathfrak{n}))$ are all long roots.

6.3. Computations for structure constants. For the rest of this section, we collect technical results on the special constituents and structure constants, so that certain arguments will go smoothly when we find the special values for the $\Omega_{2}$ systems. The root vectors $X_{\alpha}$ and the structure constants $N_{\alpha, \beta}$ are normalized as in Section 5.

Lemma 6.23. Let $V(\mu+\epsilon)$ be a special constituent $\mathfrak{l} \otimes \mathfrak{z}(\mathfrak{n})$ of type 1 a, and $\alpha \in$ $\Delta^{+}(\mathfrak{l})$. If $\epsilon+\alpha \in \Delta$, then $\mu-\alpha \in \Delta$.

Proof. We show that $\langle\mu, \alpha\rangle>0$. Since $\mu+\epsilon$ is the highest weight of an irreducible $\mathfrak{l}$-module, it is $\Delta(\mathfrak{l})$-dominant. Thus,

$$
\langle\mu+\epsilon, \alpha\rangle=\langle\mu, \alpha\rangle+\langle\epsilon, \alpha\rangle \geq 0 .
$$

Observe that, as $\mu+\epsilon$ is of type $1 \mathrm{a}, \epsilon$ is a long root of $\mathfrak{g}$. Since $\alpha+\epsilon$ is assumed to be a root, Lemma 4.15implies that $\left\langle\alpha, \epsilon^{\vee}\right\rangle=-1$; in particular, $\langle\epsilon, \alpha\rangle<0$. Now, by (6.24), we have

$$
\langle\mu, \alpha\rangle \geq-\langle\epsilon, \alpha\rangle>0 \text {. }
$$

Lemma 6.25. Let $V(\mu+\epsilon)$ be a special constituent of $\mathfrak{l} \otimes \mathfrak{z}(\mathfrak{n})$ of type 1a. If $\alpha \in \Delta^{+}(\mathfrak{l})$ with $\alpha+\epsilon \in \Delta$, then, for all $\gamma_{j} \in \Delta(\mathfrak{z}(\mathfrak{n}))$, we have

$$
\operatorname{ad}\left(X_{\mu}\right) \operatorname{ad}\left(X_{\alpha+\epsilon}\right) X_{-\gamma_{j}}=0 .
$$

Proof. If $(\alpha+\epsilon)-\gamma_{j} \notin \Delta$, then there is nothing to prove. So we assume that $(\alpha+\epsilon)-\gamma_{j} \in \Delta$ and $\mu+(\alpha+\epsilon)-\gamma_{j} \in \Delta$. Since $\mu+\epsilon$ is assumed to be of type 1a, the root $\mu$ is long. Lemma 4.15 then implies that

$$
\left\langle(\alpha+\epsilon)-\gamma_{j}, \mu^{\vee}\right\rangle=-1 .
$$

By Remark 6.21, we have $\left\langle\epsilon, \mu^{\vee}\right\rangle=0$. Thus (6.26) becomes

$$
\left\langle\alpha, \mu^{\vee}\right\rangle-\left\langle\gamma_{j}, \mu^{\vee}\right\rangle=-1 \text {. }
$$


Since $\mu$ is the highest weight for $\mathfrak{g}(1), \gamma_{j} \in \Delta(\mathfrak{z}(\mathfrak{n}))$, and $\alpha \in \Delta^{+}(\mathfrak{l})$, neither $\mu+\alpha$ nor $\gamma_{j}+\mu$ is a root. Then, as $\mu$ is a long root, (6.27) holds if and only if $\left\langle\alpha, \mu^{\vee}\right\rangle=0$ and $\left\langle\gamma_{j}, \mu^{\vee}\right\rangle=1$. On the other hand, since $\alpha+\epsilon$ is a root by hypothesis and by Lemma 6.23 $\mu-\alpha$ is a root. In particular, by Lemma 4.15, $\left\langle\alpha, \mu^{\vee}\right\rangle=1$. Now we have $\left\langle\alpha, \mu^{\vee}\right\rangle=1$ and $\left\langle\alpha, \mu^{\vee}\right\rangle=0$, which is a contradiction.

For any $\operatorname{ad}(\mathfrak{h})$-invariant subspace $W \subset \mathfrak{g}$ and any weight $\nu \in \mathfrak{h}^{*}$, we write

$$
\Delta_{\nu}(W)=\{\alpha \in \Delta(W) \mid \nu-\alpha \in \Delta\} .
$$

In Section 7 we will construct the $\left.\Omega_{2}\right|_{V(\mu+\epsilon) *}$ systems and find their special values when $V(\mu+\epsilon)$ is of either type 1a or type 2. When we do so, the roots $\beta \epsilon$ $\Delta_{\mu+\epsilon}(\mathfrak{g}(1))$ and $\gamma_{j} \in \Delta_{\mu+\epsilon}(\mathfrak{z}(\mathfrak{n}))$ will play a role. Therefore, for the rest of this section, we shall show several technical results about those roots so that certain arguments will become simple.

First of all, we need to check that $\Delta_{\mu+\epsilon}(\mathfrak{g}(1))$ and $\Delta_{\mu+\epsilon}(\mathfrak{z}(\mathfrak{n}))$ are not empty. It is clear that $\Delta_{\mu+\epsilon}(\mathfrak{g}(1)) \neq \emptyset$, since $\mu, \epsilon \in \Delta_{\mu+\epsilon}(\mathfrak{g}(1))$. Moreover, Lemma 6.28 below shows that when $V(\mu+\epsilon)$ is of type 2, we have $\Delta_{\mu+\epsilon}(\mathfrak{g}(1))=\{\mu\}$.

Lemma 6.28. If $V(\mu+\epsilon)$ is a special constituent of $\mathfrak{l} \otimes \mathfrak{z}(\mathfrak{n})$ of type 2, then $\Delta_{\mu+\epsilon}(\mathfrak{g}(1))=\{\mu\}$.

Proof. First we claim that $\mu$ has the maximum height among the roots $\beta \in \Delta(\mathfrak{g}(1))$. As $\mathfrak{g}(1)$ is the irreducible $L$-module with highest weight $\mu$, any $\operatorname{root} \beta \in \Delta(\mathfrak{g}(1))$ is of the form $\beta=\mu-\sum_{\alpha \in \Pi(\mathfrak{l})} n_{\alpha} \alpha$ with $n_{\alpha} \in \mathbb{Z}_{\geq 0}$. Then if $\operatorname{ht}(\mu)$ and $\operatorname{ht}(\beta)$ denote the heights of $\mu$ and $\beta$, respectively, then

$$
\operatorname{ht}(\mu)=\operatorname{ht}(\beta)+\sum_{\alpha \in \Pi(\mathfrak{l})} n_{\alpha} \geq \operatorname{ht}(\beta) .
$$

Now as $V(\mu+\epsilon)$ is of type 2 , by definition, we have $\mu+\epsilon=2 \mu$. If $\beta \in \Delta_{2 \mu}(\mathfrak{g}(1))$, then $2 \mu-\beta \in \Delta(\mathfrak{g}(1))$. In particular, the height ht $(2 \mu-\beta)$ satisfies $\operatorname{ht}(\mu) \geq$ ht $(2 \mu-\beta)$. If $\beta=\mu-\sum_{\alpha \in \Pi(\mathfrak{l})} n_{\alpha} \alpha$ with $n_{\alpha} \in \mathbb{Z}_{\geq 0}$, then

$$
\operatorname{ht}(\mu) \geq \operatorname{ht}(2 \mu-\beta)=2 \operatorname{ht}(\mu)-\operatorname{ht}(\beta)=\operatorname{ht}(\mu)+\sum_{\alpha \in \Pi(\mathfrak{l})} n_{\alpha} .
$$

This forces that $\sum_{\alpha \in \Pi(\mathfrak{l})} n_{\alpha}=0$. Therefore $\beta=\mu$.

Lemma 6.29. If $V(\mu+\epsilon)$ is a special constituent of $\mathfrak{l} \otimes \mathfrak{z}(\mathfrak{n})$, then $\Delta_{\mu+\epsilon}(\mathfrak{z}(\mathfrak{n})) \neq \emptyset$.

Proof. Observe that the highest weight $\mu+\epsilon$ of $V(\mu+\epsilon) \subset \mathfrak{l} \otimes \mathfrak{z}(\mathfrak{n})$ must be of the form

$$
\mu+\epsilon= \begin{cases}\xi_{\gamma}+\gamma^{\prime} & \text { if } V(\mu+\epsilon) \subset \mathfrak{l}_{\gamma} \otimes \mathfrak{z}(\mathfrak{n}), \\ \xi_{n \gamma}+\gamma^{\prime \prime} & \text { if } V(\mu+\epsilon)=\mathfrak{l}_{n \gamma} \otimes \mathfrak{z}(\mathfrak{n})\end{cases}
$$

for some $\gamma^{\prime}, \gamma^{\prime \prime} \in \Delta(\mathfrak{z}(\mathfrak{n}))$, where $\xi_{\gamma}$ and $\xi_{n \gamma}$ are the highest weights for $\mathfrak{l}_{\gamma}$ and $\mathfrak{l}_{n \gamma}$, respectively. Then we have $\gamma^{\prime}, \gamma^{\prime \prime} \in \Delta_{\mu+\epsilon}(\mathfrak{z}(\mathfrak{n}))$.

The following simple technical lemma will simplify an argument in later proofs.

Lemma 6.30. Let $\alpha, \beta, \delta \in \Delta$ with $\alpha, \beta \neq \delta$. If $\alpha+\beta \notin \Delta$ and $\alpha+\beta-\delta \in \Delta$, then the following hold:

(1) $\alpha-\delta, \beta-\delta \in \Delta$, and

(2) $N_{\beta, \alpha-\delta} N_{\alpha,-\delta}=N_{\alpha, \beta-\delta} N_{\beta,-\delta}$. 
Proof. These simply follow from the structure of the complex simple Lie algebras.

Lemma 6.31. Let $W$ be any ad(h)-invariant subspace of $\mathfrak{g}$ with the condition $\Delta_{\mu+\epsilon}(W) \backslash\{\mu, \epsilon\} \neq \emptyset$. If $V(\mu+\epsilon)$ is a special constituent of $\mathfrak{l} \otimes \mathfrak{z}(\mathfrak{n})$ of type 1a, type $1 b$, or type 2, then, for any $\delta \in \Delta_{\mu+\epsilon}(W) \backslash\{\mu, \epsilon\}$, we have $\delta-\mu, \delta-\epsilon \in \Delta$.

Proof. If $V(\mu+\epsilon)$ is of type 1a, type $1 \mathrm{~b}$, or type 2 , then, by definition, $\mu+\epsilon$ is not a root. Then this lemma simply follows from Lemma 6.30.

Remark 6.32. A direct observation shows that if $V(\mu+\epsilon)$ is a special constituent of type 1a, then $\Delta_{\mu+\epsilon}(\mathfrak{g}(1)) \backslash\{\mu, \epsilon\} \neq \emptyset$.

Lemma 6.33. If $V(\mu+\epsilon)$ is a special constituent of $\mathfrak{l} \otimes \mathfrak{z}(\mathfrak{n})$ of type 1 a, then, for any $\alpha \in \Delta_{\mu+\epsilon}(\mathfrak{g}(1))$ and any $\gamma_{j} \in \Delta_{\mu+\epsilon}(\mathfrak{z}(\mathfrak{n}))$, we have $\gamma_{j}-\alpha \in \Delta$.

Proof. By Lemma 6.30, we have $\gamma_{j}-\mu, \gamma_{j}-\epsilon \in \Delta$. So, let $\alpha \neq \mu, \epsilon$. We show that $\left\langle\gamma_{j}, \alpha\right\rangle>0$. Observe that since $\alpha \in \Delta(\mathfrak{g}(1))$ and $\gamma_{j} \in \Delta(\mathfrak{z}(\mathfrak{n}))$, we have $\gamma_{j}+\alpha \notin \Delta$. Thus $\left\langle\gamma_{j}, \alpha\right\rangle \geq 0$. Since $\alpha \in \Delta_{\mu+\epsilon}(\mathfrak{g}(1)) \backslash\{\mu, \epsilon\}$ and $\gamma_{j} \in \Delta_{\mu+\epsilon}(\mathfrak{z}(\mathfrak{n}))$, by Lemma 6.31. we have $\mu-\alpha, \epsilon-\gamma_{j} \in \Delta$. Then we first claim that if $\left\langle\gamma_{j}, \alpha\right\rangle=0$, then $(\mu-\alpha)+\left(\epsilon-\gamma_{j}\right) \in \Delta$. Since $V(\mu+\epsilon)$ is assumed to be of type 1a, both $\mu$ and $\epsilon$ are long roots. Thus, by Lemma 4.15, $\left\langle\gamma_{j}, \mu^{\vee}\right\rangle=\left\langle\alpha, \epsilon^{\vee}\right\rangle=1$; in particular, $\left\langle\gamma_{j}, \mu\right\rangle$, $\langle\alpha, \epsilon\rangle>0$. By Remark 6.21, we have $\langle\mu, \epsilon\rangle=0$. Then,

$$
\left\langle\mu-\alpha, \epsilon-\gamma_{j}\right\rangle=-\left\langle\mu, \gamma_{j}\right\rangle-\langle\alpha, \epsilon\rangle<0 .
$$

Therefore, as $\mu-\alpha, \epsilon-\gamma_{j} \in \Delta$, it follows that $(\mu-\alpha)+\left(\epsilon-\gamma_{j}\right) \in \Delta$. On the other hand, since $\langle\mu, \epsilon\rangle=0$ and $\left\langle\gamma_{j}, \alpha\right\rangle$ is assumed to be 0 , we have

$$
\begin{aligned}
& \left\|(\mu-\alpha)+\left(\epsilon-\gamma_{j}\right)\right\|^{2} \\
& =\|\mu\|^{2}+\|\alpha\|^{2}+\|\epsilon\|^{2}+\left\|\gamma_{j}\right\|^{2}-2\langle\alpha, \mu\rangle-2\langle\alpha, \epsilon\rangle-2\left\langle\gamma_{j}, \mu\right\rangle-2\left\langle\gamma_{j}, \epsilon\right\rangle .
\end{aligned}
$$

For $\nu=\alpha, \gamma_{j}$ and $\zeta=\mu, \epsilon$, by Lemma 4.15 we have $\left\langle\nu, \zeta^{\vee}\right\rangle=2\langle\nu, \zeta\rangle /\|\zeta\|^{2}=1$, as $\mu$ and $\epsilon$ are long roots. Therefore, $2\langle\nu, \zeta\rangle=\|\zeta\|^{2}$, and so

$$
\left\|(\mu-\alpha)+\left(\epsilon-\gamma_{j}\right)\right\|^{2}=\|\alpha\|^{2}+\left\|\gamma_{j}\right\|^{2}-\|\mu\|^{2}-\|\epsilon\|^{2} .
$$

Since $\mu$ and $\epsilon$ are assumed to be long roots, this shows that $\left\|(\mu-\alpha)+\left(\epsilon-\gamma_{j}\right)\right\|^{2} \leq 0$, which contradicts that $(\mu-\alpha)+\left(\epsilon-\gamma_{j}\right)$ is a root. Hence, $\left\langle\gamma_{j}, \alpha\right\rangle>0$.

Lemma 6.34. If $V(\mu+\epsilon)$ is a special constituent of $\mathfrak{l} \otimes \mathfrak{z}(\mathfrak{n})$ of type 1 a or type 2 , then, for any $\gamma_{j} \in \Delta_{\mu+\epsilon}(\mathfrak{z}(\mathfrak{n}))$,

$$
\Delta_{\mu+\epsilon}(\mathfrak{g}(1)) \subset \Delta_{\gamma_{j}}(\mathfrak{g}(1)) .
$$

In particular, $\Delta_{\gamma_{j}}(\mathfrak{g}(1)) \neq \emptyset$ for any $\gamma_{j} \in \Delta_{\mu+\epsilon}(\mathfrak{z}(\mathfrak{n}))$.

Proof. It is clear that the type 1a case follows from Lemma 6.33. The type 2 case follows from Lemma 6.28 and Lemma 6.31

If $V(\mu+\epsilon)$ is a special constituent of $\mathfrak{l} \otimes \mathfrak{z}(\mathfrak{n})$, then, for $\beta \in \Delta$, we write

$$
\theta(\beta)=(\mu+\epsilon)-\beta .
$$

Lemma 6.35. If $V(\mu+\epsilon)$ is a special constituent of $\mathfrak{l} \otimes \mathfrak{z}(\mathfrak{n})$ of type 1 a or type 2 , then, for any $\gamma_{j} \in \Delta_{\mu+\epsilon}(\mathfrak{z}(\mathfrak{n}))$,

$$
\Delta_{\theta\left(\gamma_{j}\right)}(\mathfrak{g}(1)) \neq \emptyset
$$


Proof. This simply follows from Lemma 6.30.

Lemma 6.36. If $V(\mu+\epsilon)$ is a special constituent of type 1a or type 2, then

$$
\sum_{\gamma_{j} \in \Delta_{\mu+\epsilon}(\mathfrak{z}(\mathfrak{n}))} N_{\mu, \epsilon-\gamma_{j}} N_{-\mu, \gamma_{j}-\epsilon} N_{\epsilon,-\gamma_{j}} N_{-\epsilon, \gamma_{j}}>0,
$$

where $N_{\alpha, \beta}$ are the structure constants for $\alpha, \beta \in \Delta$ defined in Section 5 .

Proof. It follows from the normalization (H7) in Section 5 that

$$
N_{\mu, \epsilon-\gamma_{j}} N_{-\mu, \gamma_{j}-\epsilon}=-\frac{q_{\mu, \epsilon-\gamma_{j}}\left(1+p_{\mu, \epsilon-\gamma_{j}}\right)}{2}\|\mu\|^{2}
$$

and

$$
N_{\epsilon,-\gamma_{j}} N_{-\epsilon, \gamma_{j}}=-\frac{q_{\epsilon,-\gamma_{j}}\left(1+p_{\epsilon,-\gamma_{j}}\right)}{2}\|\epsilon\|^{2} .
$$

In particular, by (5.1) in Section [5. $N_{\mu, \epsilon-\gamma_{j}} N_{-\mu, \gamma_{j}-\epsilon} \leq 0$ and $N_{\epsilon,-\gamma_{j}} N_{-\epsilon, \gamma_{j}} \leq 0$. By Lemma 6.29 and Lemma 6.31, $\Delta_{\mu+\epsilon}(\mathfrak{z}(\mathfrak{n})) \neq \emptyset$ and $\gamma_{j}-\epsilon \in \Delta$ for any $\gamma_{j} \in$ $\Delta_{\mu+\epsilon}(\mathfrak{z}(\mathfrak{n}))$. Therefore, for all $\gamma_{j} \in \Delta_{\mu+\epsilon}(\mathfrak{z}(\mathfrak{n}))$, we have

$$
N_{\mu, \epsilon-\gamma_{j}} N_{-\mu, \gamma_{j}-\epsilon} N_{\epsilon,-\gamma_{j}} N_{-\epsilon, \gamma_{j}}>0 .
$$

Lemma 6.37. If $V(\mu+\epsilon)$ is a special constituent of type 1a, then, for any $\alpha \in$ $\Delta_{\mu+\epsilon}(\mathfrak{g}(1)) \backslash\{\mu, \epsilon\}$ and any $\gamma_{j} \in \Delta_{\mu+\epsilon}(\mathfrak{z}(\mathfrak{n}))$, we have the following:

(1) $\left[X_{-\gamma_{j}}, X_{\alpha-\mu}\right]=\left[X_{\theta\left(\gamma_{j}\right)}, X_{\alpha-\mu}\right]=0$.

(2) $N_{\mu-\gamma_{j}, \alpha-\mu} N_{-\left(\mu-\gamma_{j}\right),-(\alpha-\mu)}=-\frac{\left\|\mu-\gamma_{j}\right\|^{2}}{2}$.

Proof. To prove (1), we show that $-\gamma_{j}+\alpha-\mu$ and $\theta\left(\gamma_{j}\right)+\alpha-\mu$ are neither zero nor roots. First of all, if $-\gamma_{j}+\alpha-\mu=0$, then $\gamma_{j}=\mu-\alpha \in \Delta(\mathfrak{l})$, which contradicts that $\gamma_{j} \in \Delta(\mathfrak{z}(\mathfrak{n}))$. Next, if $\theta\left(\gamma_{j}\right)+\alpha-\mu=0$, then since $\theta\left(\gamma_{j}\right)+\alpha-\mu=\epsilon+\alpha-\gamma_{j}$, we would have $\alpha+\epsilon=\gamma_{j} \in \Delta$. On the other hand, as $V(\mu+\epsilon)$ is assumed to be of type 1a, $\epsilon$ is a long root. As $\alpha \in \Delta_{\mu+\epsilon}(\mathfrak{g}(1)) \backslash\{\mu, \epsilon\}$, by Lemma 6.31 we have $\alpha-\epsilon \in \Delta$. Then, by Lemma 4.15, it follows that $\alpha+\epsilon \notin \Delta$, which is a contradiction. To show $\gamma_{j}+\alpha-\mu$ is not a root, observe that, by Lemma 4.15, we have

$$
\left\langle-\gamma_{j}+\alpha-\mu, \mu^{\vee}\right\rangle=-1+1-2=-2 .
$$

Thus, if $-\gamma_{j}+\alpha-\mu \in \Delta$, then $\left(-\gamma_{j}+\alpha-\mu\right)+2 \mu$ would be a root. However, since $\mu$ is a long root, it is impossible. The fact that $\theta\left(\gamma_{j}\right)+\alpha-\mu \notin \Delta$ can be shown in a similar manner.

By the normalization (H7) in Section [5, to show (2), it suffices to show that $p_{\mu-\gamma_{j}, \alpha-\mu}=0$ and $q_{\mu-\gamma_{j}, \alpha-\mu}=1$. Observe that, by Lemma6.33. $(\alpha-\mu)+\left(\mu-\gamma_{j}\right)=$ $\gamma_{j}-\alpha$ is a root. As $V(\mu+\epsilon)$ is assumed to be of type 1a, $\mu$ is a long root. By Remark 6.22, the root $\gamma_{j}$ is also a long root. Therefore $\mu-\gamma_{j}$ is a long root. Now the proposed equality follows from Lemma 4.15.

Lemma 6.38. If $V(\mu+\epsilon)$ is a special constituent of type $1 a$, then, for any $\alpha \in$ $\Delta_{\mu+\epsilon}(\mathfrak{g}(1)) \backslash\{\mu, \epsilon\}$ and any $\gamma_{j} \in \Delta_{\mu+\epsilon}(\mathfrak{z}(\mathfrak{n}))$, we have the following:

(1) $N_{\alpha,-\gamma_{j}} N_{\mu,-\alpha}=N_{\mu,-\gamma_{j}} N_{\alpha-\mu, \mu-\gamma_{j}}$, and

(2) $N_{-\theta\left(\gamma_{j}\right), \theta(\alpha)} N_{-\theta(\alpha), \theta(\mu)}=N_{-\theta\left(\gamma_{j}\right), \theta(\mu)} N_{-\left(\mu-\gamma_{j}\right),-(\alpha-\mu)}$. 
Proof. It follows from Lemma 6.31 that $\alpha-\mu \in \Delta$. Therefore, we have $X_{\alpha}=$ $\left(1 / N_{\alpha-\mu, \mu}\right)\left[X_{\alpha-\mu}, X_{\mu}\right]$. Now assertion (1) follows from the Jacobi identity and the normalization (H6) with Lemma 6.33 and Lemma 6.37 (1). Assertion (2) can be shown similarly.

Lemma 6.39. Let $\mathfrak{q}$ be a parabolic subalgebra of quasi-Heisenberg type, listed in (4.9) or (4.10), and $\alpha_{\mathfrak{q}}$ be the simple root that parametrizes the parabolic subalgebra q. If $V(\mu+\epsilon)$ is a special constituent of type $1 a$, then, for any $\alpha \in \Delta_{\mu+\epsilon}(\mathfrak{g}(1)) \backslash\{\mu, \epsilon\}$ and any $\gamma_{j} \in \Delta_{\mu+\epsilon}(\mathfrak{z}(\mathfrak{n}))$,

$$
N_{\alpha,-\gamma_{j}} N_{\mu,-\alpha} N_{-\theta\left(\gamma_{j}\right), \theta(\alpha)} N_{-\theta(\alpha), \theta(\mu)}=N_{\mu, \epsilon-\gamma_{j}} N_{\epsilon,-\gamma_{j}} \frac{\left\|\alpha_{\mathfrak{q}}\right\|^{2}}{2} .
$$

Proof. By Lemma 6.38, we have

$$
\begin{aligned}
N_{\alpha,-\gamma_{j}} & N_{\mu,-\alpha} N_{-\theta\left(\gamma_{j}\right), \theta(\alpha)} N_{-\theta(\alpha), \theta(\mu)} \\
& =N_{\mu,-\gamma_{j}} N_{\alpha-\mu, \mu-\gamma_{j}} N_{-\theta\left(\gamma_{j}\right), \theta(\mu)} N_{-\left(\mu-\gamma_{j}\right),-(\alpha-\mu)} \\
& =N_{\mu,-\gamma_{j}} N_{-\theta\left(\gamma_{j}\right), \theta(\mu)} N_{\alpha-\mu, \mu-\gamma_{j}} N_{-\left(\mu-\gamma_{j}\right),-(\alpha-\mu)} \\
& =N_{\mu,-\gamma_{j}} N_{-\theta\left(\gamma_{j}\right), \theta(\mu)} \frac{\left\|\mu-\gamma_{j}\right\|^{2}}{2} .
\end{aligned}
$$

Note that Lemma 6.37 (2) is applied from line three to line four. Since $-\theta\left(\gamma_{j}\right)+$ $\theta(\mu)+\left(\mu-\gamma_{j}\right)=0$ with $\theta(\mu)=(\mu+\epsilon)-\mu=\epsilon$, by the normalization (H6), we have $N_{-\theta\left(\gamma_{j}\right), \theta(\mu)}=N_{\epsilon, \mu-\gamma_{j}}$. By Lemma 6.30 with $\alpha=\mu, \beta=\epsilon$, and $\delta=\gamma_{j}$, it follows that $N_{\epsilon, \mu-\gamma_{j}} N_{\mu,-\gamma_{j}}=N_{\mu, \epsilon-\gamma_{j}} N_{\epsilon,-\gamma_{j}}$. Therefore,

$$
N_{\mu,-\gamma_{j}} N_{-\theta\left(\gamma_{j}\right), \theta(\mu)}=N_{\mu,-\gamma_{j}} N_{\epsilon, \mu-\gamma_{j}}=N_{\mu, \epsilon-\gamma_{j}} N_{\epsilon,-\gamma_{j}} .
$$

Remark6.22 shows that $\gamma_{j}$ and $\alpha_{\mathfrak{q}}$ are long roots when $V(\mu+\epsilon)$ is of type 1a. Since $\mu$ is assumed to be a long root, the root $\mu-\gamma_{j}$ is a long root. Thus $\left\|\mu-\gamma_{j}\right\|^{2}=\left\|\alpha_{\mathfrak{q}}\right\|^{2}$. Hence,

$$
\begin{aligned}
N_{\alpha,-\gamma_{j}} N_{\mu,-\alpha} N_{-\theta\left(\gamma_{j}\right), \theta(\alpha)} N_{-\theta(\alpha), \theta(\mu)} & =N_{\mu,-\gamma_{j}} N_{-\theta\left(\gamma_{j}\right), \theta(\mu)} \frac{\left\|\mu-\gamma_{j}\right\|^{2}}{2} \\
& =N_{\mu, \epsilon-\gamma_{j}} N_{\epsilon,-\gamma_{j}} \frac{\left\|\alpha_{\mathfrak{q}}\right\|^{2}}{2} .
\end{aligned}
$$

\section{The $\Omega_{2}$ SYSTEMS}

We continue with $\mathfrak{q}=\mathfrak{l} \oplus \mathfrak{g}(1) \oplus \mathfrak{z}(\mathfrak{n})$ a maximal parabolic subalgebra of quasiHeisenberg type, listed in (4.9) or (4.10). In this section, by using the preliminary results from Section [6, we shall determine the complex parameter $s_{2} \in \mathbb{C}$ for the line bundle $\mathcal{L}_{s}$ so that the $\Omega_{2}$ systems are conformally invariant on $\mathcal{L}_{s_{2}}$. This is done in Theorem 7.16

7.1. Covariant map $\tau_{2}$. As we have observed in Subsection 3.1, to construct the $\left.\Omega_{2}\right|_{V^{*}}$ system, we use the covariant map $\tau_{2}$ and the associated $L$-intertwining operator $\left.\tilde{\tau}_{2}\right|_{V^{*}}$, where $V^{*}$ is an irreducible constituent of $\mathfrak{l}^{*} \otimes \mathfrak{z}(\mathfrak{n})^{*}=\mathfrak{g}(0)^{*} \otimes \mathfrak{g}(2)^{*}$. We first show that the covariant map $\tau_{2}$ is not identically zero, and also that the $L$-intertwining operators $\left.\tilde{\tau}_{2}\right|_{V^{*}}$ are not identically zero for certain irreducible constituents $V$. We continue to use the normalizations from Section 5. 
We start by showing that $\tau_{2}$ is not identically zero. The covariant map $\tau_{2}$ is given by

$$
\begin{aligned}
\tau_{2}: \mathfrak{g}(1) & \rightarrow \mathfrak{l} \otimes \mathfrak{z}(\mathfrak{n}), \\
X & \mapsto \frac{1}{2}\left(\operatorname{ad}(X)^{2} \otimes \mathrm{Id}\right) \omega
\end{aligned}
$$

with $\omega=\sum_{\gamma_{j} \in \Delta(\mathfrak{z}(\mathfrak{n}))} X_{-\gamma_{j}} \otimes X_{\gamma_{j}}$. The following technical lemma will make a certain argument simpler in later proofs.

Lemma 7.1. If $V(\mu+\epsilon)$ is a special constituent of type 1 a or type 2 , then

$$
\tau_{2}\left(X_{\mu}+X_{\epsilon}\right)=a_{\mu, \epsilon} \operatorname{ad}\left(X_{\mu}\right) \operatorname{ad}\left(X_{\epsilon}\right) \omega,
$$

where $a_{\mu, \epsilon}=1+\delta_{\mu, \epsilon}$ with $\delta_{\mu, \epsilon}$ the Kronecker delta.

Proof. It is clear that (7.2) holds if $\mu+\epsilon$ is of type 2. Indeed, if $\epsilon=\mu$, then we have

$$
\tau_{2}\left(2 X_{\mu}\right)=4 \tau_{2}\left(X_{\mu}\right)=2 \operatorname{ad}\left(X_{\mu}\right)^{2} \omega .
$$

If $\mu+\epsilon$ is of type 1a, then, by definition, $\mu+\epsilon \notin \Delta$ and both $\mu$ and $\epsilon$ are long roots. Thus, in the case, $\operatorname{ad}\left(X_{\mu}\right) \operatorname{ad}\left(X_{\epsilon}\right)=\operatorname{ad}\left(X_{\epsilon}\right) \operatorname{ad}\left(X_{\mu}\right)$. Moreover, by Lemma 4.15, we have $\operatorname{ad}\left(X_{\mu}\right)^{2} X_{-\gamma_{j}}=\operatorname{ad}\left(X_{\epsilon}\right)^{2} X_{-\gamma_{j}}=0$ for any $\gamma_{j} \in \Delta(\mathfrak{z}(\mathfrak{n}))$. Hence,

$$
\tau_{2}\left(X_{\mu}+X_{\epsilon}\right)=(1 / 2)\left(2 \operatorname{ad}\left(X_{\mu}\right) \operatorname{ad}\left(X_{\epsilon}\right)\right) \omega=\operatorname{ad}\left(X_{\mu}\right) \operatorname{ad}\left(X_{\epsilon}\right) \omega .
$$

Proposition 7.3. Let $\mathfrak{q}$ be a maximal parabolic subalgebra of quasi-Heisenberg type listed in (4.9) or (4.10). Then the covariant map $\tau_{2}$ is not identically zero.

Proof. To prove that $\tau_{2}$ is not identically zero, it suffices to show that there exists a vector $X \in \mathfrak{g}(1)$ so that $\tau_{2}(X) \neq 0$. Observe that, for each $\mathfrak{q}$ under consideration, $\mathfrak{l} \otimes \mathfrak{z}(\mathfrak{n})$ has at least one special constituent $V(\mu+\epsilon)$ of type 1a or type 2 (see Table 6] in Subsection 6.2). Therefore, $\Delta(\mathfrak{g}(1))$ always contains a root $\epsilon$ so that $V(\mu+\epsilon)$ is such a special constituent. Then, to prove this proposition, we show that $\tau_{2}\left(X_{\mu}+X_{\epsilon}\right) \neq 0$, where $X_{\mu}$ and $X_{\epsilon}$ are root vectors for $\mu$ and $\epsilon$, respectively, with $\mu+\epsilon$ the highest weight for a special constituent of type 1a or type 2 .

Let $\mu+\epsilon$ be the highest weight of a special constituent of type 1a or type 2. By Lemma 7.1 we have

$$
\tau_{2}\left(X_{\mu}+X_{\epsilon}\right)=a_{\mu, \epsilon} \operatorname{ad}\left(X_{\mu}\right) \operatorname{ad}\left(X_{\epsilon}\right) \omega=a_{\mu, \epsilon} \sum_{\gamma_{j} \in \Delta(\mathfrak{z}(\mathfrak{n}))} \operatorname{ad}\left(X_{\mu}\right) \operatorname{ad}\left(X_{\epsilon}\right) X_{-\gamma_{j}} \otimes X_{\gamma_{j}}
$$

with $a_{\mu, \epsilon}=1+\delta_{\mu, \epsilon}$. If there were a root $\gamma_{j} \in \Delta(\mathfrak{z}(\mathfrak{n}))$ such that $\epsilon-\gamma_{j}=-\mu$, then $\mu+\epsilon=\gamma_{j} \in \Delta$, which contradicts the assumption that $\mu+\epsilon$ is of type 1a or type 2. By Lemma 6.31, if $\mu+\epsilon-\gamma_{j} \in \Delta$, then $\epsilon-\gamma_{j} \in \Delta$. Then, for all $\gamma_{j} \in \Delta(\mathfrak{z}(\mathfrak{n}))$,

$$
\operatorname{ad}\left(X_{\mu}\right) \operatorname{ad}\left(X_{\epsilon}\right) X_{-\gamma_{j}}= \begin{cases}N_{\mu, \epsilon-\gamma_{j}} N_{\epsilon,-\gamma_{j}} X_{\mu+\epsilon-\gamma_{j}} & \text { if } \mu+\epsilon-\gamma_{j} \in \Delta, \\ 0 & \text { otherwise. }\end{cases}
$$

Therefore, we have

$$
\begin{aligned}
\tau_{2}\left(X_{\mu}+X_{\epsilon}\right) & =a_{\mu, \epsilon} \sum_{\gamma_{j} \in \Delta(\mathfrak{z}(\mathfrak{n}))} \operatorname{ad}\left(X_{\mu}\right) \operatorname{ad}\left(X_{\epsilon}\right) X_{-\gamma_{j}} \otimes X_{\gamma_{j}} \\
& =a_{\mu, \epsilon} \sum_{\gamma_{j} \in \Delta_{\mu+\epsilon}(\mathfrak{z}(\mathfrak{n}))} N_{\mu, \epsilon-\gamma_{j}} N_{\epsilon,-\gamma_{j}} X_{\mu+\epsilon-\gamma_{j}} \otimes X_{\gamma_{j}} .
\end{aligned}
$$


Since $\left\{X_{\mu+\epsilon-\gamma_{j}} \otimes X_{\gamma_{j}} \mid \gamma_{j} \in \Delta_{\mu+\epsilon}(\mathfrak{z}(\mathfrak{n}))\right\}$ is a linearly independent set, this shows that $\tau_{2}\left(X_{\mu}+X_{\epsilon}\right) \neq 0$.

Next we identify the irreducible constituent $V(\nu)^{*}$ so that $\left.\tilde{\tau}_{2}\right|_{V(\nu)^{*}}$ is not identically zero. In Subsection 3.1, we observed that, given an irreducible constituent $V(\nu)^{*}$, the $L$-intertwining operator $\left.\tilde{\tau}_{2}\right|_{V(\nu)^{*}} \in \operatorname{Hom}_{L}\left(V(\nu)^{*}, \mathcal{P}^{2}(\mathfrak{g}(1))\right)$ is given by

$$
\left.\tilde{\tau}_{2}\right|_{V(\nu)^{*}}\left(Y^{*}\right)(X)=Y^{*}\left(\tau_{2}(X)\right),
$$

where $\mathcal{P}^{2}(\mathfrak{g}(1))$ is the space of polynomials on $\mathfrak{g}(1)$ of degree 2. By Proposition 6.8, we know that if $\left.\tilde{\tau}_{2}\right|_{V(\nu) *}$ is not identically zero, then $V(\nu)$ is a special constituent of $\mathfrak{l} \otimes \mathfrak{z}(\mathfrak{n})$. We now show that the converse of Proposition 6.8 also holds for special constituents $V(\nu)$ of type 1a or type 2 . If $l \in L$ and $Z \in \mathfrak{l}$, then we denote the action of the group and its Lie algebra on $X_{\alpha} \otimes X_{\gamma_{j}}$ by $l \cdot\left(X_{\alpha} \otimes X_{\gamma_{j}}\right)$ and $Z \cdot\left(X_{\alpha} \otimes X_{\gamma_{j}}\right)$, respectively.

Proposition 7.5. If $V(\mu+\epsilon)$ is a special constituent of $\mathfrak{l} \otimes \mathfrak{z}(\mathfrak{n})$ of type 1 a or type 2, then the following hold:

(1) The vector $\tau_{2}\left(X_{\mu}+X_{\epsilon}\right)$ is a highest weight vector for $V(\mu+\epsilon)$.

(2) The L-intertwining operator $\left.\tilde{\tau}_{2}\right|_{V(\mu+\epsilon) *}$ is not identically zero.

Proof. We have shown in the proof for Proposition 7.3 that $\tau_{2}\left(X_{\mu}+X_{\epsilon}\right) \neq 0$. Moreover, Lemma 7.1 gives that $\tau_{2}\left(X_{\mu}+X_{\epsilon}\right)=a_{\mu, \epsilon} \operatorname{ad}\left(X_{\mu}\right) \operatorname{ad}\left(X_{\epsilon}\right) \omega$ with $a_{\mu, \epsilon}=$ $1+\delta_{\mu, \epsilon}$. For $l \in L$, we have $l \cdot \omega=\omega$ (see Lemma 3.2), and so

$$
l \cdot \tau_{2}\left(X_{\mu}+X_{\epsilon}\right)=a_{\mu, \epsilon}\left(\operatorname{ad}\left(\operatorname{Ad}(l) X_{\mu}\right) \operatorname{ad}\left(\operatorname{Ad}(l) X_{\epsilon}\right) \otimes \mathrm{Id}\right) \omega .
$$

By replacing $l$ by $\exp (t Z)$ with $Z \in \mathfrak{l}$, differentiating, and setting $t=0$, we obtain

$$
Z \cdot \tau_{2}\left(X_{\mu}+X_{\epsilon}\right)=a_{\mu, \epsilon}\left(\left(\operatorname{ad}\left(\left[Z, X_{\mu}\right]\right) \operatorname{ad}\left(X_{\epsilon}\right)+\operatorname{ad}\left(X_{\mu}\right) \operatorname{ad}\left(\left[Z, X_{\epsilon}\right]\right)\right) \otimes \operatorname{Id}\right) \omega
$$

In particular, if $Z=H \in \mathfrak{h}$ in (17.6), then

$$
H \cdot \tau_{2}\left(X_{\mu}+X_{\epsilon}\right)=(\mu+\epsilon)(H) \tau_{2}\left(X_{\mu}+X_{\epsilon}\right) .
$$

Therefore $\tau_{2}\left(X_{\mu}+X_{\epsilon}\right)$ is a weight vector with weight $\mu+\epsilon$. To show that $\tau_{2}\left(X_{\mu}+X_{\epsilon}\right)$ is a highest weight vector, we replace $Z$ in (17.6) by $X_{\alpha}$ with $\alpha \in \Delta^{+}(\mathfrak{l})$. Since $\mu$ is the highest weight for $\mathfrak{g}(1)$, we have

$$
X_{\alpha} \cdot \tau_{2}\left(X_{\mu}+X_{\epsilon}\right)=a_{\mu, \epsilon}\left(\operatorname{ad}\left(X_{\mu}\right) \operatorname{ad}\left(\left[X_{\alpha}, X_{\epsilon}\right]\right) \otimes \mathrm{Id}\right) \omega .
$$

If $\mu+\epsilon$ is of type 2 , then, as $\epsilon=\mu$ in the case, clearly $X_{\alpha} \cdot \tau_{2}\left(X_{\mu}+X_{\epsilon}\right)=0$. The case that $\mu+\epsilon$ is of type 1a follows from Lemma 6.25.

To prove the second statement, it is enough to show that there exist $Y^{*} \in$ $V(\mu+\epsilon)^{*}$ and $X \in \mathfrak{g}(1)$ so that $\tilde{\tau}_{2}\left(Y^{*}\right)(X) \neq 0$. Let $Y_{l}^{*}$ be a lowest weight vector for $V(\mu+\epsilon)^{*}$. Observe that if $Y_{h}$ is a highest weight vector for $V(\mu+\epsilon)$, then $Y_{l}^{*}\left(Y_{h}\right) \neq 0$. Since $\tau_{2}\left(X_{\mu}+X_{\epsilon}\right)$ is a highest weight vector for $V(\mu+\epsilon)$, we have

$$
\left.\tilde{\tau}_{2}\right|_{V(\mu+\epsilon)^{*}}\left(Y_{l}^{*}\right)\left(X_{\mu}+X_{\epsilon}\right)=Y_{l}^{*}\left(\tau_{2}\left(X_{\mu}+X_{\epsilon}\right)\right) \neq 0 \text {. }
$$


7.2. The $\left.\Omega_{2}\right|_{V(\mu+\epsilon)^{*}}$ systems. Proposition 7.5 shows that the $L$-intertwining operator $\left.\tilde{\tau}_{2}\right|_{V(\mu+\epsilon)^{*}}$ is not identically zero when $V(\mu+\epsilon)$ is a special constituent of $\mathfrak{l} \otimes \mathfrak{z}(\mathfrak{n})$ of type 1a or type 2 . We thus construct the $\left.\Omega_{2}\right|_{V(\mu+\epsilon)^{*}}$ system corresponding to irreducible constituents $V(\mu+\epsilon)$ of type 1a or type 2 . Here it may be helpful to recall some notation introduced in Subsection 6.3. For any ad $(\mathfrak{h})$-invariant subspace $W \subset \mathfrak{g}$ and any weight $\nu \in \mathfrak{h}^{*}$, we write

$$
\Delta_{\nu}(W)=\{\alpha \in \Delta(W) \mid \nu-\alpha \in \Delta\}
$$

When $V(\mu+\epsilon)$ is a special constituent of $\mathfrak{l} \otimes \mathfrak{z}(\mathfrak{n})$, we write

$$
\theta(\beta)=(\mu+\epsilon)-\beta
$$

As indicated in Subsection 3.1, the $L$-intertwining operator $\left.\tilde{\tau}_{2}\right|_{V(\mu+\epsilon)^{*}}$ yields a system of differential operators. We have denoted such operators by $\Omega_{2}\left(Y^{*}\right)=$ $\left.\Omega_{2}\right|_{V(\mu+\epsilon)^{*}}\left(Y^{*}\right)$ with $Y^{*} \in V(\mu+\epsilon)^{*}$, where $\left.\Omega_{2}\right|_{V(\mu+\epsilon)^{*}}: V(\mu+\epsilon)^{*} \rightarrow \mathbb{D}\left(\mathcal{L}_{s}\right)^{\overline{\mathfrak{n}}}$ is $\mathcal{U}(\mathfrak{l})$-equivariant. Because of such equivariance, the system is totally determined, once $\Omega_{2}\left(Y_{l}^{*}\right)$ is constructed, where $Y_{l}^{*}$ is a lowest weight vector in $V(\mu+\epsilon)^{*}$.

The first step is to explicitly describe $Y_{l}^{*} \in V(\mu+\epsilon)^{*}$. Observe that we have a non-zero map

$$
\begin{aligned}
\bar{\tau}_{2}: \mathfrak{g}(-1) & \rightarrow \mathfrak{l} \otimes \mathfrak{z}(\overline{\mathfrak{n}}), \\
\bar{X} & \mapsto \frac{1}{2}\left(\operatorname{ad}(\bar{X})^{2} \otimes \mathrm{Id}\right) \bar{\omega}
\end{aligned}
$$

with $\bar{\omega}=\sum_{\gamma_{t} \in \Delta(\mathfrak{z}(\mathfrak{n}))} X_{\gamma_{t}} \otimes X_{-\gamma_{t}}$. One checks, as in the proofs for Lemma 3.3 and Proposition 7.3, that $\bar{\tau}_{2}$ is a non-zero $L$-equivariant map. Moreover, if $V(\mu+\epsilon)$ is a special constituent of type $1 \mathrm{a}$ or type 2 , then

$$
\bar{\tau}_{2}\left(X_{-\mu}+X_{-\epsilon}\right)=a_{\mu, \epsilon}\left(\operatorname{ad}\left(X_{-\mu}\right) \operatorname{ad}\left(X_{-\epsilon}\right) \otimes \mathrm{Id}\right) \bar{\omega}
$$

with $a_{\mu, \epsilon}=1+\delta_{\mu, \epsilon}$. Arguing as in Proposition 7.5, we can show that $\bar{\tau}_{2}\left(X_{-\mu}+X_{-\epsilon}\right)$ is a lowest weight vector for $V(\mu+\epsilon)^{*}$ with lowest weight $-\mu-\epsilon$. Thus,

$$
Y_{l}^{*}=\left(\operatorname{ad}\left(X_{-\mu}\right) \operatorname{ad}\left(X_{-\epsilon}\right) \otimes \operatorname{Id}\right) \bar{\omega}=\sum_{\gamma_{t} \in \Delta_{\mu+\epsilon}(\mathfrak{z}(\mathfrak{n}))} N_{-\mu, \gamma_{t}-\epsilon} N_{-\epsilon, \gamma_{t}} X_{-\theta\left(\gamma_{t}\right)} \otimes X_{-\gamma_{t}}
$$

is a lowest weight vector for $V(\mu+\epsilon)^{*}$. Observe that, by Lemma 6.31, we have $\gamma_{t}-\epsilon \in \Delta$ for $\gamma_{t} \in \Delta_{\mu+\epsilon}(\mathfrak{z}(\mathfrak{n}))$. Then, by (7.7), we have

$$
\begin{aligned}
& Y_{l}^{*}\left(\tau_{2}(X)\right)=\frac{1}{2} \sum_{\substack{\left.\gamma_{t} \in \Delta_{\mu+\epsilon}(\mathfrak{z}(\mathfrak{n})) \\
\gamma_{j} \in \Delta(\mathfrak{z} \mathfrak{n})\right)}} N_{-\mu, \gamma_{t}-\epsilon} N_{-\epsilon, \gamma_{t}} \kappa\left(X_{-\theta\left(\gamma_{t}\right)}, \operatorname{ad}(X)^{2} X_{-\gamma_{j}}\right) \kappa\left(X_{-\gamma_{t}}, X_{\gamma_{j}}\right) \\
& (7.8) \quad=\frac{1}{2} \sum_{\gamma_{t} \in \Delta_{\mu+\epsilon}(\mathfrak{z}(\mathfrak{n}))} N_{-\mu, \gamma_{t}-\epsilon} N_{-\epsilon, \gamma_{t}} \kappa\left(X_{-\theta\left(\gamma_{t}\right)}, \operatorname{ad}(X)^{2} X_{-\gamma_{t}}\right) .
\end{aligned}
$$


Write $X=\sum_{\alpha \in \Delta(\mathfrak{g}(1))} \eta_{\alpha} X_{\alpha}$ and let $\gamma_{t} \in \Delta_{\mu+\epsilon}(\mathfrak{z}(\mathfrak{n}))$. Then,

$$
\begin{aligned}
\kappa\left(X_{-\theta\left(\gamma_{t}\right)}, \operatorname{ad}(X)^{2} X_{-\gamma_{t}}\right) & =\sum_{\alpha, \beta \in \Delta(\mathfrak{g}(1))} \eta_{\alpha} \eta_{\beta} \kappa\left(X_{-\theta\left(\gamma_{t}\right)},\left[X_{\beta},\left[X_{\alpha}, X_{-\gamma_{t}}\right]\right]\right) \\
& =\sum_{\alpha, \beta \in \Delta(\mathfrak{g}(1))} \eta_{\alpha} \eta_{\beta} \kappa\left(\left[X_{-\theta\left(\gamma_{t}\right)}, X_{\beta}\right],\left[X_{\alpha}, X_{-\gamma_{t}}\right]\right) \\
& =\sum_{\substack{\alpha \in \Delta_{\gamma_{t}}(\mathfrak{g}(1)) \\
\beta \in \Delta_{\theta\left(\gamma_{t}\right)}(\mathfrak{g}(1))}} \eta_{\alpha} \eta_{\beta} N_{\alpha,-\gamma_{t}} N_{-\theta\left(\gamma_{t}\right), \beta} \kappa\left(X_{\beta-\theta\left(\gamma_{t}\right)}, X_{\alpha-\gamma_{t}}\right) .
\end{aligned}
$$

Observe that, by Lemma 6.34 and Lemma 6.35, the sets $\Delta_{\gamma_{t}}(\mathfrak{g}(1))$ and $\Delta_{\theta\left(\gamma_{t}\right)}(\mathfrak{g}(1))$ are non-empty. By the normalization (H3) in Section 5, if $\kappa\left(X_{\beta-\theta\left(\gamma_{t}\right)}, X_{\alpha-\gamma_{t}}\right) \neq 0$, then $\beta-\theta\left(\gamma_{t}\right)=\gamma_{t}-\alpha$. Thus $\kappa\left(X_{\beta-\theta\left(\gamma_{t}\right)}, X_{\alpha-\gamma_{t}}\right)=0$ unless $\beta=(\mu+\epsilon)-\alpha=\theta(\alpha)$. Therefore,

$$
\begin{aligned}
\kappa\left(X_{-\theta\left(\gamma_{t}\right)}, \operatorname{ad}(X)^{2} X_{-\gamma_{t}}\right) & =\sum_{\substack{\alpha \in \Delta_{\gamma_{t}}(\mathfrak{g}(1)) \\
\beta \in \Delta_{\theta\left(\gamma_{t}\right)}(\mathfrak{g}(1))}} \eta_{\alpha} \eta_{\beta} N_{\alpha,-\gamma_{t}} N_{-\theta\left(\gamma_{t}\right), \beta} \kappa\left(X_{\beta-\theta\left(\gamma_{t}\right)}, X_{\alpha-\gamma_{t}}\right) \\
& =\sum_{\alpha \in \Delta_{\gamma t}(\mathfrak{g}(1)) \cap \Delta_{\mu+\epsilon}(\mathfrak{g}(1))} N_{\alpha,-\gamma_{t}} N_{-\theta\left(\gamma_{t}\right), \theta(\alpha)} \eta_{\alpha} \eta_{\theta(\alpha)} \\
& =\sum_{\alpha \in \Delta_{\mu+\epsilon}(\mathfrak{g}(1))} N_{\alpha,-\gamma_{t}} N_{-\theta\left(\gamma_{t}\right), \theta(\alpha)} \kappa\left(X, X_{-\alpha}\right) \kappa\left(X, X_{-\theta(\alpha)}\right) .
\end{aligned}
$$

Lemma 6.34 is used in line three to show that $\Delta_{\gamma_{t}}(\mathfrak{g}(1)) \cap \Delta_{\mu+\epsilon}(\mathfrak{g}(1))=\Delta_{\mu+\epsilon}(\mathfrak{g}(1))$. Hence, by (7.8) and (7.9), $\left.\tilde{\tau}_{2}\right|_{V(\mu+\epsilon)^{*}}\left(Y_{l}^{*}\right)(X)=Y_{l}^{*}\left(\tau_{2}(X)\right)$ is

$$
\begin{aligned}
& \left.\tilde{\tau}_{2}\right|_{V(\mu+\epsilon)^{*}}\left(Y_{l}^{*}\right)(X) \\
& =\frac{1}{2} \sum_{\substack{\alpha \in \Delta_{\mu+\epsilon}(\mathfrak{g}(1)) \\
\gamma_{t} \in \Delta_{\mu+\epsilon}(\mathfrak{z}(\mathfrak{n}))}}\left(N_{-\mu, \gamma_{t}-\epsilon} N_{-\epsilon, \gamma_{t}}\right)\left(N_{\alpha,-\gamma_{t}} N_{-\theta\left(\gamma_{t}\right), \theta(\alpha)}\right) \kappa\left(X, X_{-\alpha}\right) \kappa\left(X, X_{-\theta(\alpha)}\right) .
\end{aligned}
$$

Now, via the composition of maps

$$
V(\mu+\epsilon)^{*} \stackrel{\left.\tilde{\tau}_{2}\right|_{V(\mu+\epsilon)^{*}}}{\rightarrow} \mathcal{P}^{2}(\mathfrak{g}(1)) \rightarrow \operatorname{Sym}^{2}(\mathfrak{g}(-1)) \stackrel{\sigma}{\hookrightarrow} \mathcal{U}(\overline{\mathfrak{n}}) \stackrel{R}{\rightarrow} \mathbb{D}\left(\mathcal{L}_{s}\right)^{\overline{\mathfrak{n}}},
$$

for $Y_{l}^{*} \in V(\mu+\epsilon)^{*}$, the second order differential operator $\Omega_{2}\left(Y_{l}^{*}\right) \in \mathbb{D}\left(\mathcal{L}_{s}\right)^{\overline{\mathfrak{n}}}$ is given by

$$
\Omega_{2}\left(Y_{l}^{*}\right)=\frac{1}{2} \sum_{\substack{\alpha \in \Delta_{\mu+\epsilon}(\mathfrak{g}(1)) \\ \gamma_{t} \in \Delta_{\mu+\epsilon}(\mathfrak{z}(\mathfrak{n}))}}\left(N_{-\mu, \gamma_{t}-\epsilon} N_{-\epsilon, \gamma_{t}}\right)\left(N_{\alpha,-\gamma_{t}} N_{-\theta\left(\gamma_{t}\right), \theta(\alpha)}\right) \sigma\left(R\left(X_{-\alpha}\right) R\left(X_{-\theta(\alpha)}\right)\right),
$$

where $\sigma(a b)=(1 / 2)(a b+b a)$. As the special constituent $V(\mu+\epsilon)$ is assumed to be of type 1 a or type 2 , we have $-\alpha-\theta(\alpha)=-(\mu+\epsilon) \notin \Delta$. Thus symmetrization is unnecessary. Therefore we obtain

$$
\Omega_{2}\left(Y_{l}^{*}\right)=\frac{1}{2} \sum_{\substack{\alpha \in \Delta_{\mu+\epsilon}(\mathfrak{g}(1)) \\ \gamma_{t} \in \Delta_{\mu+\epsilon}(\mathfrak{z}(\mathfrak{n}))}}\left(N_{-\mu, \gamma_{t}-\epsilon} N_{-\epsilon, \gamma_{t}}\right)\left(N_{\alpha,-\gamma_{t}} N_{-\theta\left(\gamma_{t}\right), \theta(\alpha)}\right) R\left(X_{-\alpha}\right) R\left(X_{-\theta(\alpha)}\right) .
$$


7.3. Special values of the $\left.\Omega_{2}\right|_{V(\mu+\epsilon)^{*}}$ systems. Now we determine the special values of the line bundle $\mathcal{L}_{s}$ for which the $\left.\Omega_{2}\right|_{V(\mu+\epsilon)^{*}}$ system is conformally invariant, under the assumption that $V(\mu+\epsilon)$ is a special constituent of type 1a or type 2 .

Choose a basis $\left\{Y_{1}^{*}, \ldots, Y_{n}^{*}\right\}$ for $V(\mu+\epsilon)^{*}$. To show that the list of differential operators $\Omega_{2}\left(Y_{1}^{*}\right), \ldots, \Omega_{2}\left(Y_{n}^{*}\right)$ is conformally invariant on the bundle $\mathcal{L}_{s}$, we need to prove that in $\mathbb{D}\left(\mathcal{L}_{s}\right)^{\overline{\mathfrak{n}}}$,

$$
\left[\pi_{s}(X), \Omega_{2}\left(Y_{i}^{*}\right)\right] \in \operatorname{span}_{C^{\infty}\left(\bar{N}_{0}\right)}\left\{\Omega_{2}\left(Y_{1}^{*}\right), \ldots, \Omega_{2}\left(Y_{n}^{*}\right)\right\}
$$

for all $X \in \mathfrak{g}$ and all $i$. By Proposition 2.16, (7.11) holds if

$$
\left[\pi_{s}(X), \Omega_{2}\left(Y_{i}^{*}\right)\right]_{e} \in \operatorname{span}_{\mathbb{C}}\left\{\Omega_{2}\left(Y_{1}^{*}\right)_{e}, \cdots, \Omega_{2}\left(Y_{n}^{*}\right)_{e}\right\}
$$

holds for all $X \in \mathfrak{g}$ and all $i$. Here, for $D \in \mathbb{D}\left(\mathcal{L}_{s}\right), D_{\bar{n}}$ denotes the linear functional $f \mapsto(D \bullet f)(\bar{n})$ for $f \in C^{\infty}\left(\bar{N}_{0}, \mathbb{C}_{\chi^{s}}\right)$. We show that a simplification of (7.12) implies (7.11).

Proposition 7.13. Let $V(\mu+\epsilon)^{*}$ be the dual module of a special constituent $V(\mu+\epsilon)$ of $\mathfrak{l} \otimes \mathfrak{z}(\mathfrak{n})$ with respect to the Killing form. Suppose that the operator $\left.\Omega_{2}\right|_{V(\mu+\epsilon)^{*}}$ : $V(\mu+\epsilon)^{*} \rightarrow \mathbb{D}\left(\mathcal{L}_{s}\right)^{\overline{\mathfrak{n}}}$ is non-zero. If $X_{h}$ is a highest weight vector for $\mathfrak{g}(1)$ and if we have

$$
\left[\pi_{s}\left(X_{h}\right), \Omega_{2}\left(Y_{l}^{*}\right)\right]_{e} \in \operatorname{span}_{\mathbb{C}}\left\{\Omega_{2}\left(Y_{1}^{*}\right)_{e}, \cdots, \Omega_{2}\left(Y_{n}^{*}\right)_{e}\right\}
$$

for a lowest weight vector $Y_{l}^{*}$ and a basis $\left\{Y_{1}^{*}, \ldots, Y_{n}^{*}\right\}$ for $V(\mu+\epsilon)^{*}$, then the $\left.\Omega_{2}\right|_{V(\mu+\epsilon)^{*}}$ system is a conformally invariant system.

Proof. By Remark 3.8, the $\left.\Omega_{k}\right|_{V(\mu+\epsilon)^{*}}$ system satisfies the condition (S1) of Definition 2.3. We need to prove that (17.12) holds for all $X \in \mathfrak{g}=\overline{\mathfrak{n}} \oplus \mathfrak{l} \oplus \mathfrak{n}$. Note that, by definition, we have $\Omega_{2}\left(Y_{i}^{*}\right) \in \mathbb{D}\left(\mathcal{L}_{s}\right)^{\overline{\mathfrak{n}}}$. Hence (17.12) holds for $X \in \overline{\mathfrak{n}}$ trivially. The $L_{0}$-equivariance of $\left.\Omega_{2}\right|_{V(\mu+\epsilon)^{*}}$ shows that (7.12) holds for $X \in \mathfrak{l}$. Furthermore, Lemma 3.12 established (17.12) when $X \in \mathfrak{g}(1)$. Now we handle the case when $X \in \mathfrak{z}(\mathfrak{n})$.

If $X \in \mathfrak{z}(\mathfrak{n})$, then, since $\mathfrak{z}(\mathfrak{n})=[\mathfrak{g}(1), \mathfrak{g}(1)]$, it is of the form $X=\left[X_{1}, X_{2}\right]$ for some $X_{1}, X_{2} \in \mathfrak{g}(1)$. Then, by the Jacobi identity, we have

$$
\left[\pi_{s}(X), \Omega_{2}\left(Y_{i}^{*}\right)\right]=\left[\pi_{s}\left(X_{1}\right),\left[\pi_{s}\left(X_{2}\right), \Omega_{2}\left(Y_{i}^{*}\right)\right]\right]-\left[\pi_{s}\left(X_{2}\right),\left[\pi_{s}\left(X_{1}\right), \Omega_{2}\left(Y_{i}^{*}\right)\right]\right] .
$$

By (2.9), we have $\pi_{s}\left(X_{j}\right)_{e}=0$ for $j=1,2$. It follows from Lemma 3.12 that for $j=1,2$ and all $i$, we have

$$
\left[\pi_{s}\left(X_{j}\right), \Omega_{2}\left(Y^{*}\right)\right]_{e} \in \operatorname{span}_{\mathbb{C}}\left\{\Omega_{2}\left(Y_{1}^{*}\right)_{e}, \ldots, \Omega_{2}\left(Y_{n}^{*}\right)_{e}\right\} .
$$

Therefore, by Lemma 3.9.

$$
\begin{aligned}
{\left[\pi_{s}(X), \Omega_{2}\left(Y_{i}^{*}\right)\right]_{e} } & =\left[\pi_{s}\left(X_{1}\right),\left[\pi_{s}\left(X_{2}\right), \Omega_{2}\left(Y_{i}^{*}\right)\right]\right]_{e}-\left[\pi_{s}\left(X_{2}\right),\left[\pi_{s}\left(X_{1}\right), \Omega_{2}\left(Y_{i}^{*}\right)\right]\right]_{e} \\
& \in \operatorname{span}_{\mathbb{C}}\left\{\Omega_{2}\left(Y_{1}^{*}\right)_{e}, \ldots, \Omega_{2}\left(Y_{k}^{*}\right)_{e}\right\} .
\end{aligned}
$$

Proposition 7.14. If $\mu$ is the highest weight for $\mathfrak{g}(1)$ and $\alpha, \beta \in \Delta(\mathfrak{g}(1))$, then

$$
\begin{aligned}
& {\left[\pi_{s}\left(X_{\mu}\right), R\left(X_{-\alpha}\right) R\left(X_{-\beta}\right)\right]_{e}} \\
& =R\left(\left[\left[X_{\mu}, X_{-\alpha}\right], X_{-\beta}\right]\right)_{e}-s \lambda_{\mathfrak{q}}\left(\left[X_{\mu}, X_{-\alpha}\right]\right) R\left(X_{-\beta}\right)_{e}-s \lambda_{\mathfrak{q}}\left(\left[X_{\mu}, X_{-\beta}\right]\right) R\left(X_{-\alpha}\right)_{e} .
\end{aligned}
$$

Proof. This simply follows by substituting $Y=X_{\mu}, X_{1}=X_{-\alpha}$, and $X_{2}=X_{-\beta}$ in Proposition 2.17, and evaluating at $\bar{n}=e$. 
If $V(\mu+\epsilon)$ is a special constituent of $\mathfrak{l} \otimes \mathfrak{z}(\mathfrak{n})$ of type 1a or type 2 , then we write

$$
C(\mu, \epsilon):=\sum_{\gamma_{t} \in \Delta_{\mu+\epsilon}(\mathfrak{z}(\mathfrak{n}))} N_{\mu, \epsilon-\gamma_{t}} N_{-\mu, \gamma_{t}-\epsilon} N_{\epsilon,-\gamma_{t}} N_{-\epsilon, \gamma_{t}} .
$$

By Lemma 6.36, we have $C(\mu, \epsilon) \neq 0$.

Theorem 7.16. Let $\mathfrak{g}$ be a complex simple Lie algebra and $\mathfrak{q}$ be a maximal parabolic subalgebra of quasi-Heisenberg type listed in (4.9) or (4.10). If $Y_{l}^{*}$ is the lowest weight vector defined in (7.7) for the dual module $V(\mu+\epsilon)^{*}$ of a special constituent $V(\mu+\epsilon)$ of type 1a or type 2, and if $\alpha_{\mathfrak{q}}$ is the simple root that determines $\mathfrak{q}$, then the following hold:

(1) If $V(\mu+\epsilon)$ is of type 1a, then

$$
\left[\pi_{s}\left(X_{\mu}\right), \Omega_{2}\left(Y_{l}^{*}\right)\right]_{e}=-\frac{\left\|\alpha_{\mathfrak{q}}\right\|^{2}}{2} C(\mu, \epsilon)\left(s-s_{2}\right) R\left(X_{-\epsilon}\right)_{e},
$$

with $s_{2}=\frac{\left|\Delta_{\mu+\epsilon}(\mathfrak{g}(1))\right|}{2}-1$, where $\left|\Delta_{\mu+\epsilon}(\mathfrak{g}(1))\right|$ is the number of elements in $\Delta_{\mu+\epsilon}(\mathfrak{g}(1))$.

(2) If $V(\mu+\epsilon)$ is of type 2, then

$$
\left[\pi_{s}\left(X_{\mu}\right), \Omega_{2}\left(Y_{l}^{*}\right)\right]_{e}=-\frac{\left\|\alpha_{\mathfrak{q}}\right\|^{2}}{2} C(\mu, \mu)(s+1) R\left(X_{-\mu}\right)_{e} .
$$

Proof. We start by showing that (17.17) holds. It follows from (7.10) that

$$
\begin{aligned}
& {\left[\pi_{s}\left(X_{\mu}\right), \Omega_{2}\left(Y_{l}^{*}\right)\right]_{e}} \\
& =\frac{1}{2} \sum_{\substack{\alpha \in \Delta_{\mu+\epsilon}(\mathfrak{g}(1)) \\
\gamma_{t} \in \Delta_{\mu+\epsilon}(\mathfrak{z}(\mathfrak{n}))}}\left(N_{-\mu, \gamma_{t}-\epsilon} N_{-\epsilon, \gamma_{t}}\right)\left(N_{\alpha,-\gamma_{t}} N_{-\theta\left(\gamma_{t}\right), \theta(\alpha)}\right)\left[\pi_{s}\left(X_{\mu}\right), R\left(X_{-\alpha}\right) R\left(X_{-\theta(\alpha)}\right)\right]_{e} .
\end{aligned}
$$

We use Proposition 7.14 to compute $\left[\pi_{s}\left(X_{\mu}\right), R\left(X_{-\alpha}\right) R\left(X_{-\theta(\alpha)}\right)\right]_{e}$. This is

$$
\begin{aligned}
{\left[\pi_{s}\left(X_{\mu}\right), R\left(X_{-\alpha}\right) R\left(X_{-\theta(\alpha)}\right)\right]_{e} } \\
\quad=R\left(\left[\left[X_{\mu}, X_{-\alpha}\right], X_{-\theta(\alpha)}\right]\right)_{e}-s \lambda_{\mathfrak{q}}\left(\left[X_{\mu}, X_{-\alpha}\right]\right) R\left(X_{-\theta(\alpha)}\right)_{e} \\
\quad-s \lambda_{\mathfrak{q}}\left(\left[X_{\mu}, X_{-\theta(\alpha)}\right]\right) R\left(X_{-\alpha}\right)_{e} .
\end{aligned}
$$

We consider the contributions from each term in (7.19) separately. Recall here that, as we defined in Section 4.2, our parabolic subalgebra $\mathfrak{q}$ is parametrized by the simple root $\alpha_{\mathfrak{q}} \in \Pi$ and that $\lambda_{\mathfrak{q}}$ is the fundamental weight for $\alpha_{\mathfrak{q}}$.

First we study the contribution from the second term. It is

$$
\begin{aligned}
T_{2}= & -\frac{s}{2} \sum_{\substack{\alpha \in \Delta_{\mu+\epsilon}(\mathfrak{g}(1)) \\
\gamma_{t} \in \Delta_{\mu+\epsilon}(\mathfrak{z}(\mathfrak{n}))}}\left(N_{-\mu, \gamma_{t}-\epsilon} N_{-\epsilon, \gamma_{t}}\right)\left(N_{\alpha,-\gamma_{t}} N_{-\theta\left(\gamma_{t}\right), \theta(\alpha)}\right) \\
& \times \lambda_{\mathfrak{q}}\left(\left[X_{\mu}, X_{-\alpha}\right]\right) R\left(X_{-\theta(\alpha)}\right)_{e} .
\end{aligned}
$$

As $\mathfrak{g}(1)$ is the 1-eigenspace of $\operatorname{ad}\left(H_{\mathfrak{q}}\right)$ with $H_{\mathfrak{q}}$ defined in (4.4), the set $\Delta(\mathfrak{g}(1))$ is $\Delta(\mathfrak{g}(1))=\left\{\beta \in \Delta \mid 2\left\langle\lambda_{\mathfrak{q}}, \beta\right\rangle /\left\|\alpha_{\mathfrak{q}}\right\|^{2}=1\right\}$. Therefore, by the normalization (H4) in Section [5 for $\beta \in \Delta(\mathfrak{g}(1))$, we have $\lambda_{\mathfrak{q}}\left(H_{\beta}\right)=\left\langle\lambda_{\mathfrak{q}}, \beta\right\rangle=\left\|\alpha_{\mathfrak{q}}\right\|^{2} / 2$. Thus,

$$
\lambda_{\mathfrak{q}}\left(\left[X_{\mu}, X_{-\alpha}\right]\right)=\frac{\left\|\alpha_{\mathfrak{q}}\right\|^{2}}{2} \delta_{\alpha, \mu}
$$


with $\delta_{\alpha, \mu}$ the Kronecker delta. So the contribution from this term is

$$
\begin{aligned}
T_{2} & =-\frac{s}{2} \sum_{\substack{\left.\alpha \in \Delta_{\mu+\epsilon}(\mathfrak{g}(1)) \\
\gamma_{t} \in \Delta_{\mu+\epsilon}(\mathfrak{z})\right)}}\left(N_{-\mu, \gamma_{t}-\epsilon} N_{-\epsilon, \gamma_{t}}\right)\left(N_{\alpha,-\gamma_{t}} N_{-\theta\left(\gamma_{t}\right), \theta(\alpha)}\right) \lambda_{\mathfrak{q}}\left(\left[X_{\mu}, X_{-\alpha}\right]\right) R\left(X_{-\theta(\alpha)}\right)_{e} \\
& =-\frac{s\left\|\alpha_{\mathfrak{q}}\right\|^{2}}{4} \sum_{\substack{\gamma_{t} \in \Delta_{\mu+\epsilon}(\mathfrak{z}(\mathfrak{n}))\\
}}\left(N_{-\mu, \gamma_{t}-\epsilon} N_{-\epsilon, \gamma_{t}}\right)\left(N_{\mu,-\gamma_{t}} N_{-\theta\left(\gamma_{t}\right), \theta(\mu)}\right) R\left(X_{-\theta(\mu)}\right)_{e} \\
& =-\frac{s\left\|\alpha_{\mathfrak{q}}\right\|^{2}}{4} \sum_{\gamma_{t} \in \Delta_{\mu+\epsilon}(\mathfrak{z}(\mathfrak{n}))}\left(N_{-\mu, \gamma_{t}-\epsilon} N_{-\epsilon, \gamma_{t}}\right)\left(N_{\mu,-\gamma_{t}} N_{\epsilon, \mu-\gamma_{t}}\right) R\left(X_{-\epsilon}\right)_{e} .
\end{aligned}
$$

We showed in Lemma 6.30 that $N_{\mu,-\gamma_{t}} N_{\epsilon, \mu-\gamma_{t}}=N_{\mu, \epsilon-\gamma_{t}} N_{\epsilon,-\gamma_{t}}$. Hence,

$$
\begin{aligned}
T_{2} & =-\frac{s\left\|\alpha_{\mathfrak{q}}\right\|^{2}}{4} \sum_{\gamma_{t} \in \Delta_{\mu+\epsilon}(\mathfrak{z}(\mathfrak{n}))}\left(N_{-\mu, \gamma_{t}-\epsilon} N_{-\epsilon, \gamma_{t}}\right)\left(N_{\mu,-\gamma_{t}} N_{\epsilon, \mu-\gamma_{t}}\right) R\left(X_{-\epsilon}\right)_{e} \\
& =-\frac{s\left\|\alpha_{\mathfrak{q}}\right\|^{2}}{4} \sum_{\gamma_{t} \in \Delta_{\mu+\epsilon}(\mathfrak{z}(\mathfrak{n}))}\left(N_{-\mu, \gamma_{t}-\epsilon} N_{-\epsilon, \gamma_{t}}\right)\left(N_{\mu, \epsilon-\gamma_{t}} N_{\epsilon,-\gamma_{t}}\right) R\left(X_{-\epsilon}\right)_{e} \\
& =-\frac{s\left\|\alpha_{\mathfrak{q}}\right\|^{2}}{4} \sum_{\gamma_{t} \in \Delta_{\mu+\epsilon}(\mathfrak{z}(\mathfrak{n}))}\left(N_{\mu, \epsilon-\gamma_{t}} N_{-\mu, \gamma_{t}-\epsilon} N_{\epsilon,-\gamma_{t}} N_{-\epsilon, \gamma_{t}}\right) R\left(X_{-\epsilon}\right)_{e} \\
& =-\frac{s\left\|\alpha_{\mathfrak{q}}\right\|^{2}}{4} C(\mu, \epsilon) R\left(X_{-\epsilon}\right)_{e} .
\end{aligned}
$$

The same argument shows that the contribution from the third term is

$$
\begin{aligned}
T_{3} & =-\frac{s}{2} \sum_{\substack{\alpha \in \Delta_{\mu+\epsilon}(\mathfrak{g}(1)) \\
\gamma_{t} \in \Delta_{\mu+\epsilon}(\mathfrak{z}(\mathfrak{n}))}}\left(N_{-\mu, \gamma_{t}-\epsilon} N_{-\epsilon, \gamma_{t}}\right)\left(N_{\alpha,-\gamma_{t}} N_{-\theta\left(\gamma_{t}\right), \theta(\alpha)}\right) \lambda_{\mathfrak{q}}\left(\left[X_{\mu}, X_{-\theta(\alpha)}\right]\right) R\left(X_{-\alpha}\right)_{e} \\
& =-\frac{s\left\|\alpha_{\mathfrak{q}}\right\|^{2}}{4} C(\mu, \epsilon) R\left(X_{-\epsilon}\right)_{e} .
\end{aligned}
$$

Now we consider the contribution from the first term. It is

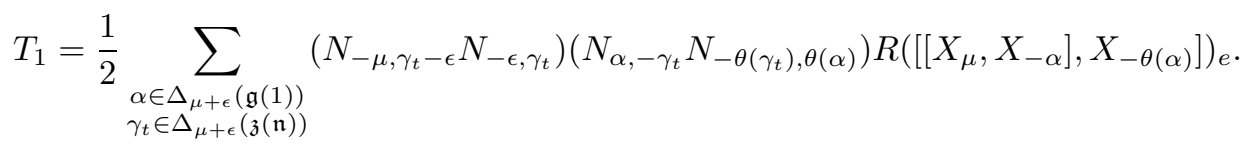

We claim that if $\alpha=\epsilon$ or $\mu$, then $\left[\left[X_{\mu}, X_{-\alpha}\right], X_{-\theta(\alpha)}\right]=0$, where $\theta(\alpha)$ denotes $\theta(\alpha)=(\mu+\epsilon)-\alpha$. If $\alpha=\epsilon$, then, by Remark [6.21, $\left[X_{\mu}, X_{-\alpha}\right]=\left[X_{\mu}, X_{-\epsilon}\right]=0$. If $\alpha=\mu$, then

$$
\left[\left[X_{\mu}, X_{-\mu}\right], X_{-\theta(\mu)}\right]=\left[\left[X_{\mu}, X_{-\mu}\right], X_{-\epsilon}\right]=\epsilon\left(H_{\mu}\right) X_{-\epsilon}=0
$$


Note that Remark 6.21 is applied to obtain $\epsilon\left(H_{\mu}\right)=\langle\epsilon, \mu\rangle=0$. Moreover, by Remark 6.32, we have $\Delta_{\mu+\epsilon}(\mathfrak{g}(1)) \backslash\{\mu, \epsilon\} \neq \emptyset$. The contribution from $T_{1}$ is

$$
\begin{aligned}
& T_{1}=\frac{1}{2} \sum_{\substack{\alpha \in \Delta_{\mu+\epsilon}(\mathfrak{g}(1)) \\
\gamma_{t} \in \Delta_{\mu+\epsilon}(\mathfrak{z}(\mathfrak{n}))}}\left(N_{-\mu, \gamma_{t}-\epsilon} N_{-\epsilon, \gamma_{t}}\right)\left(N_{\alpha,-\gamma_{t}} N_{-\theta\left(\gamma_{t}\right), \theta(\alpha)}\right) R\left(\left[\left[X_{\mu}, X_{-\alpha}\right], X_{-\theta(\alpha)}\right]\right)_{e} \\
& =\frac{1}{2} \sum_{\substack{\alpha \in \Delta_{\mu+\epsilon}(\mathfrak{g}(1)) \backslash\{\mu, \epsilon\} \\
\gamma_{t} \in \Delta_{\mu+\epsilon}(\mathfrak{z}(\mathfrak{n}))}}\left(N_{-\mu, \gamma_{t}-\epsilon} N_{-\epsilon, \gamma_{t}}\right)\left(N_{\alpha,-\gamma_{t}} N_{-\theta\left(\gamma_{t}\right), \theta(\alpha)}\right) \\
& \times R\left(\left[\left[X_{\mu}, X_{-\alpha}\right], X_{-\theta(\alpha)}\right]\right)_{e} \\
& =\frac{1}{2} \sum_{\substack{\alpha \in \Delta_{\mu+\epsilon}(\mathfrak{g}(1)) \backslash\{\mu, \epsilon\} \\
\gamma_{t} \in \Delta_{\mu+\epsilon}(\mathfrak{z}(\mathfrak{n}))}}\left(N_{-\mu, \gamma_{t}-\epsilon} N_{-\epsilon, \gamma_{t}}\right)\left(N_{\alpha,-\gamma_{t}} N_{-\theta\left(\gamma_{t}\right), \theta(\alpha)}\right) \\
& \times\left(N_{\mu,-\alpha} N_{\mu-\alpha,-\theta(\alpha)}\right) R\left(X_{-\epsilon}\right)_{e} \\
& =\frac{1}{2} \sum_{\substack{\alpha \in \Delta_{\mu+\epsilon}(\mathfrak{g}(1)) \backslash\{\mu, \epsilon\} \\
\gamma_{t} \in \Delta_{\mu+\epsilon}(\mathfrak{z}(\mathfrak{n}))}}\left(N_{-\mu, \gamma_{t}-\epsilon} N_{-\epsilon, \gamma_{t}}\right)\left(N_{\alpha,-\gamma_{t}} N_{-\theta\left(\gamma_{t}\right), \theta(\alpha)}\right) \\
& \times\left(N_{\mu,-\alpha} N_{-\theta(\alpha), \theta(\mu)}\right) R\left(X_{-\epsilon}\right)_{e} \\
& =\frac{1}{2} \sum_{\substack{\alpha \in \Delta_{\mu+\epsilon}(\mathfrak{g}(1)) \backslash\{\mu, \epsilon\} \\
\gamma_{t} \in \Delta_{\mu+\epsilon}(\mathfrak{z}(\mathfrak{n}))}}\left(N_{-\mu, \gamma_{t}-\epsilon} N_{-\epsilon, \gamma_{t}}\right)\left(N_{\alpha,-\gamma_{t}} N_{\mu,-\alpha} N_{-\theta\left(\gamma_{t}\right), \theta(\alpha)} N_{-\theta(\alpha), \theta(\mu)}\right) \\
& \times R\left(X_{-\epsilon}\right) \text {. }
\end{aligned}
$$

Note that, from the third equality to the fourth, we use that $N_{\mu-\alpha,-\theta(\alpha)}=N_{-\theta(\alpha), \theta(\mu)}$, as $(\mu-\alpha)+(-\theta(\alpha))+\theta(\mu)=0$. (See the normalization (H6) in Section 5) By Lemma 6.39, we have

$$
N_{\alpha,-\gamma_{t}} N_{\mu,-\alpha} N_{-\theta\left(\gamma_{t}\right), \theta(\alpha)} N_{-\theta(\alpha), \theta(\mu)}=N_{\mu, \epsilon-\gamma_{t}} N_{\epsilon,-\gamma_{t}} \frac{\left\|\alpha_{\mathfrak{q}}\right\|^{2}}{2} .
$$

Therefore,

$$
\begin{aligned}
T_{1}= & \frac{1}{2} \sum_{\substack{\alpha \in \Delta_{\mu+\epsilon}(\mathfrak{g}(1)) \backslash\{\mu, \epsilon\} \\
\gamma_{t} \in \Delta_{\mu+\epsilon}(\mathfrak{z}(\mathfrak{n}))}}\left(N_{-\mu, \gamma_{t}-\epsilon} N_{-\epsilon, \gamma_{t}}\right)\left(N_{\alpha,-\gamma_{t}} N_{\mu,-\alpha} N_{-\theta\left(\gamma_{t}\right), \theta(\alpha)} N_{-\theta(\alpha), \theta(\mu)}\right) \\
& \times R\left(X_{-\epsilon}\right)_{e} \\
= & \frac{\left\|\alpha_{\mathfrak{q}}\right\|^{2}}{4} \sum_{\substack{\alpha \in \Delta_{\mu+\epsilon}(\mathfrak{g}(1)) \backslash\{\mu, \epsilon\} \\
\gamma_{t} \in \Delta_{\mu+\epsilon}(\mathfrak{z}(\mathfrak{n}))}}\left(N_{-\mu, \gamma_{t}-\epsilon} N_{-\epsilon, \gamma_{t}}\right)\left(N_{\mu, \epsilon-\gamma_{t}} N_{\epsilon,-\gamma_{t}}\right) R\left(X_{-\epsilon}\right)_{e} \\
= & \frac{\left\|\alpha_{\mathfrak{q}}\right\|^{2}}{4} \sum_{\substack{\alpha \in \Delta_{\mu+\epsilon}(\mathfrak{g}(1)) \backslash\{\mu, \epsilon\} \\
\gamma_{t} \in \Delta_{\mu+\epsilon}(\mathfrak{z}(\mathfrak{n}))}}\left(N_{\mu, \epsilon-\gamma_{t}} N_{-\mu, \gamma_{t}-\epsilon} N_{\epsilon,-\gamma_{t}} N_{-\epsilon, \gamma_{t}}\right) R\left(X_{-\epsilon}\right)_{e} \\
= & \frac{\left\|\alpha_{\mathfrak{q}}\right\|^{2}}{4} C(\mu, \epsilon) \sum_{\alpha \in \Delta_{\mu+\epsilon}(\mathfrak{g}(1)) \backslash\{\mu, \epsilon\}} R\left(X_{-\epsilon}\right)_{e} \\
= & \frac{\left\|\alpha_{\mathfrak{q}}\right\|^{2}}{4} C(\mu, \epsilon)\left(\left|\Delta_{\mu+\epsilon}(\mathfrak{g}(1))\right|-2\right) R\left(X_{-\epsilon}\right)_{e} .
\end{aligned}
$$


Hence, we obtain

$$
\begin{aligned}
{\left[\pi_{s}\left(X_{\mu}\right), \Omega_{2}\left(Y_{l}^{*}\right)\right]_{e} } & =T_{1}+T_{2}+T_{3} \\
& =-\frac{\left\|\alpha_{\mathfrak{q}}\right\|^{2}}{2} C(\mu, \epsilon)\left(s-\left(\frac{\left|\Delta_{\mu+\epsilon}(\mathfrak{g}(1))\right|}{2}-1\right)\right) R\left(X_{-\epsilon}\right)_{e} .
\end{aligned}
$$

Now we are going to equation (7.18). If $V(\mu+\epsilon)$ is of type 2 , then $\mu+\epsilon=2 \mu$; in particular, $\theta(\mu)=(2 \mu)-\mu=\mu$. By Lemma 6.28, $\Delta_{2 \mu}(\mathfrak{g}(1))=\{\mu\}$. Thus, (7.10) becomes

$$
\begin{aligned}
\Omega_{2}\left(Y_{l}^{*}\right) & =\frac{1}{2} \sum_{\substack{\alpha \in \Delta_{2 \mu}(\mathfrak{g}(1)) \\
\gamma_{t} \in \Delta_{2 \mu}(\mathfrak{z}(\mathfrak{n}))}}\left(N_{-\mu, \gamma_{t}-\epsilon} N_{-\epsilon, \gamma_{t}}\right)\left(N_{\alpha,-\gamma_{t}} N_{-\theta\left(\gamma_{t}\right), \theta(\alpha)}\right) R\left(X_{-\alpha}\right) R\left(X_{-\theta(\alpha)}\right) \\
& =\frac{1}{2} \sum_{\gamma_{t} \in \Delta_{2 \mu}(\mathfrak{z}(\mathfrak{n}))}\left(N_{-\mu, \gamma_{t}-\mu} N_{-\mu, \gamma_{t}}\right)\left(N_{\mu,-\gamma_{t}} N_{-\theta\left(\gamma_{t}\right), \theta(\mu)}\right) R\left(X_{-\mu}\right) R\left(X_{-\theta(\mu)}\right) \\
(7.21) & =\frac{1}{2} \sum_{\gamma_{t} \in \Delta_{2 \mu}(\mathfrak{z}(\mathfrak{n}))}\left(N_{-\mu, \gamma_{t}-\mu} N_{-\mu, \gamma_{t}}\right)\left(N_{\mu,-\gamma_{t}} N_{-\theta\left(\gamma_{t}\right), \mu}\right) R\left(X_{-\mu}\right)^{2} .
\end{aligned}
$$

Since $\left(-\theta\left(\gamma_{t}\right)\right)+\mu+\left(\mu-\gamma_{t}\right)=0$, we have $N_{-\theta\left(\gamma_{t}\right), \mu}=N_{\mu, \mu-\gamma_{t}}$. Thus,

$$
\begin{aligned}
& \frac{1}{2} \sum_{\gamma_{t} \in \Delta_{2 \mu}(\mathfrak{z}(\mathfrak{n}))}\left(N_{-\mu, \gamma_{t}-\mu} N_{-\mu, \gamma_{t}}\right)\left(N_{\mu,-\gamma_{t}} N_{-\theta\left(\gamma_{t}\right), \mu}\right) R\left(X_{-\mu}\right)^{2} \\
& =\frac{1}{2} \sum_{\gamma_{t} \in \Delta_{2 \mu}(\mathfrak{z}(\mathfrak{n}))}\left(N_{-\mu, \gamma_{t}-\mu} N_{-\mu, \gamma_{t}}\right)\left(N_{\mu,-\gamma_{t}} N_{\mu, \mu-\gamma_{t}}\right) R\left(X_{-\mu}\right)^{2} \\
& =\frac{1}{2} \sum_{\gamma_{t} \in \Delta_{2 \mu}(\mathfrak{z}(\mathfrak{n}))}\left(N_{\mu, \mu-\gamma_{t}} N_{-\mu, \gamma_{t}-\mu} N_{\mu,-\gamma_{t}} N_{-\mu, \gamma_{t}}\right) R\left(X_{-\mu}\right)^{2} \\
& =\frac{1}{2} C(\mu, \mu) R\left(X_{-\mu}\right)^{2} .
\end{aligned}
$$

Therefore,

$$
\left[\pi_{s}\left(X_{\mu}\right), \Omega_{2}\left(Y_{l}^{*}\right)\right]_{e}=\frac{1}{2} C(\mu, \mu)\left[\pi_{s}\left(X_{\mu}\right), R\left(X_{-\mu}\right)^{2}\right]_{e} .
$$

It follows from (7.20) that $\lambda_{\mathfrak{q}}\left(\left[X_{\mu}, X_{-\mu}\right]\right)=\left\|\alpha_{\mathfrak{q}}\right\|^{2} / 2$. Then, by Proposition 7.14 with $\alpha=\beta=\mu$, we have

$$
\begin{aligned}
{\left[\pi_{s}\left(X_{\mu}\right), R\left(X_{-\mu}\right)^{2}\right]_{e} } & =R\left(\left[\left[X_{\mu}, X_{-\mu}\right], X_{-\mu}\right]\right)_{e}-2 s \lambda_{\mathfrak{q}}\left(\left[X_{\mu}, X_{-\mu}\right]\right) R\left(X_{-\mu}\right)_{e} \\
& =-\mu\left(H_{\mu}\right) R\left(X_{-\mu}\right)_{e}-2 s \cdot \frac{\left\|\alpha_{\mathfrak{q}}\right\|^{2}}{2} R\left(X_{-\mu}\right)_{e} \\
& =-\left(s\left\|\alpha_{\mathfrak{q}}\right\|^{2}+\|\mu\|^{2}\right) R\left(X_{-\mu}\right)_{e} .
\end{aligned}
$$

Observe that Table 6 in Subsection 6.2 shows that a special constituent of type 2 occurs only when $\mathfrak{q}$ is of type $B_{n}(n)$, type $C_{n}(i)$ or $F_{4}(4)$. Appendix A shows that when $\mathfrak{q}$ is of these types, we have $\|\mu\|^{2}=\left\|\alpha_{\mathfrak{q}}\right\|^{2}$. Therefore,

$$
\left[\pi_{s}\left(X_{\mu}\right), R\left(X_{-\mu}\right)^{2}\right]_{e}=-\left(s\left\|\alpha_{\mathfrak{q}}\right\|^{2}+\|\mu\|^{2}\right) R\left(X_{-\mu}\right)_{e}=-\left\|\alpha_{\mathfrak{q}}\right\|^{2}(s+1) R\left(X_{-\mu}\right)_{e} .
$$


Hence, we obtain

$$
\begin{aligned}
{\left[\pi_{s}\left(X_{\mu}\right), \Omega_{2}\left(Y_{l}^{*}\right)\right]_{e} } & =\frac{1}{2} C(\mu, \mu)\left[\pi_{s}\left(X_{\mu}\right), R\left(X_{-\mu}\right)^{2}\right]_{e} \\
& =-\frac{\left\|\alpha_{\mathfrak{q}}\right\|^{2}}{2} C(\mu, \mu)(s+1) R\left(X_{-\mu}\right)_{e} .
\end{aligned}
$$

To emphasize the fundamental weight $\lambda_{\mathfrak{q}}$, we write $\mathcal{L}\left(s \lambda_{\mathfrak{q}}\right)$ for the line bundle $\mathcal{L}_{s}$. Now, by combining Proposition 7.13 and Theorem 7.16, we conclude the following.

Corollary 7.23. Under the same hypotheses in Theorem 7.16, we have:

(1) If $V(\mu+\epsilon)^{*}$ is of type $1 a$, then the $\left.\Omega_{2}\right|_{V(\mu+\epsilon)^{*}}$ system is conformally invariant on the line bundle $\mathcal{L}\left(s_{2} \lambda_{\mathfrak{q}}\right)$, where $s_{2}$ is the constant given in Theorem 7.16.

(2) If $V(\mu+\epsilon)^{*}$ is of type 2, then the $\left.\Omega_{2}\right|_{V(\mu+\epsilon) *}$ system is conformally invariant on the line bundle $\mathcal{L}\left(-\lambda_{\mathfrak{q}}\right)$.

Proof. This corollary follows from Proposition 7.13 and Theorem 7.16.

As we defined in Definition 6.19, we denote by $V\left(\mu+\epsilon_{\gamma}\right)$ the special constituent of $\mathfrak{l} \otimes \mathfrak{z}(\mathfrak{n})$ so that $V\left(\mu+\epsilon_{\gamma}\right) \subset \mathfrak{l}_{\gamma} \otimes \mathfrak{z}(\mathfrak{n})$, and denote by $V\left(\mu+\epsilon_{n \gamma}\right)$ the special constituent so that $V\left(\mu+\epsilon_{n \gamma}\right)=\mathfrak{l}_{n \gamma} \otimes \mathfrak{z}(\mathfrak{n})$. See Table 6] in Subsection 6.2 for the types of $V\left(\mu+\epsilon_{\gamma}\right)$ and $V\left(\mu+\epsilon_{n \gamma}\right)$ for each case. Table 7 summarizes the line bundles $\mathcal{L}\left(s_{0} \lambda_{\mathfrak{q}}\right)$ on which the $\Omega_{2}$ systems are conformally invariant. Here, a dash

TABLE 7. Line bundles with special values

\begin{tabular}{c|c|c}
\hline Parabolic subalgebra $\mathfrak{q}$ & $\left.\Omega_{2}\right|_{V\left(\mu+\epsilon_{\gamma}\right)^{*}}$ & $\left.\Omega_{2}\right|_{V\left(\mu+\epsilon_{n \gamma}\right)^{*}}$ \\
\hline$B_{n}(i), 3 \leq i \leq n-2$ & $\mathcal{L}\left(\left(n-i-\frac{1}{2}\right) \lambda_{i}\right)$ & $\mathcal{L}\left(\lambda_{i}\right)$ \\
$B_{n}(n-1)$ & $\mathcal{L}\left(\frac{1}{2} \lambda_{n-1}\right)$ & $?$ \\
$B_{n}(n)$ & $\mathcal{L}\left(-\lambda_{n}\right)$ & - \\
$C_{n}(i), 2 \leq i \leq n-1$ & $?$ & $\mathcal{L}\left(-\lambda_{i}\right)$ \\
$D_{n}(i), 3 \leq i \leq n-3$ & $\mathcal{L}\left((n-i-1) \lambda_{i}\right)$ & $\mathcal{L}\left(\lambda_{i}\right)$ \\
$E_{6}(3)$ & $\mathcal{L}\left(\lambda_{3}\right)$ & $\mathcal{L}\left(2 \lambda_{3}\right)$ \\
$E_{6}(5)$ & $\mathcal{L}\left(\lambda_{5}\right)$ & $\mathcal{L}\left(2 \lambda_{5}\right)$ \\
$E_{7}(2)$ & $\mathcal{L}\left(2 \lambda_{2}\right)$ & - \\
$E_{7}(6)$ & $\mathcal{L}\left(\lambda_{6}\right)$ & $\mathcal{L}\left(3 \lambda_{6}\right)$ \\
$E_{8}(1)$ & $\mathcal{L}\left(3 \lambda_{1}\right)$ & - \\
$F_{4}(4)$ & $\mathcal{L}\left(-\lambda_{4}\right)$ & - \\
\hline
\end{tabular}

indicates that there does not exist the special constituent $V\left(\mu+\epsilon_{n \gamma}\right)$. When $\mathfrak{q}$ is of type $B_{n}(n-1)$, the special constituent $V\left(\mu+\epsilon_{n \gamma}\right)$ is of type $1 \mathrm{~b}$, and when $\mathfrak{q}$ is of type $C_{n}(i)$, the special constituent $V\left(\mu+\epsilon_{\gamma}\right)$ is of type 3 . Therefore, we put a question mark for these cases in the table. We close these cases in 22] together with the case when $\mathfrak{q}$ is of type $D_{n}(n-2)$. 


\section{Appendix A. Miscellaneous Data}

This appendix summarizes the miscellenious data for the maximal parabolic subalgebras $\mathfrak{q}=\mathfrak{l} \oplus \mathfrak{g}(1) \oplus \mathfrak{z}(\mathfrak{n})$ of quasi-Heisenberg type shown in (4.9) and (4.10) in Section 4. For each case we give the deleted Dynkin diagram of $\mathfrak{q}$, the subgraphs for $\mathfrak{l}_{\gamma}$ and $\mathfrak{l}_{n \gamma}$, the simple root $\alpha_{\gamma}$ that is not orthogonal to the highest root for $\mathfrak{g}$, the highest weights for $\mathfrak{g}(1)$ and $\mathfrak{z}(\mathfrak{n})$, and the highest roots for $\mathfrak{l}_{\gamma}$ and $\mathfrak{l}_{n \gamma}$. For the definition for the deleted Dynkin diagram see Subsection 4.1. Subsection 4.2 describes the subspaces $\mathfrak{g}(1)$ and $\mathfrak{z}(\mathfrak{n})$. The definitions for the simple ideals $\mathfrak{l}_{\gamma}$ and $\mathfrak{l}_{n \gamma}$ of $\mathfrak{l}$ are given in Subsection 4.3. For classical algebras the sets of roots contributing to $\mathfrak{g}(1), \mathfrak{z}(\mathfrak{n}), \mathfrak{l}_{\gamma}$, and $\mathfrak{l}_{n \gamma}$ are given in the standard realization of the roots.

$$
\S \mathrm{B}_{n}(i), 3 \leq i \leq n-2
$$

(1) The deleted Dynkin diagram:

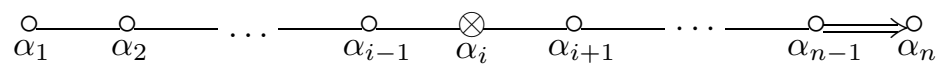

(2) The subgraph for $\mathfrak{l}_{\gamma}$ :

(3) The subgraph for $\mathfrak{l}_{n \gamma}$ :
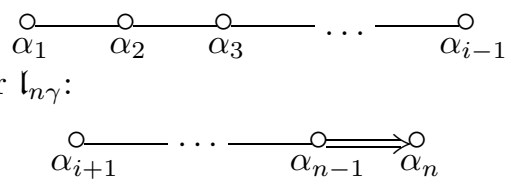

We have $\alpha_{\gamma}=\alpha_{2}$. The highest weight $\mu$ and the set of roots $\Delta(\mathfrak{g}(1))$ for $\mathfrak{g}(1)$ are $\mu=\varepsilon_{1}+\varepsilon_{i+1}$ and $\Delta(\mathfrak{g}(1))=\left\{\varepsilon_{j} \pm \varepsilon_{k} \mid 1 \leq j \leq i\right.$ and $\left.i+1 \leq k \leq n\right\} \cup\left\{\varepsilon_{j} \mid 1 \leq j \leq\right.$ $i\}$. The highest weight $\gamma$ and the set of roots $\Delta(\mathfrak{z}(\mathfrak{n}))$ for $\mathfrak{z}(\mathfrak{n})$ are $\gamma=\varepsilon_{1}+\varepsilon_{2}$ and $\Delta(\mathfrak{z}(\mathfrak{n}))=\left\{\varepsilon_{j}+\varepsilon_{k} \mid 1 \leq j<k \leq i\right\}$. The highest root $\xi_{\gamma}$ and the set of positive roots $\Delta^{+}\left(\mathfrak{l}_{\gamma}\right)$ for $\mathfrak{l}_{\gamma}$ are $\xi_{\gamma}=\varepsilon_{1}-\varepsilon_{i}$ and $\Delta^{+}\left(\mathfrak{l}_{\gamma}\right)=\left\{\varepsilon_{j}-\varepsilon_{k} \mid 1 \leq j<k \leq i\right\}$. The highest root $\xi_{n \gamma}$ and the set of positive roots $\Delta^{+}\left(\mathfrak{l}_{n \gamma}\right)$ for $\mathfrak{l}_{n \gamma}$ are $\xi_{n \gamma}=\varepsilon_{i+1}+\varepsilon_{i+2}$ and $\Delta^{+}\left(\mathfrak{l}_{n \gamma}\right)=\left\{\varepsilon_{j} \pm \varepsilon_{k} \mid i+1 \leq j<k \leq n\right\} \cup\left\{\varepsilon_{j} \mid i+1 \leq j \leq n\right\}$.

$$
\S \mathrm{B}_{n}(n-1)
$$

(1) The deleted Dynkin diagram:

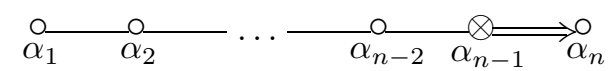

(2) The subgraph for $\mathfrak{l}_{\gamma}$ :

(3) The subgraph for $\mathfrak{l}_{n \gamma}$ :

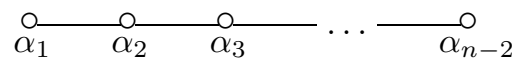

$$
\stackrel{\circ}{\alpha_{n}}
$$

We have $\alpha_{\gamma}=\alpha_{2}$. The highest weight $\mu$ and the set of weights $\Delta(\mathfrak{g}(1))$ for $\mathfrak{g}(1)$ are $\mu=\varepsilon_{1}+\varepsilon_{n}$ and $\Delta(\mathfrak{g}(1))=\left\{\varepsilon_{j} \pm \varepsilon_{n} \mid 1 \leq j \leq n-1\right\} \cup\left\{\varepsilon_{j} \mid 1 \leq j \leq n-1\right\}$. The highest weight $\gamma$ and the set of weights $\mathfrak{g}(\mathfrak{z}(\mathfrak{n}))$ for $\mathfrak{z}(\mathfrak{n}))$ are $\gamma=\varepsilon_{1}+\varepsilon_{2}$ and $\Delta(\mathfrak{z}(\mathfrak{n}))=\left\{\varepsilon_{j}+\varepsilon_{k} \mid 1 \leq j<k \leq n-1\right\}$. The highest root $\xi_{\gamma}$ and the set of positive roots $\Delta^{+}\left(\mathfrak{l}_{\gamma}\right)$ for $\mathfrak{l}_{\gamma}$ are $\xi_{\gamma}=\varepsilon_{1}-\varepsilon_{n-1}$ and $\Delta^{+}\left(\mathfrak{l}_{\gamma}\right)=\left\{\varepsilon_{j}-\varepsilon_{k} \mid 1 \leq j<k \leq n-1\right\}$. The highest root $\xi_{n \gamma}$ and the set of positive roots $\Delta^{+}\left(\mathfrak{l}_{n \gamma}\right)$ for $\mathfrak{l}_{n \gamma}$ are $\xi_{n \gamma}=\varepsilon_{n}$ and $\Delta^{+}\left(\mathfrak{l}_{n \gamma}\right)=\left\{\varepsilon_{n}\right\}$.

$$
\S \mathrm{B}_{n}(n)
$$


(1) The deleted Dynkin diagram:

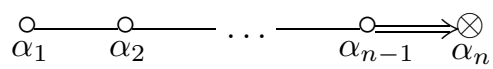

(2) The subgraph for $\mathfrak{l}_{\gamma}$ :

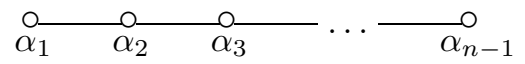

(3) No subgraph for $\mathfrak{l}_{n \gamma}\left(\mathfrak{l}_{n \gamma}=\{0\}\right)$.

We have $\alpha_{\gamma}=\alpha_{2}$. The highest weight $\mu$ and the set of weights $\Delta(\mathfrak{g}(1))$ are $\mu=\varepsilon_{1}$ and $\Delta(\mathfrak{g}(1))=\left\{\varepsilon_{j} \mid 1 \leq j \leq n\right\}$. The highest weight $\gamma$ and the set of weights $\Delta(\mathfrak{z}(\mathfrak{n}))$ for $\mathfrak{z}(\mathfrak{n})$ are $\gamma=\varepsilon_{1}+\varepsilon_{2}$ and $\Delta(\mathfrak{z}(\mathfrak{n}))=\left\{\varepsilon_{j}+\varepsilon_{k} \mid 1 \leq j<k \leq n\right\}$. The highest root $\xi_{\gamma}$ and the set of positive roots for $\mathfrak{l}_{\gamma}$ are $\xi_{\gamma}=\varepsilon_{1}-\varepsilon_{n}$ and $\Delta^{+}\left(\mathfrak{l}_{\gamma}\right)=\left\{\varepsilon_{j}-\varepsilon_{k} \mid 1 \leq j<k \leq n\right\}$.

$$
\S \mathrm{C}_{n}(i), 2 \leq i \leq n-1
$$

(1) The deleted Dynkin diagram:

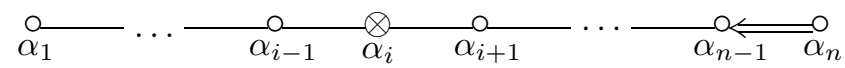

(2) The subgraph for $\mathfrak{l}_{\gamma}$ :

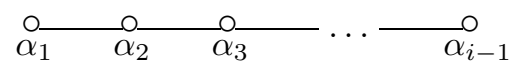

(3) The subgraph for $\mathfrak{l}_{n \gamma}$ :

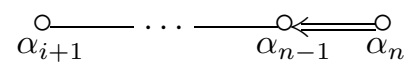

We have $\alpha_{\gamma}=\alpha_{1}$. The highest weight $\mu$ and the set of weights $\Delta(\mathfrak{g}(1))$ for $\mathfrak{g}(1)$ are $\mu=\varepsilon_{1}+\varepsilon_{i+1}$ and $\Delta(\mathfrak{g}(1))=\left\{\varepsilon_{j} \pm \varepsilon_{k} \mid 1 \leq j \leq i\right.$ and $\left.i+1 \leq k \leq n\right\}$. The highest weight $\gamma$ and the set of weights $\Delta(\mathfrak{z}(\mathfrak{n}))$ for $\mathfrak{z}(\mathfrak{n})$ are $\gamma=2 \varepsilon_{1} \Delta(\mathfrak{z}(\mathfrak{n}))=$ $\left\{\varepsilon_{j}+\varepsilon_{k} \mid 1 \leq j<k \leq i\right\} \cup\left\{2 \varepsilon_{j} \mid 1 \leq j \leq i\right\}$. The highest root $\xi_{\gamma}$ and the set of positive roots $\Delta^{+}\left(\mathfrak{l}_{\gamma}\right)$ for $\mathfrak{l}_{\gamma}$ are $\xi_{\gamma}=\varepsilon_{1}-\varepsilon_{i}$ and $\Delta^{+}\left(\mathfrak{l}_{\gamma}\right)=\left\{\varepsilon_{j}-\varepsilon_{k} \mid 1 \leq j<k \leq i\right\}$. The highest root $\xi_{n \gamma}$ and the set of positive roots $\Delta\left(\mathfrak{l}_{n \gamma}\right)$ for $\mathfrak{l}_{n \gamma}$ are $\xi_{n \gamma}=2 \varepsilon_{i+1}$ and $\Delta^{+}\left(\mathfrak{l}_{n \gamma}\right)=\left\{\varepsilon_{j} \pm \varepsilon_{k} \mid i+1 \leq j<k \leq n\right\} \cup\left\{2 \varepsilon_{j} \mid i+1 \leq j \leq n\right\}$.

$$
\S \mathrm{D}_{n}(i), 3 \leq i \leq n-3
$$

(1) The deleted Dynkin diagram:

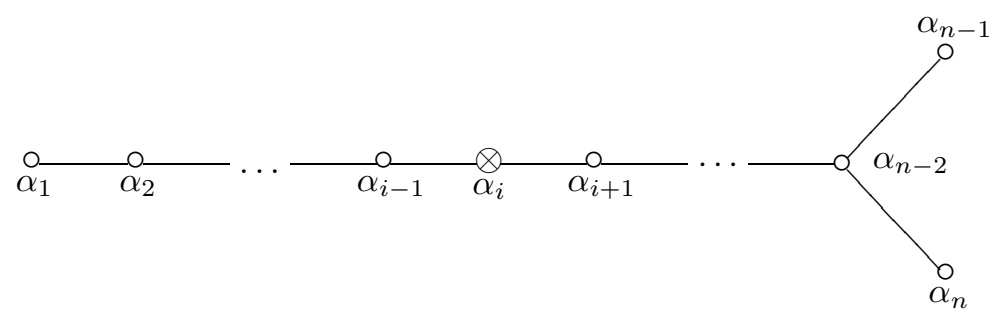

(2) The subgraph for $\mathfrak{l}_{\gamma}$ :

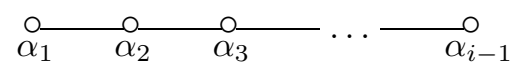


(3) The subgraph for $\mathfrak{l}_{n \gamma}$ :

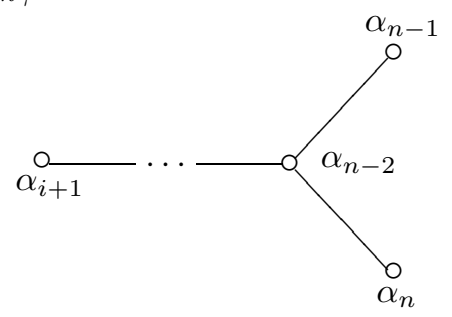

We have $\alpha_{\gamma}=\alpha_{2}$. The highest weight $\mu$ and the set of weights $\Delta(\mathfrak{g}(1))$ for $\mathfrak{g}(1)$ are $\mu=\varepsilon_{1}+\varepsilon_{i+1}$ and $\Delta(\mathfrak{g}(1))=\left\{\varepsilon_{j} \pm \varepsilon_{k} \mid 1 \leq j \leq i\right.$ and $\left.i+1 \leq k \leq n\right\}$. The highest weight $\gamma$ and the set of weights $\Delta(\mathfrak{z}(\mathfrak{n}))$ for $\mathfrak{z}(\mathfrak{n}))$ are $\gamma=\varepsilon_{1}+\varepsilon_{2}$ and $\Delta(\mathfrak{z}(\mathfrak{n}))=\left\{\varepsilon_{j}+\varepsilon_{k} \mid 1 \leq j<k \leq i\right\}$. The highest root $\xi_{\gamma}$ and the set of positive roots $\Delta^{+}\left(\mathfrak{l}_{\gamma}\right)$ for $\mathfrak{l}_{\gamma}$ are $\xi_{\gamma}=\varepsilon_{1}-\varepsilon_{i}$ and $\Delta^{+}\left(\mathfrak{l}_{\gamma}\right)=\left\{\varepsilon_{j}-\varepsilon_{k} \mid 1 \leq j<k \leq i\right\}$. The highest root $\xi_{n \gamma}$ and the set of positive roots $\Delta^{+}\left(\mathfrak{l}_{n \gamma}\right)$ for $\mathfrak{l}_{n \gamma}$ are $\xi_{n \gamma}=\varepsilon_{i+1}+\varepsilon_{i+2}$ $\Delta^{+}\left(\mathfrak{l}_{n \gamma}\right)=\left\{\varepsilon_{j} \pm \varepsilon_{k} \mid i+1 \leq j<k \leq n\right\}$.

$$
\S \mathrm{E}_{6}(3)
$$

(1) The deleted Dynkin diagram:

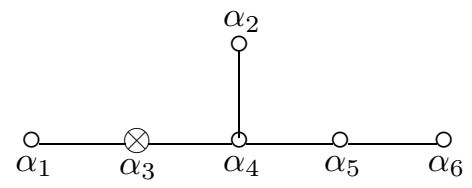

(2) The subgraph for $\mathfrak{l}_{\gamma}$ :

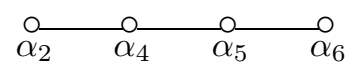

(3) The subgraph for $\mathfrak{l}_{n \gamma}$ :

$$
\stackrel{\circ}{\alpha_{1}}
$$

We have $\alpha_{\gamma}=\alpha_{2}$. The highest weight $\mu$ for $\mathfrak{g}(1)$ is $\mu=\alpha_{1}+\alpha_{2}+\alpha_{3}+2 \alpha_{4}+$ $2 \alpha_{5}+\alpha_{6}$. The highest weight $\gamma$ for $\mathfrak{z}(\mathfrak{n})$ is $\gamma=\alpha_{1}+2 \alpha_{2}+2 \alpha_{3}+3 \alpha_{4}+2 \alpha_{5}+\alpha_{6}$. The highest root $\xi_{\gamma}$ for $\mathfrak{l}_{\gamma}$ is $\xi_{\gamma}=\alpha_{2}+\alpha_{4}+\alpha_{5}+\alpha_{6}$. The highest root $\xi_{n \gamma}$ for $\mathfrak{l}_{n \gamma}$ is $\xi_{n \gamma}=\alpha_{1}$.

$$
\S \mathrm{E}_{6}(5)
$$

(1) The deleted Dynkin diagram:

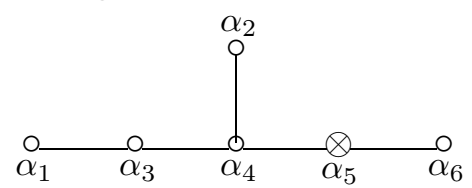

(2) The subgraph for $\mathfrak{l}_{\gamma}$ :

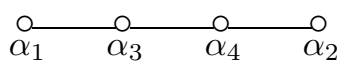

(3) The subgraph for $\mathfrak{l}_{n \gamma}$ :

$$
\stackrel{\circ}{\alpha_{6}}
$$


We have $\alpha_{\gamma}=\alpha_{2}$. The highest weight $\mu$ for $\mathfrak{g}(1)$ is $\mu=\alpha_{1}+\alpha_{2}+2 \alpha_{3}+2 \alpha_{4}+$ $\alpha_{5}+\alpha_{6}$. The highest weight $\gamma$ for $\mathfrak{z}(\mathfrak{n})$ is $\gamma=\alpha_{1}+2 \alpha_{2}+2 \alpha_{3}+3 \alpha_{4}+2 \alpha_{5}+\alpha_{6}$. The highest weight $\xi_{\gamma}$ for $\mathfrak{l}_{\gamma}$ is $\xi_{\gamma}=\alpha_{1}+\alpha_{2}+\alpha_{3}+\alpha_{4}$. The highest weight $\xi_{n \gamma}$ for $\mathfrak{l}_{n \gamma}$ is $\xi_{n \gamma}=\alpha_{6}$.

$$
\S \mathrm{E}_{7}(2)
$$

(1) The deleted Dynkin diagram:

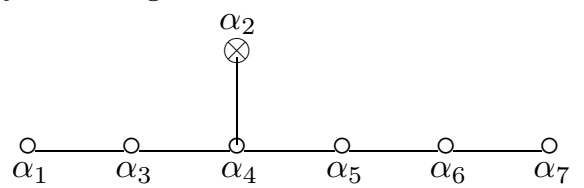

(2) The subgraph for $\mathfrak{l}_{\gamma}$ :

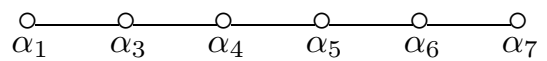

(3) No subgraph for $\mathfrak{l}_{n \gamma}\left(\mathfrak{l}_{n \gamma}=\{0\}\right)$.

We have $\alpha_{\gamma}=\alpha_{1}$. The highest weight $\mu$ for $\mathfrak{g}(1)$ is $\mu=\alpha_{1}+\alpha_{2}+2 \alpha_{3}+3 \alpha_{4}+3 \alpha_{5}+$ $2 \alpha_{6}+\alpha_{7}$. The highest weight $\gamma$ for $\mathfrak{z}(\mathfrak{n})$ is $\gamma=2 \alpha_{1}+2 \alpha_{2}+3 \alpha_{3}+4 \alpha_{4}+3 \alpha_{5}+2 \alpha_{6}+\alpha_{7}$. The highest root $\xi_{\gamma}$ for $\mathfrak{l}_{\gamma}$ is $\xi_{\gamma}=\alpha_{1}+\alpha_{3}+\alpha_{4}+\alpha_{5}+\alpha_{6}+\alpha_{7}$.

$$
\S \mathrm{E}_{7}(6)
$$

(1) The deleted Dynkin diagram:

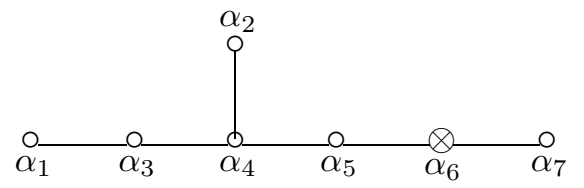

(2) The subgraph for $\mathfrak{l}_{\gamma}$ :

(3) The subgraph for $\mathfrak{l}_{n \gamma}$ :

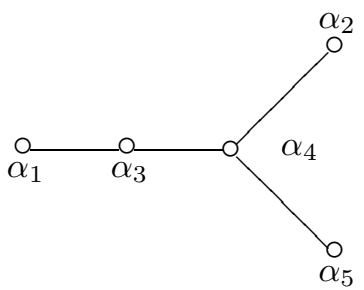

$$
\stackrel{\circ}{\alpha_{7}}
$$

We have $\alpha_{\gamma}=\alpha_{1}$. The highest weight $\mu$ for $\mathfrak{g}(1)$ is $\mu=\alpha_{1}+2 \alpha_{2}+2 \alpha_{3}+3 \alpha_{4}+$ $2 \alpha_{5}+\alpha_{6}+\alpha_{7}$. The highest weight $\gamma$ for $\mathfrak{z}(\mathfrak{n})$ is $\gamma=2 \alpha_{1}+2 \alpha_{2}+3 \alpha_{3}+4 \alpha_{4}+3 \alpha_{5}+$ $2 \alpha_{6}+\alpha_{7}$. The highest root $\xi_{\gamma}$ for $\mathfrak{l}_{\gamma}$ is $\xi_{\gamma}=\alpha_{1}+\alpha_{2}+2 \alpha_{3}+2 \alpha_{4}+\alpha_{5}$. The highest root $\xi_{n \gamma}$ for $\mathfrak{l}_{n \gamma}$ is $\xi_{n \gamma}=\alpha_{7}$.

$$
\S \mathrm{E}_{8}(1)
$$

(1) The deleted Dynkin diagram:

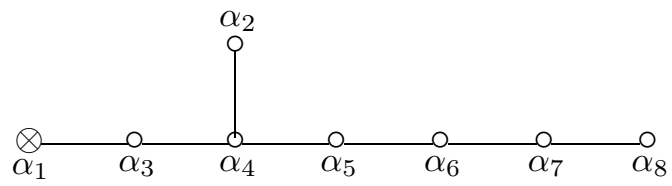


(2) The subgraph for $\mathfrak{l}_{\gamma}$ :

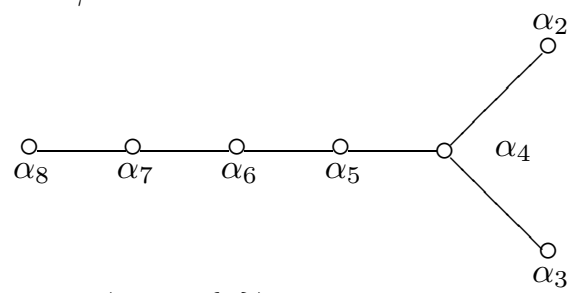

(3) No subgraph for $\mathfrak{l}_{n \gamma}\left(\mathfrak{l}_{n \gamma}=\{0\}\right)$.

We have $\alpha_{\gamma}=\alpha_{8}$. The highest weight $\mu$ for $\mathfrak{g}(1)$ is $\mu=\alpha_{1}+3 \alpha_{2}+3 \alpha_{3}+5 \alpha_{4}+$ $4 \alpha_{5}+3 \alpha_{6}+2 \alpha_{7}+\alpha_{8}$. The highest weight $\gamma$ for $\mathfrak{z}(\mathfrak{n})$ is $\gamma=2 \alpha_{1}+3 \alpha_{2}+4 \alpha_{3}+6 \alpha_{4}+5 \alpha_{5}+$ $4 \alpha_{6}+3 \alpha_{7}+2 \alpha_{8}$. The highest root $\xi_{\gamma}$ for $\mathfrak{l}_{\gamma}$ is $\xi_{\gamma}=\alpha_{2}+\alpha_{3}+2 \alpha_{4}+2 \alpha_{5}+2 \alpha_{6}+2 \alpha_{7}+\alpha_{8}$.

$$
\S \mathrm{F}_{4}(4)
$$

(1) The deleted Dynkin diagram:

(2) The subgraph for $\mathfrak{l}_{\gamma}$ :

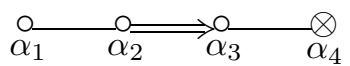

(3) No subgraph for $\mathfrak{l}_{n \gamma}\left(\mathfrak{l}_{n \gamma}=\{0\}\right)$.

We have $\alpha_{\gamma}=\alpha_{1}$. The highest weight $\mu$ for $\mathfrak{g}(1)$ is $\mu=\alpha_{1}+2 \alpha_{2}+3 \alpha_{3}+\alpha_{4}$. The highest weight $\gamma$ for $\mathfrak{z}(\mathfrak{n})$ is $\gamma=2 \alpha_{1}+3 \alpha_{2}+4 \alpha_{3}+2 \alpha_{4}$. The highest root $\xi_{\gamma}$ for $\mathfrak{l}_{\gamma}$ is $\xi_{\gamma}=\alpha_{1}+2 \alpha_{2}+2 \alpha_{3}$.

\section{ACKNOWLEDGMENTS}

This work is part of the author's Ph.D. thesis at Oklahoma State University. The author would like to thank his advisor, Leticia Barchini, for her generous guidance. He would also like to thank Anthony Kable and Roger Zierau for their valuable comments on this work. Finally, the author is thankful to the referee for the careful reading.

\section{REFERENCES}

[1] L. Barchini, Anthony C. Kable, and Roger Zierau, Conformally invariant systems of differential equations and prehomogeneous vector spaces of Heisenberg parabolic type, Publ. Res. Inst. Math. Sci. 44 (2008), no. 3, 749-835. MR2451611 (2009k:22021)

[2] L. Barchini, Anthony C. Kable, and Roger Zierau, Conformally invariant systems of differential operators, Adv. Math. 221 (2009), no. 3, 788-811, DOI 10.1016/j.aim.2009.01.006. MR2511038 (2010d:22020)

[3] Nicolas Bourbaki, Éléments de mathématique (French), Masson, Paris, 1981. Groupes et algèbres de Lie. Chapitres 4, 5 et 6. [Lie groups and Lie algebras. Chapters 4, 5 and 6]. MR647314 (83g:17001)

[4] Mark G. Davidson, Thomas J. Enright, and Ronald J. Stanke, Differential operators and highest weight representations, Mem. Amer. Math. Soc. 94 (1991), no. 455, iv+102, DOI 10.1090/memo/0455. MR:1081660 (92c:22034)

[5] V.K. Dobrev, Invariant differential operators for non-compact Lie algebras parabolically related to conformal Lie algebras, [arXiv:1208.0409].

[6] _ Canonical construction of differential operators intertwining representations of real semisimple Lie groups, Rep. Math. Phys. 25 (1988), no. 2, 159-181, first as ICTP Trieste preprint IC/86/393 (1986).

[7] Leon Ehrenpreis, Hypergeometric functions, Algebraic analysis, Vol. I, Academic Press, Boston, MA, 1988, pp. 85-128. MR992449 (90f:33004) 
[8] S. Helgason, Differential geometry, Lie groups, and symmetric spaces, Academic Press, New York, 1978, reprinted with corrections: American Mathematical Society, Providence, RI, 2001.

[9] Jing-Song Huang, Intertwining differential operators and reducibility of generalized Verma modules, Math. Ann. 297 (1993), no. 2, 309-324, DOI 10.1007/BF01459504. MR1241809 (94k:22034)

[10] James E. Humphreys, Introduction to Lie algebras and representation theory, SpringerVerlag, New York, 1972. Graduate Texts in Mathematics, Vol. 9. MR0323842 (48 \#2197)

[11] Anthony C. Kable, K-finite solutions to conformally invariant systems of differential equations, Tohoku Math. J. (2) 63 (2011), no. 4, 539-559, DOI 10.2748/tmj/1325886280. MR2872955(2012k:22022)

[12] Anthony C. Kable, Conformally invariant systems of differential equations on flag manifolds for $G_{2}$ and their K-finite solutions, J. Lie Theory 22 (2012), no. 1, 93-136. MR2859028

[13] Anthony C. Kable, The Heisenberg ultrahyperbolic equation: K-finite and polynomial solutions, Kyoto J. Math. 52 (2012), no. 4, 839-894. MR2998915

[14] Anthony C. Kable, The Heisenberg ultrahyperbolic equation: the basic solutions as distributions, Pacific J. Math. 258 (2012), no. 1, 165-197, DOI 10.2140/pjm.2012.258.165. MR2972482

[15] Anthony C. Kable, On certain conformally invariant systems of differential equations, New York J. Math. 19 (2013), 189-251.

[16] A.U. Klimyk, Decomposition of a tensor product of irreducible representations of a semisimple Lie algebra into a direct sum of irreducible representations, Amer. Math. Soc. Translations 76 (1968). MR0206169(34:5991)

[17] Anthony W. Knapp, Lie groups beyond an introduction, 2nd ed., Progress in Mathematics, vol. 140, Birkhäuser Boston Inc., Boston, MA, 2002. MR.1920389 (2003c:22001)

[18] Toshiyuki Kobayashi and Bent Orsted, Analysis on the minimal representation of $\mathrm{O}(p, q)$. I. Realization via conformal geometry, Adv. Math. 180 (2003), no. 2, 486-512, DOI 10.1016/S0001-8708(03)00012-4. MR2020550(2004k:22018a)

[19] Bertram Kostant, Verma modules and the existence of quasi-invariant differential operators, Non-commutative harmonic analysis (Actes Colloq., Marseille-Luminy, 1974), Springer, Berlin, 1975, pp. 101-128. Lecture Notes in Math., Vol. 466. MR0396853 (53 \#713)

[20] Toshihisa Kubo, Conformally invariant systems of differential operators associated to twostep nilpotent maximal parabolics of non-Heisenberg type, ProQuest LLC, Ann Arbor, MI, 2012. Thesis (Ph.D.)-Oklahoma State University. MR3054871

[21] Toshihisa Kubo, A system of third-order differential operators conformally invariant under $\mathfrak{s l}(3, \mathbb{C})$ and $\mathfrak{s o}(8, \mathbb{C})$, Pacific J. Math. 253 (2011), no. 2, 439-453, DOI 10.2140/pjm.2011.253.439. MR.2878818

[22] Toshihisa Kubo, Systems of differential operators and generalized Verma modules, SIGMA Symmetry Integrability Geom. Methods Appl. 10 (2014), no. 008, 35 pages.

[23] Toshihisa Kubo, On the homomorphisms between the generalized Verma modules arising from conformally invariant systems, J. Lie Theory 23 (2013), no. 3, 847-883.

[24] Toshihisa Kubo, The Dynkin index and conformally invariant systems associated to parabolic subalgebras of Heisenberg type, Osaka J. Math. 51 (2014), no. 2, 359-375. MR3192546

[25] Nolan R. Wallach, The analytic continuation of the discrete series. I, II, Trans. Amer. Math. Soc. 251 (1979), 1-17, 19-37, DOI 10.2307/1998680. MR.531967 (81a:22009)

Graduate School of Mathematical Sciences, The University of Tokyo, 3-8-1 Komaba, MEGURO-KU, TOKYO 153-8914, JAPAN

E-mail address: toskubo@ms.u-tokyo.ac.jp 